\title{
Numerical Investigations on the Influence of Innovative Hub Geometry Design for Augmented Performance in a Low Speed Centrifugal Fan
}

Madhwesh N

Manipal Institute of Technology

K Vasudeva Karanth

Manipal Institute of Technology

N YAGNESH SHARMA ( $\nabla$ yagnesh.sharma@manipal.edu )

Manipal Institute of Technology https://orcid.org/0000-0002-2082-1768

Research article

Keywords: centrifugal fan, ellipsoidal hub, hemi-spherical hub, inlet stall, hub effectiveness

Posted Date: June 4th, 2020

DOl: https://doi.org/10.21203/rs.3.rs-32353/v1

License: (c) This work is licensed under a Creative Commons Attribution 4.0 International License.

Read Full License 


\title{
Numerical Investigations on the Influence of Innovative Hub Geometry Design for Augmented Performance in a Low Speed Centrifugal Fan
}

\author{
Madhwesh $\mathrm{N}^{1}$., K. Vasudeva Karanth ${ }^{1}$ and N. Yagnesh Sharma ${ }^{1}$. \\ ${ }^{1}$ Department of Mechanical and Manufacturing Engineering, Manipal Institute of Technology, \\ Manipal Academy of Higher Education, Manipal - 576104, Karnataka, India \\ *Corresponding author: yagnesh.sharma@manipal.edu \\ Orcid ID: 0000-0002-2082-1768
}

\begin{abstract}
Inlet region of a centrifugal fan is considered as one of the important flow domains which provides air into the impeller with adequate flow incidence. There is a dire need for flow guidance for incoming air in order to minimize induced swirl losses in the vicinity of eye of the impeller. An extrusion type of structure, commonly termed hub, is attached to the impeller of turbo machines which is used to reduce inlet turning losses and thereby enhancing the performance of the machine in terms of overall static pressure rise. It is seen from a careful literature survey that there has not been significant research on the effect of hub of various shapes and sizes on performance improvement. Analytical tool like computational fluid dynamics (CFD) capture the physics of flow losses encountered especially at the inlet region. This research work attempts to explore numerically the contributions of hub of hemi-spherical and ellipsoidal shapes and parametrically varied sizes on overall performance of the fan. The analysis shows that amongst hemi-spherical and ellipsoidal hub configurations considered in this work, an optimized ellipsoidal hub configuration is found to yield a significant contribution of about $8.4 \%$ for head coefficient and $8.6 \%$ in relative theoretical efficiency over the hub-less base configuration. Finally correlations are developed for the optimized hub shape configurations.
\end{abstract}

Keywords: centrifugal fan, ellipsoidal hub, hemi-spherical hub, inlet stall, hub effectiveness 


\section{Introduction}

Generally, the entrance region of the centrifugal impeller is associated with inlet swirl, sympathetic spiral flow and flow stalls near the back shroud surface due to sudden change of flow direction. This causes inlet turning losses and the flow gets disturbed. As a result there is significant amount of energy loss, causing a reduction in the flow characteristics. The loss flow phenomena as well as associated disturbances in the inlet flow domain can be realized effectively using a whole field CFD analysis of the fan. There is a dire need of a suitable geometric intervention at the inlet region of the impeller which can improve the fluid flow behavior thereby augmenting overall performance of the fan. Various researchers have contributed significantly to the effort to streamline the flow characteristics of the turbo machines especially at the entrance region.

Vagnoli S et al. [1] studied the inception of rotating stall in a transonic centrifugal compressor numerically. The effect of inlet distortions caused by a bent pipe installed just ahead of the impeller was studied in detail. They found that suppression of flow distortions takes place for a bent elbow equipped at the inlet of the impeller. Wang $\mathrm{Y}$ et al. [2] performed numerical investigation on centrifugal fan for the comparison purposes of two kinds of inlets: one is standard straight duct and the other is a $90^{\circ}$ curved duct. They showed that the acceleration caused in the straight duct effectively suppresses the inlet flow distortions without hampering the impeller work. Gholamian M et al. [3] conducted numerical study on centrifugal fan with nozzle and diffuser types of inlet configurations. The study revealed that the inlet flow losses are greatly affected by shape of the inlet. The authors concluded that nozzle with lower diameter results in better static pressure recovery of the fan. A CFD study by Son P N et al. [4] on a centrifugal blower was conducted to investigate the significance of bell mouth shapes on the blower output. Parametrically twelve numerical models were developed using various values of bell mouth radii and gaps between the upper fan casing and bell mouth. Frozen rotor method was chosen to analyze transient characteristics of the blower. The turbulence behaviour was captured by wellknown realizable $\mathrm{k}-\varepsilon^{\varepsilon}$ turbulence model. Results revealed that the bell mouth geometry significantly improves the flow coefficient. Yang C et al. [5] performed numerical analysis on a 
centrifugal compressor provided with inlet bleed flow. They found that there is a significant reduction in relative Mach number at the leading edge area of blade tip. The authors concluded that the flow separation is restrained on blade suction side due to the recirculation. Cai [6] performed a comparative study between stator and cantilever hub configurations in a multi stage compressor. He found that there is a marginal improvement in performance for cantilever hub configuration. He reasoned that improvement is because of the fact that the rotating hub counteracts the passage static pressure gradient and alleviates the secondary flow.

It can be discernable from the above review of literature that a whole field flow analysis pertaining to hub treatment in a centrifugal fan has not been the focus of attention so far. The hub has the capability to streamline the air flow thereby improving the overall efficiency of the fan. Hence in this paper, a numerical analysis on the effect of various hub shapes on the overall performance of the centrifugal fan is presented. It also discusses the feasibility of suitable design interventions for the hub in terms of its shape and size.

\section{Numerical Modelling}

\subsection{Geometry details}

The centrifugal fan used for the present study is depicted in Fig 1. It consists of four important flow domains namely a cylindrical inlet, backward swept impeller, a vaned diffuser and a rectangular type volute casing. The geometry of this test rig is based on the technical paper by Meakhail and Park [7]. This configuration is termed hub-less base configuration in this study. 


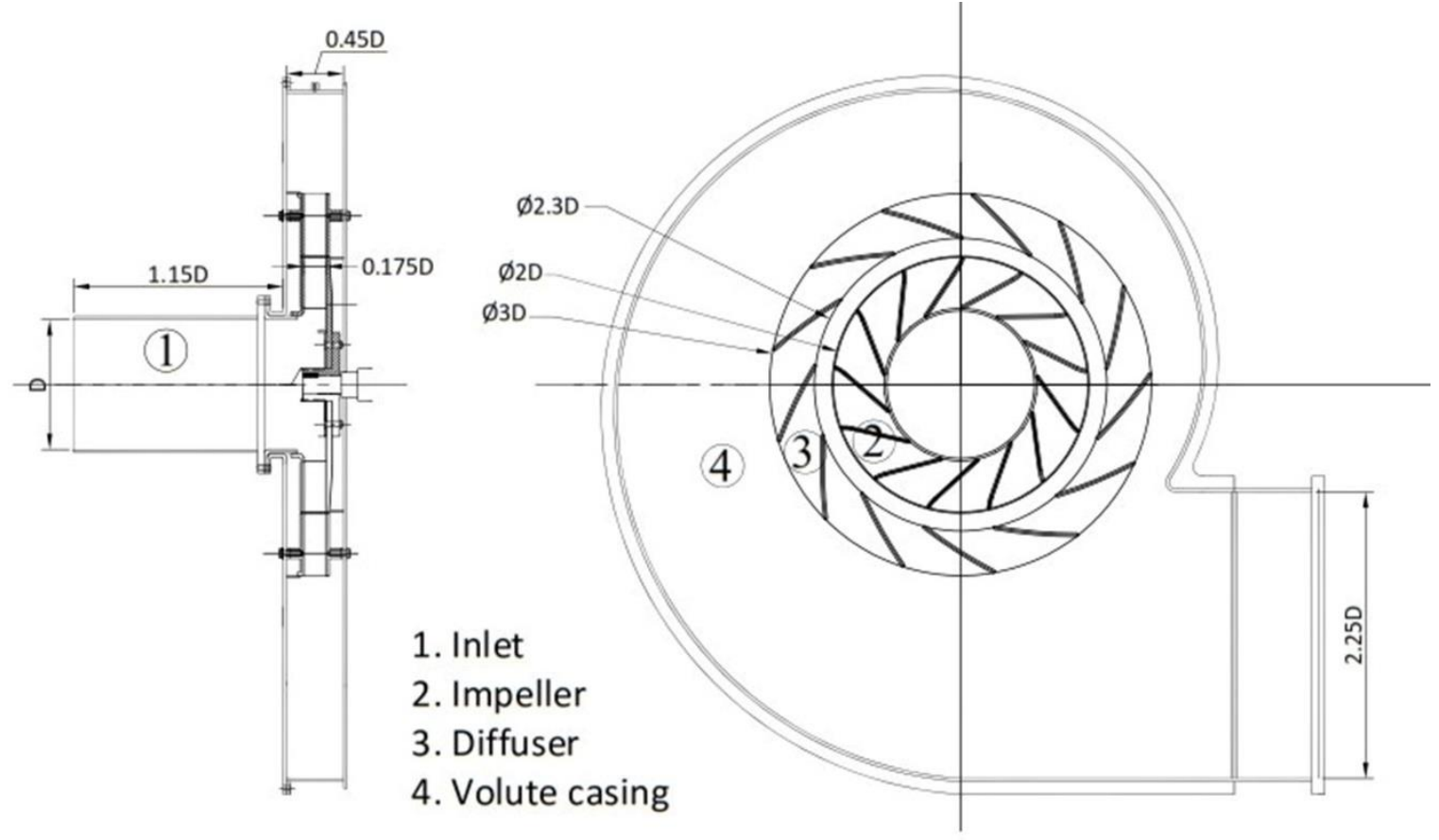

Figure 1: Schematic drawing of centrifugal fan

The cylindrical inlet has an inlet diameter (D) of $200 \mathrm{~mm}$. In order to get conformity in flow properties at the inlet of impeller with that at the fan inlet, an entrance length of about 1.15D is provided. Table 1 illustrates the specifications of the fan.

Table 1: Specifications of hub-less base configuration

\begin{tabular}{ll}
\hline Inlet diameter of impeller & $: 1.2 \mathrm{D}$ \\
Exit diameter of impeller & $: 2 \mathrm{D}$ \\
Inlet diameter of diffuser & $: 2.3 \mathrm{D}$ \\
Exit diameter of diffuser & $: 3 \mathrm{D}$ \\
Thickness of the blades & $: 0.025 \mathrm{D}$ \\
Passage width of impeller and diffuser & $: 0.175 \mathrm{D}$ \\
Radial clearance between impeller and diffuser & $: 0.15 \mathrm{D}$ \\
Channel height of volute casing & $: 0.45 \mathrm{D}$ \\
Volute flange width & $: 2.25 \mathrm{D}$ \\
Blade angle at the inlet of impeller & $: 30^{\circ}$ \\
Blade angle at the exit of impeller & $: 76^{\circ}$
\end{tabular}


Blade angle at the inlet of diffuser $\quad: 23^{\circ}$

Blade angle at the exit of diffuser $\quad: 38^{\circ}$

Number of impeller blades $\quad: 13$

Number of diffuser blades $\quad: 13$

Rated speed of the fan (RPM) $\quad: 1500$

\subsection{Mesh generation and mesh sensitivity analysis}

The exploded view of the computational domain adopted for the present study is illustrated in Fig 2. Sliding mesh technique is adopted for the transient flow analysis as per CFD code used in the present research. This technique requires unstructured mesh domains that are independent of each other connecting but are having overlapping interface zones.

In order to ensure numerical results that are independent of number of control volumes employed for meshing the fluid domain, a mesh sensitivity analysis is carried out. The mesh is generated initially with a coarser mesh corresponding to a total of 4.23 million control volume cells, with a possibility for local refinement of mesh size for the areas of high gradients like the boundary layer wherever required in the domain. The mesh is iteratively augmented with gradual decrement in cell sizes for a finer mesh of the domain corresponding to 9.99 million cells with local mesh refinement. 


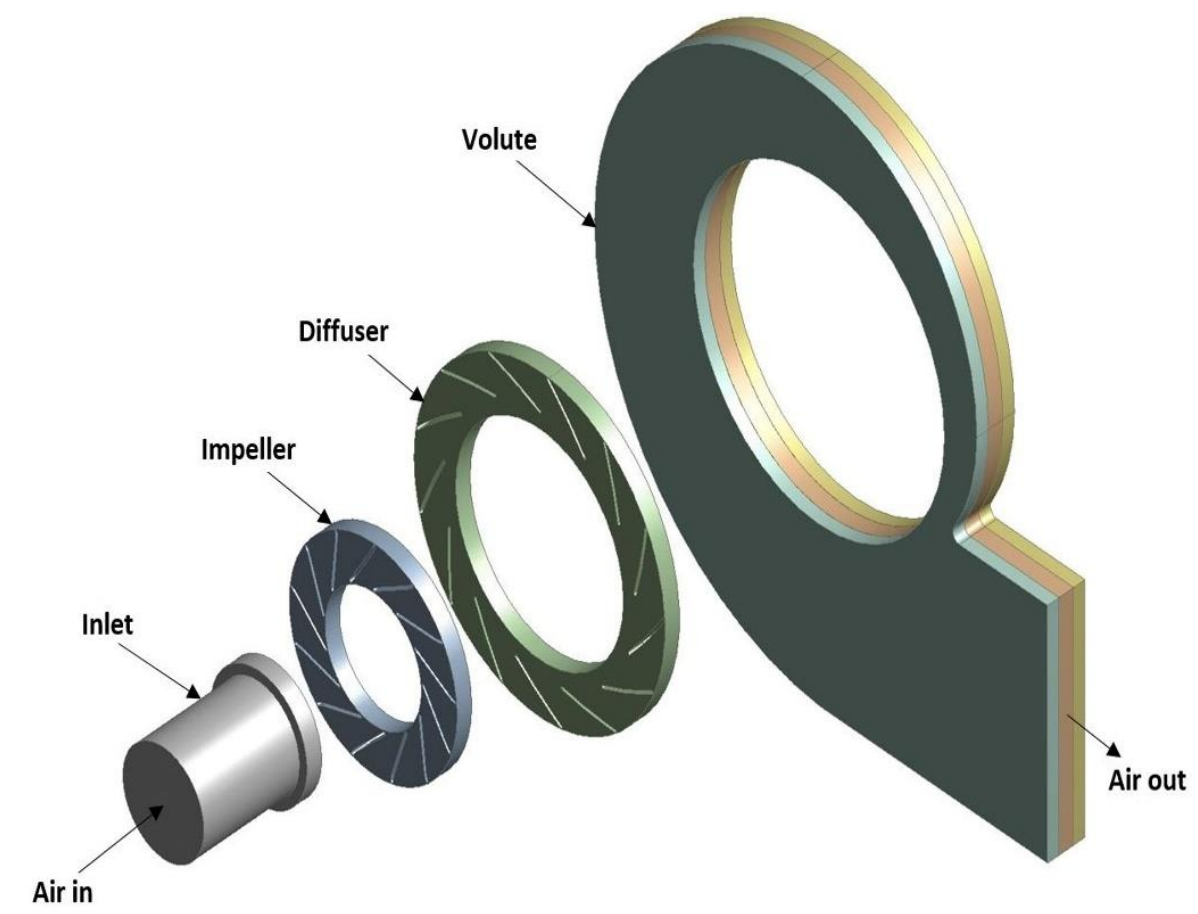

Figure 2: Computational domain of hub-less base configuration considered for the analysis

For the mesh sensitivity study the absolute velocity of air corresponding to the mid-passages of impeller and diffuser domains is adopted as the dependent physical variable for the regions of flow as shown in Fig. 3 for testing the mesh sensitivity for the property chosen. Various runs with coarser to finer control volume mesh densities are performed in the analysis are shown in Fig 4. It is seen that for the mesh density trials corresponding to 6.89 and 9.99 million control volume cells exhibit near convergence of velocity.

Based on the above information, the mesh density with 6.89 million control volume cells is adopted for further numerical analyses. This provides for a minimum memory storage as well as computational time in the present analysis. Fig. 5 depicts a close-up view of meshed domain. Further, the total control volume cells derived from the above study is distributed amongst the flow domains as depicted in Table 2. 


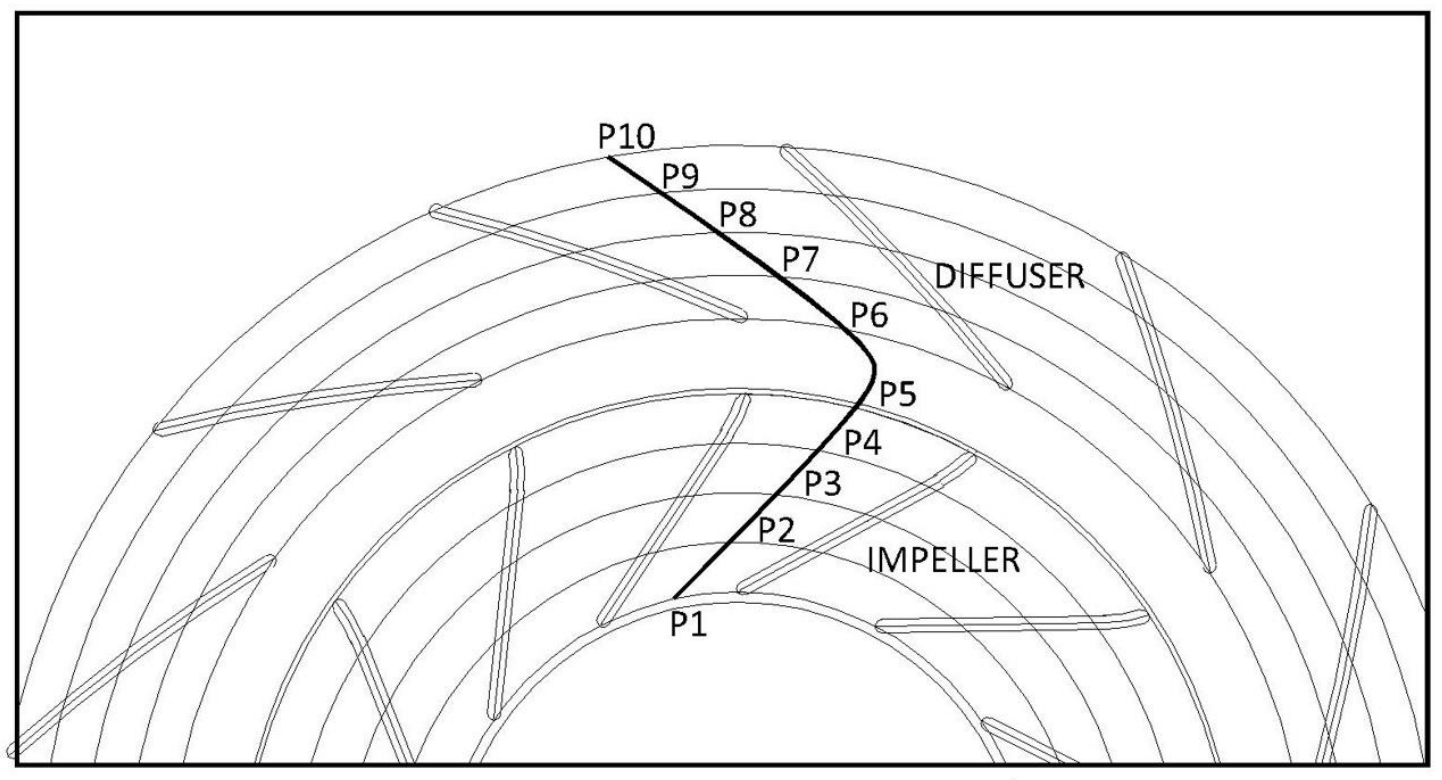

Figure 3: Salient points chosen for mesh sensitivity analysis

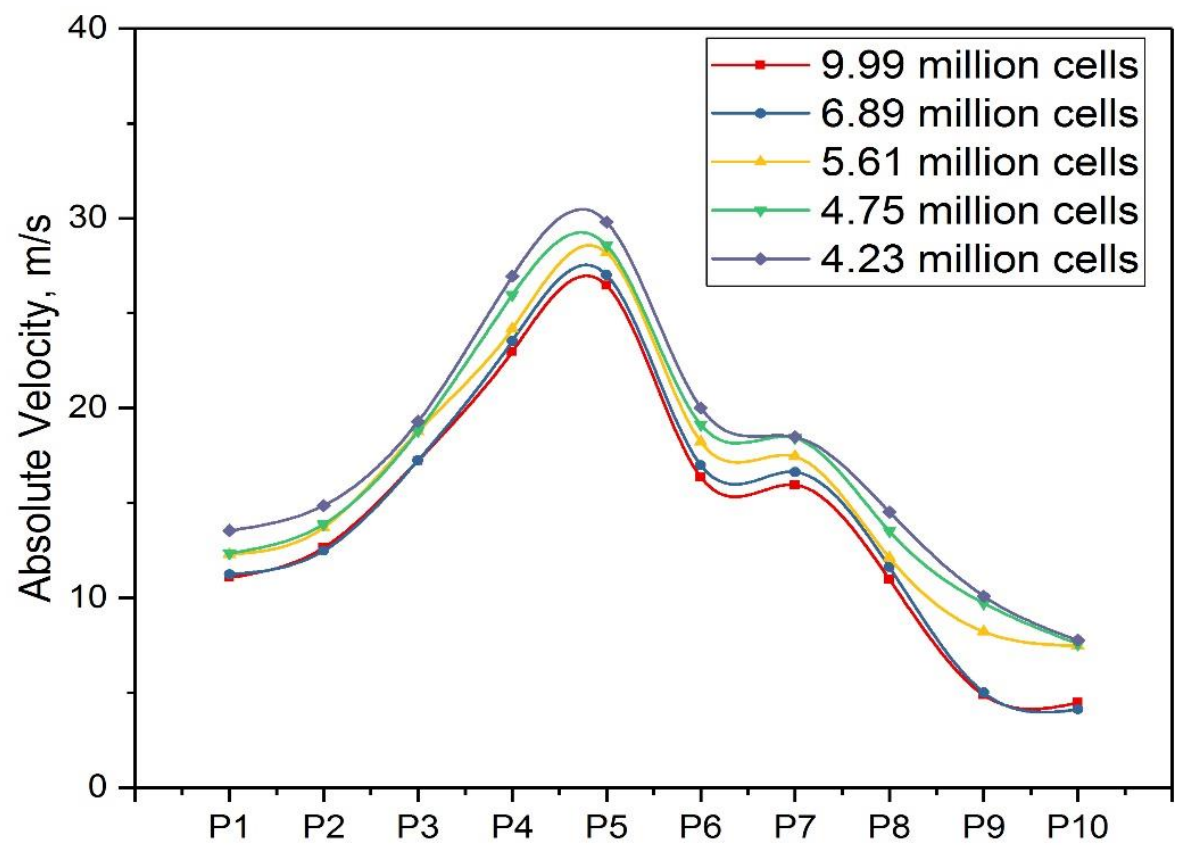

Flow along the Impeller and Diffuser passages

Figure 4: Mesh sensitivity test results based on absolute velocity 


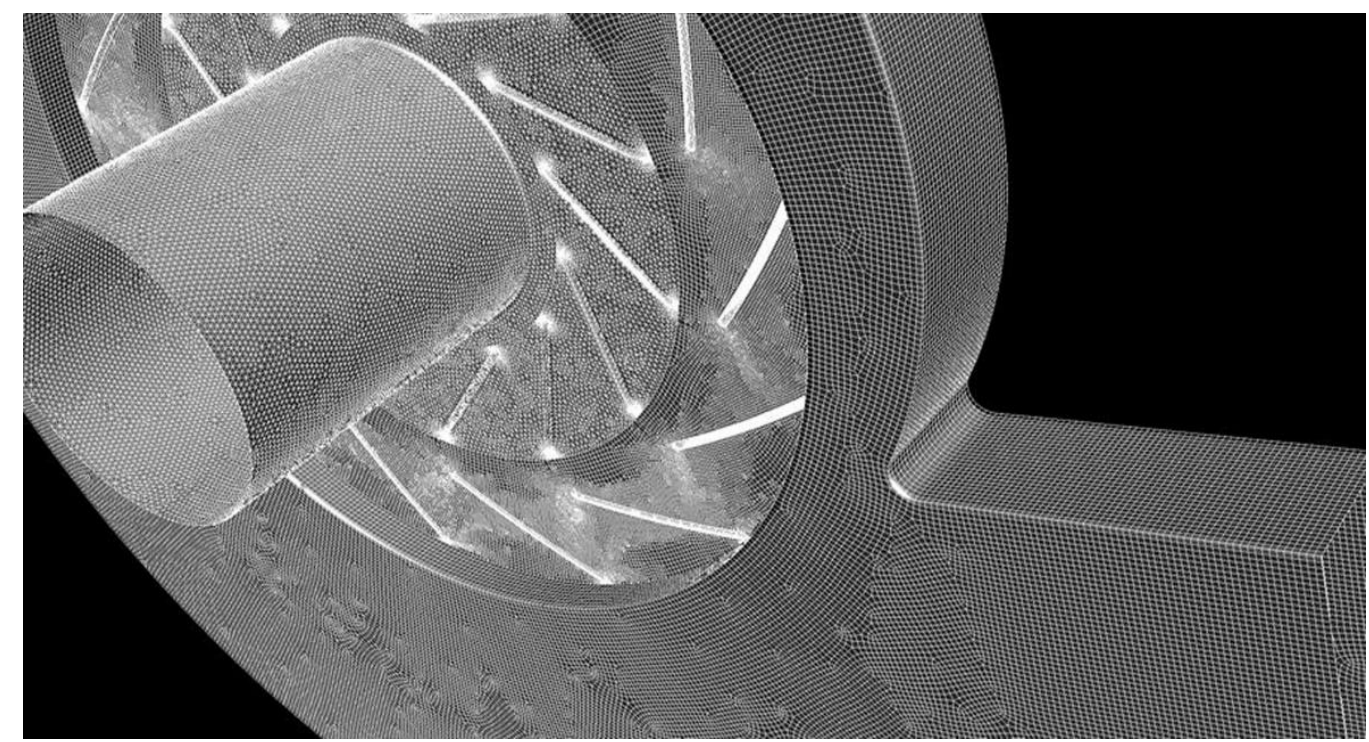

Figure 5: A magnified view of meshed flow regions

Table 2: Distribution of control volume cells in the region of flow

\begin{tabular}{|c|c|c|c|c|}
\hline Regions of flow & Inlet & Impeller & Diffuser & Volute casing \\
\hline $\begin{array}{c}\text { No. of Control } \\
\text { volume cells } \\
\text { (in millions) }\end{array}$ & 1.36 & 1.38 & 2.17 & 1.98 \\
\hline
\end{tabular}

\subsection{Boundary Conditions prescribed for the Numerical Model}

The conservation differential equations for continuity, momentum and scalar transport in the cylindrical frame of reference are solved using well benchmarked CFD code for the sliding mesh domains. The boundary conditions for the numerical analysis are exactly the same as that are available from the associated experimental data generated by the author. This would yield a numerically matching simulation. Corresponding to the bounding surfaces of the domain such as impeller and diffuser blades as well as inner surface of volute casing, a wall boundary condition is imposed with no-slip assumption. It is assumed that the flow is fully developed at the fan exit, hence Neumann boundary condition is imposed along the flow direction. The $k-\varepsilon$ turbulence model is used to capture turbulent flow behaviour with intensity of turbulence set at $5 \%$ as suggested by Meakhail et al [7]. The mean hydraulic diameter required for the analysis is calculated corresponding to fan inlet diameter that would yield the characteristic length as per 
Karanth et al [8]. In the present work an initial set of 100 iterations are carried out for the steady flow to initialize dependent flow variables for the entire domain for the consequent time periodic unsteady flow analysis.

\subsection{Governing Equations}

The governing conservative differential equations for continuity, momentum and scalar transport in the reference frame of the moving mesh may be written in Cartesian tensorial form as given below [9].

Continuity equation:

$$
\frac{d}{d t} \rho+\frac{\partial}{\partial x_{j}} u_{j}-v_{j}=0
$$

Momentum equation:

$$
\frac{d}{d t} \rho_{u_{i}}+\frac{\partial}{\partial x_{j}} \rho u_{j}-v_{j} u_{j}=-\frac{\partial p}{\partial x_{j}}+\frac{\partial}{\partial x_{j}} \tau_{i j}+\tau_{t i j}+S_{u i}
$$

Equation for $\mathrm{k}$ :

$$
\frac{\partial}{\partial_{t}} \rho_{k}+\frac{\partial}{\partial_{x_{j}}} \rho_{u_{i} k}=\frac{\partial}{\partial x_{j}}\left(\frac{\mu}{\sigma_{k}} \frac{\partial_{k}}{\partial x_{j}}\right)+G_{k}-\rho \varepsilon
$$

Equation for $\varepsilon$ :

$$
\frac{\partial}{\partial_{t}} \rho \varepsilon+\frac{\partial}{\partial_{x_{j}}} \rho_{u_{i}} \varepsilon=\frac{\partial}{\partial_{x_{j}}}\left(\frac{\mu}{\sigma_{\varepsilon}} \frac{\partial \varepsilon}{\partial_{x_{j}}}\right)+C_{1^{\varepsilon}} \frac{\varepsilon}{k} G_{k}-C_{2^{\varepsilon}} \rho \frac{\varepsilon^{2}}{k}
$$

\subsection{Calculation Setup for Unsteady Flow Analysis}

The fluid in a centrifugal fan is generally turbulent in nature with prominent flow recirculation. Hence it is required to perform numerical analysis so that the transient flow instabilities are represented as time periodic fluctuations and are captured as the static pressure at various salient regions in the flow domain. In this numerical analysis two full rotations of the impeller are considered for establishment of time periodic state as shown in Fig. 6.

A second order implicit velocity formulation coupled with a pressure based solver is used to perform transient analysis. SIMPLE algorithm of Patankar available in CFD code is employed to 
couple pressure and velocity. Discretization process is governed by second order accurate upwind scheme. Moving mesh methodology is incorporated between the diffuser and the impeller as well as the impeller and the inlet region with a relative movement of one degree rotation between them for each time step of $0.1111 \mathrm{~ms}$. This will essentially fulfil the criteria for stability of the computation scheme for the fan at its rated speed of $1500 \mathrm{rpm}$.

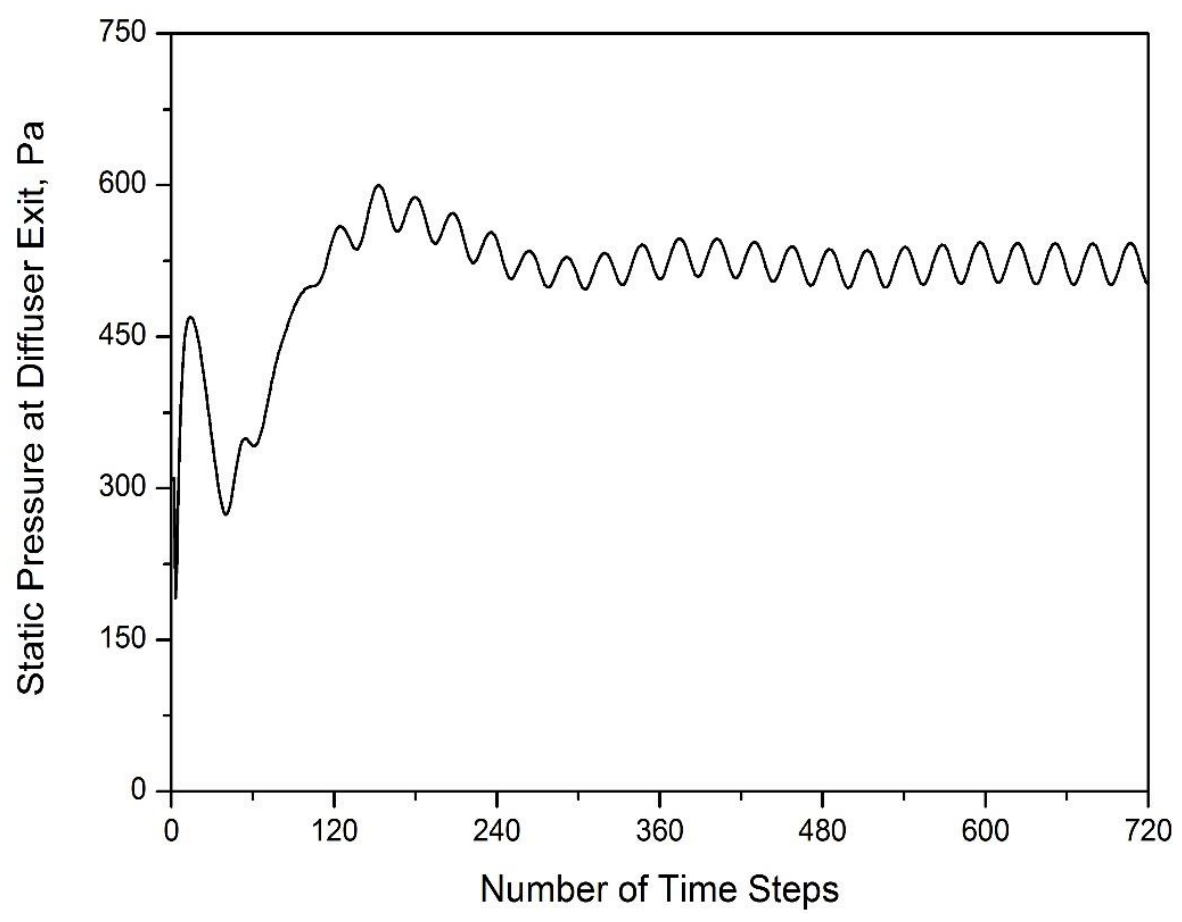

Figure 6: Pressure fluctuation graph captured at the exit of the diffuser up to two rotations of the impeller

A pre-set limiting value of 0.0001 is provided for all the residuals as a primary requirement for convergence. Every time step in the sliding mesh analysis is run with twenty computations iteratively. The weighted average values of velocity and pressure taken at salient locations in the flow domain are captured corresponding to every one degree of rotation of the impeller using time step advancement set up for the analysis. 


\subsection{Description of Performance Parameters adopted in the Analysis}

A non-dimensionalized parameter namely hub effectiveness $\left(\varepsilon_{h}\right)$ is defined as the ratio of stagnation pressure loss for the hub model to that for the hub-less base configuration. This is considered as a local variable taking care of stagnation pressure loss between inlet to the fan inlet and exit of the impeller. It is defined by Eq. (5).

$$
\varepsilon_{h}=\frac{p_{t 2}-p_{t 1 \text { with hub }}}{p_{t 2}-p_{t 1 \text { without hub }}}
$$

Two global parameters are defined, namely the static pressure rise coefficient $(\zeta)$ and the total pressure loss coefficient $(\omega)$, in order to describe the overall performance of centrifugal fan. These are defined by Eq. (6) and Eq. (7) respectively.

$$
\begin{aligned}
& \zeta=\frac{1}{N} \sum_{j=1}^{j=N}\left(\frac{p_{4}-p_{2}}{p_{t 2}-p_{2}}, t_{\text {initial }}+{ }_{j} \Delta_{t}\right) \\
& \omega=\frac{1}{N} \sum_{j=1}^{j=N}\left(\frac{p_{t 2}-p_{t 4}}{p_{t 2}-p_{2}}, t_{\text {initial }}+{ }_{j} \Delta_{t}\right)
\end{aligned}
$$

where, generally $p=\frac{1}{N} \sum_{j=1}^{j=N} p($ area node,$j)$

The performance variables namely volume and head coefficients are calculated using Eq. (8) and Eq. (9) respectively as shown below.

$$
\begin{aligned}
& \phi=\left(\frac{Q}{\pi R_{o}^{2} U_{2}}\right) \\
& \psi=\left(\frac{p_{3}}{\rho U_{2}^{2}}\right)
\end{aligned}
$$

So also the relative theoretical efficiency is defined as the ratio of energy transfer obtainable through CFD analysis to that obtainable from Euler's turbine equation and is given as, 


$$
\eta_{R t}=\left(\frac{H_{C F D}}{H_{t h}}\right)
$$

\subsection{Validation of the model}

The numerical simulation is carried out on the hub-less base model to obtain the head and volume coefficient as described in the numerical formulation. To establish validation study, the same input parameters as used in the experimental work are employed for the numerical formulation as well. The characteristic head versus volume coefficient graph is plotted for experimental and corresponding numerical results. This is shown in Fig. 7.

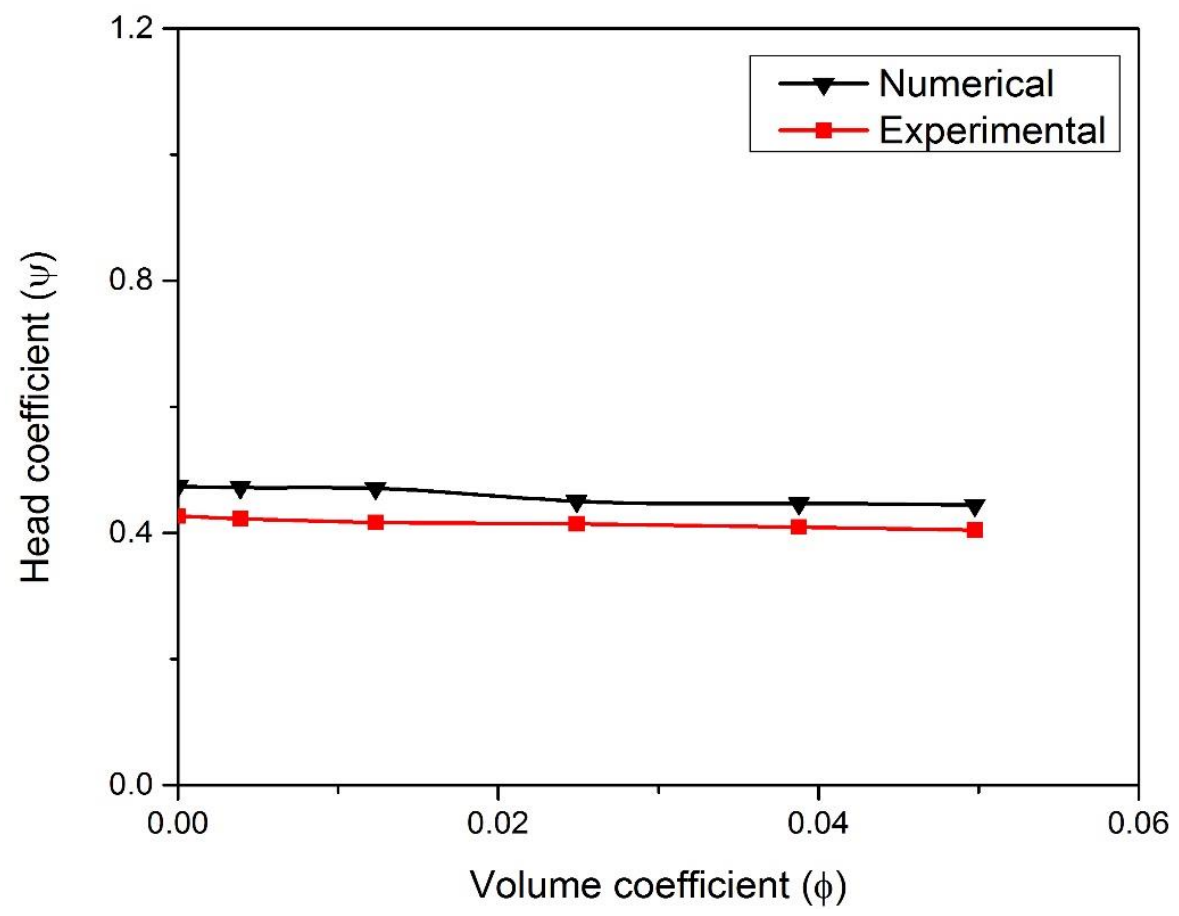

Figure 7: The main characteristics for validation study

In the experimental work corresponding to the maximum volume coefficient (fully open butterfly valve position) a head coefficient of 0.41 was obtained whereas in the numerical analysis it is found to be 0.44 . Corresponding to the shut off condition (fully closed butterfly valve position) a shut off head equivalent to 0.43 was observed for the experimental work whereas it is found to be 0.47 corresponding to numerical work. 
So also, for a robust validation study the relative theoretical efficiency is plotted between numerical and experimental results as shown in Fig 8. In the experimental work corresponding to the maximum volume coefficient (fully open butterfly valve position) a relative theoretical efficiency of $49 \%$ was obtained whereas in the numerical analysis it is found to be $54 \%$. Corresponding to the shut off condition (fully closed butterfly valve position) the relative theoretical efficiency equivalent to $63 \%$ was observed for the experimental work whereas it is found to be $70 \%$ corresponding to numerical work.

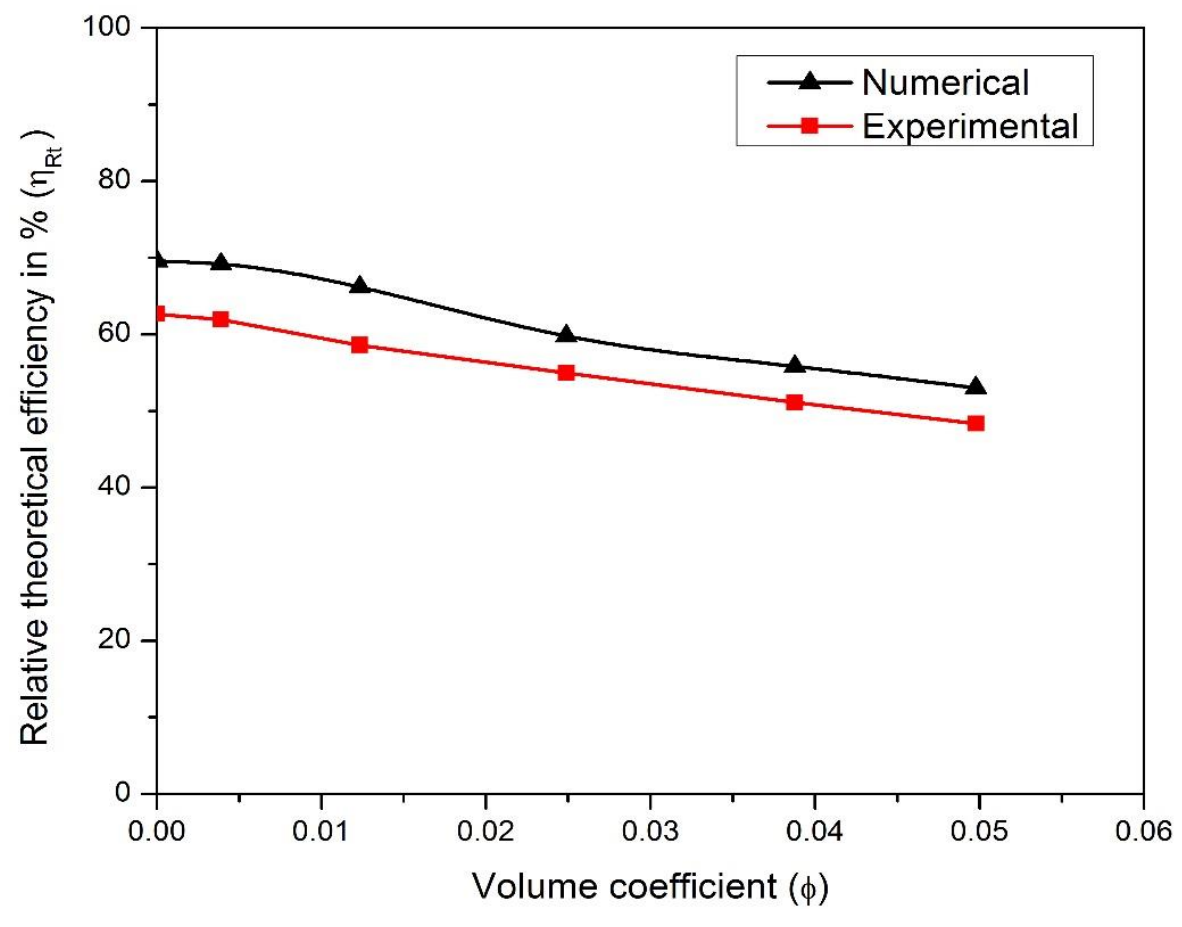

Figure 8: The operating characteristics for validation study

The error calculations between numerical and experimental results are shown in Table 3 . The $3 \sigma$ confidence limit for the error between the experimental values and numerical values ranges from $2.41 \%$ to $6.36 \%$ for head coefficient whereas the $3 \sigma$ confidence limit for the errors between the experimental and numerical values for relative theoretical efficiency varies from $2.15 \%$ to 9.83 $\%$. 


\section{Table 3: Error analysis for validation study}

\begin{tabular}{|c|c|c|c|c|c|c|}
\hline \multirow{2}{*}{$\begin{array}{c}\text { Volume } \\
\text { Coefficient } \\
(\phi)\end{array}$} & \multicolumn{3}{|c|}{ Head Coefficient $(\psi)$} & \multicolumn{3}{|c|}{$\begin{array}{c}\text { Relative Theoretical Efficiency }\left(\eta_{R t}\right) \\
\text { in } \%\end{array}$} \\
\hline & $\begin{array}{c}\text { CFD } \\
\text { Results }\end{array}$ & $\begin{array}{l}\text { Experimental } \\
\text { Results }\end{array}$ & $\begin{array}{l}\text { Absolute } \\
\text { Error }\end{array}$ & $\begin{array}{c}\text { CFD } \\
\text { Results }\end{array}$ & $\begin{array}{l}\text { Experimental } \\
\text { Results }\end{array}$ & $\begin{array}{l}\text { Absolute } \\
\text { Error }\end{array}$ \\
\hline 0.0003 & 0.4740 & 0.4270 & 0.0470 & 69.57 & 62.67 & 6.90 \\
\hline 0.0040 & 0.4720 & 0.4226 & 0.0494 & 69.19 & 61.91 & 7.28 \\
\hline 0.0130 & 0.4706 & 0.4167 & 0.0539 & 66.18 & 58.60 & 7.58 \\
\hline 0.0240 & 0.4505 & 0.4142 & 0.0363 & 59.80 & 54.99 & 4.81 \\
\hline 0.0400 & 0.4466 & 0.4090 & 0.0376 & 55.82 & 51.12 & 4.70 \\
\hline 0.0510 & 0.4440 & 0.4048 & 0.0392 & 53.02 & 48.36 & 4.66 \\
\hline \multicolumn{3}{|c|}{ RMS Value of error: } & 0.0066 & & & 1.281 \\
\hline \multicolumn{2}{|c|}{$\begin{array}{l}3 \sigma \text { limits for the } \\
\text { error }\end{array}$} & \multicolumn{2}{|c|}{$2.41 \%$ to $6.36 \%$} & \multicolumn{3}{|c|}{$2.15 \%$ to $9.83 \%$} \\
\hline
\end{tabular}

The $3 \sigma$ confidence limits imply that about $99.7 \%$ of the experimental values are in conformity with CFD analysis. The CFD results for the main characteristic graph are in fairly good agreement with the experimental results and $3 \sigma$ confidence limit for errors. So also the relative theoretical efficiency obtained from CFD study and experiments is found to be in good agreement with an average $3 \sigma$ confidence limit.

The confidence limits reasonably prove that but for slight deviations signifying losses due to leakage and other flow loss effects, the results obtained from the numerical analysis are in conformity with experimental results.

\subsection{Description of various hub geometries adopted for the numerical analysis}

In a typical hub-less base configuration flow changes its direction from axial to radial suddenly in the vicinity of impeller eye region with significant turning losses as seen in Fig. 9.

The primary aim of the present analysis is to minimize the regions of flow stalling near the inlet region of the impeller. This is accomplished by providing either a hemi-spherical or ellipsoidal shaped hub configurations as shown in figures 10 and 11 respectively. Numerical analyses for both hemi-spherical shaped hub (Fig 10) and ellipsoidal shaped hub (Fig 11) are performed to 
explore the efficacy of hub in each case vis-à-vis the hub-less base configuration. Figures 10(B) and 11(B) depict corresponding numerical models of inlet region equipped with hub. The hub is given a 'moving wall boundary condition' available in CFD software to facilitate its rotation relative to the impeller.

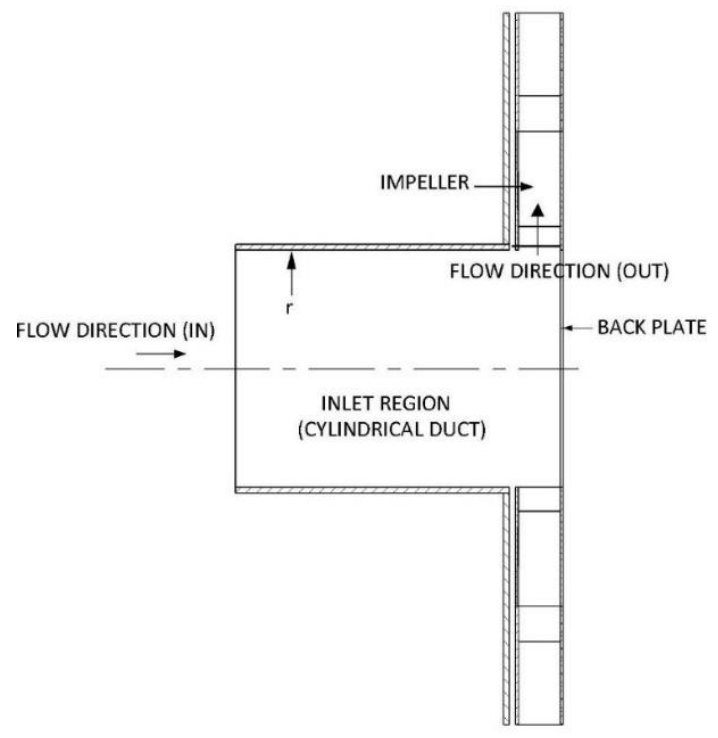

Figure 9 (A)

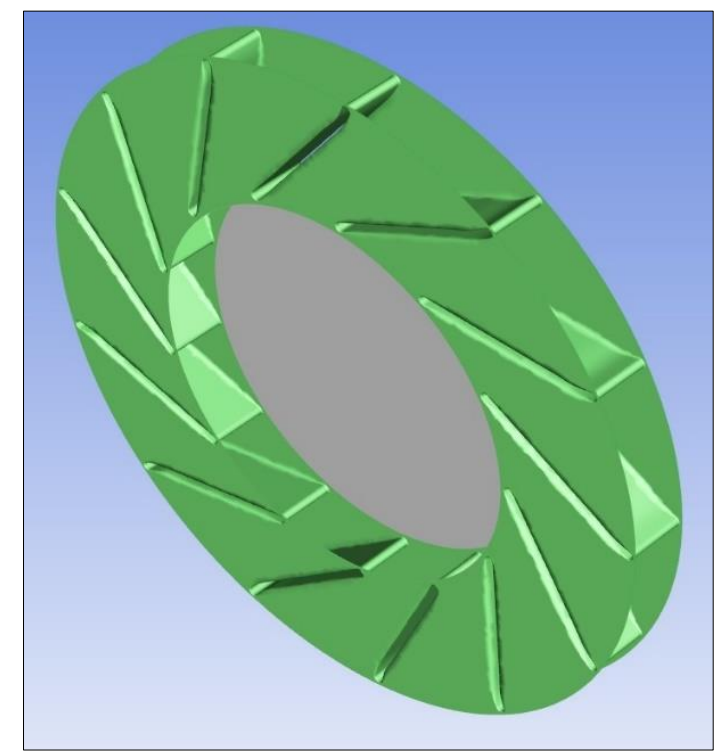

Figure 9 (B)

Figure 9: Geometric configuration for the hub-less configuration

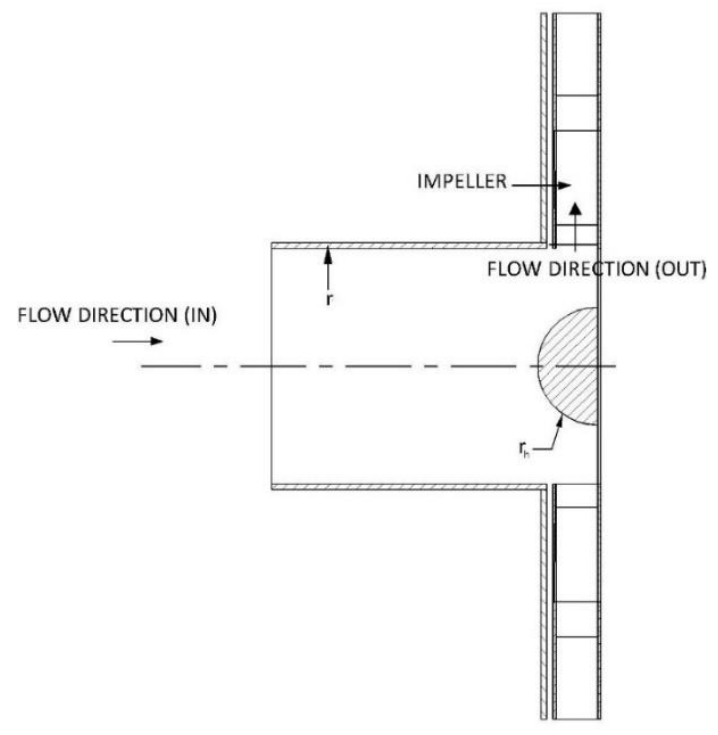

Figure $10(\mathrm{~A})$

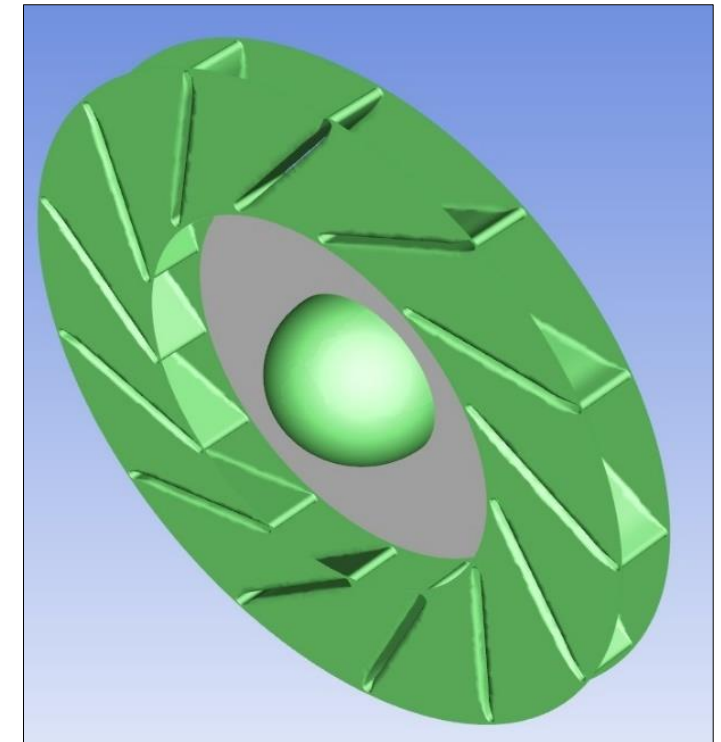

Figure $10(\mathrm{~B})$

Figure 10: Geometric configuration for the hemi-spherical hub configuration 


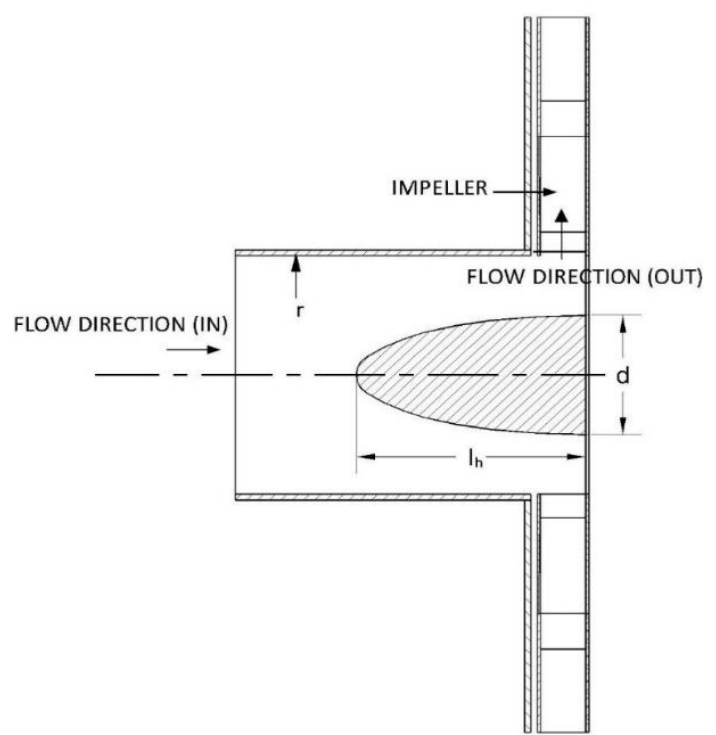

Figure $11(\mathrm{~A})$

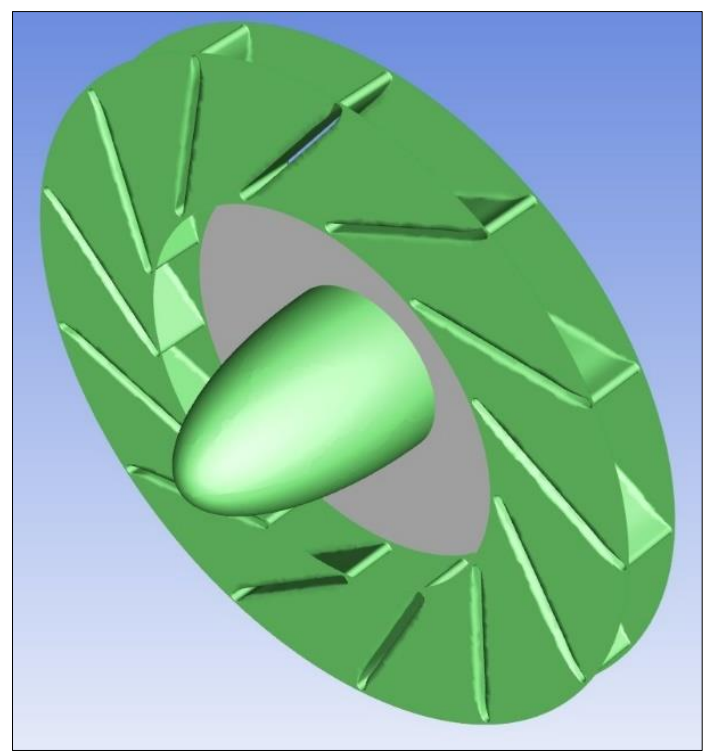

Figure $11(\mathrm{~B})$

Figure 11: Geometric configuration for the ellipsoidal hub configuration

The geometry of the hemi-spherical hub configuration is varied in terms of its hub radius and the parameter chosen for variation in ellipsoidal hub configurations is the hub length in the axial direction. These two parameters are non-dimensionalized with respect to entrance duct radius and are named spheroidal hub ratio and ellipsoidal hub ratio for hemi-spherical and ellipsoidal hub models respectively.

Spheroidal hub ratio, $R_{s}=\frac{r_{h}}{r}$

Ellipsoidal hub ratio, $R_{E}=\frac{l_{h}}{r}$

The values of spheroidal hub ratio and ellipsoidal hub ratio are parametrically varied in the analysis and are tabulated in Table 4. Meridional height of the ellipsoidal hub (d) is obtained by optimizing the radius of hemi-spherical hub geometry as explained further in section 3.1. 
Table 4: Parametric variations of spheroidal hub ratio and ellipsoidal hub ratio adopted for the analysis

\begin{tabular}{|c|c|c|c|}
\hline \multicolumn{2}{|c|}{ Hemi-spherical hub } & \multicolumn{2}{c|}{ Ellipsoidal hub } \\
\hline $\begin{array}{c}\text { Configuration } \\
\text { name }\end{array}$ & $\begin{array}{c}\text { Spheroidal } \\
\text { hub ratio }\end{array}$ & $\begin{array}{c}\text { Configuration } \\
\text { name }\end{array}$ & $\begin{array}{c}\text { Ellipsoidal } \\
\text { hub ratio }\end{array}$ \\
\hline Base Model & 0.0 & Base Model & 0.0 \\
\hline S1 & 0.3 & E1 & 0.3 \\
\hline S2 & 0.4 & E2 & 0.5 \\
\hline S3 & 0.5 & E3 & 0.7 \\
\hline S4 & 0.6 & E4 & 0.9 \\
\hline S5 & 0.7 & E5 & 1.1 \\
\hline S6 & 0.8 & E6 & 1.3 \\
\hline
\end{tabular}

\section{Results and Discussion}

To bring out the efficacy of the hub geometry on the flow characteristics in a meaningful way, an effectiveness of the hub $\left(\varepsilon_{n}\right)$ is defined as given in Eq. (5) and is used for comparing various parametrically varied hub configurations vis-à-vis the hub-less base model.

Further, to corroborate the usefulness of the hub with respect to overall improvement in terms of static pressure gain across the fan, two global output parameters namely static pressure rise coefficient $(\zeta)$ and total pressure loss coefficient $\left({ }^{\omega}\right)$ are used. These output parameters are estimated corresponding to a range of volume coefficients from 0.004 to 0.051 . The variations in the output parameters corresponding to different hub geometry configurations are presented through various histograms. Velocity contour plots are drawn to gain better understanding of the physics of flow in conjunction with the performance characteristics. The efficacy of the hub modifications on performance is studied corresponding to the design flow condition with respect to best efficiency point operation of the fan. This is followed by a discussion on the hub effectiveness corresponding to off-design flow conditions as well. 


\subsection{Fan performance due to hub geometry treatment for design condition:}

The fluid flowing in the inlet passage tends to stall on the back plate as shown in Fig. 12 (A) and (B). It shows vividly the nature of flow for the hub-less base model at the inlet eye region of the fan. It is observed that the effect of such low pressure stall distortions give rise to a more pronounced disturbed velocity field with which the fluid enters the impeller. This needs to be reduced by suitable geometric intervention of the eye region.

Hence different hub configurations are studied with the spheroidal hub ratios ranging from 0.3 to 0.8 and ellipsoidal hub ratios ranging from 0.3 to 1.3 to assess an optimum geometric hub model that would ease the inlet flow distortions and eventually make it nearly streamlined.

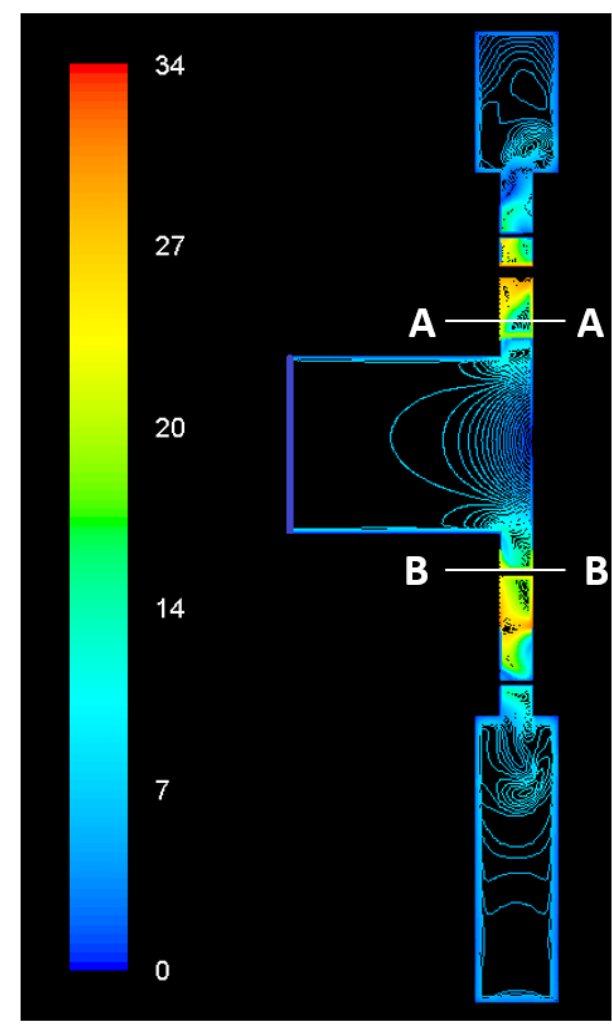

Figure 12 (A): Profile view

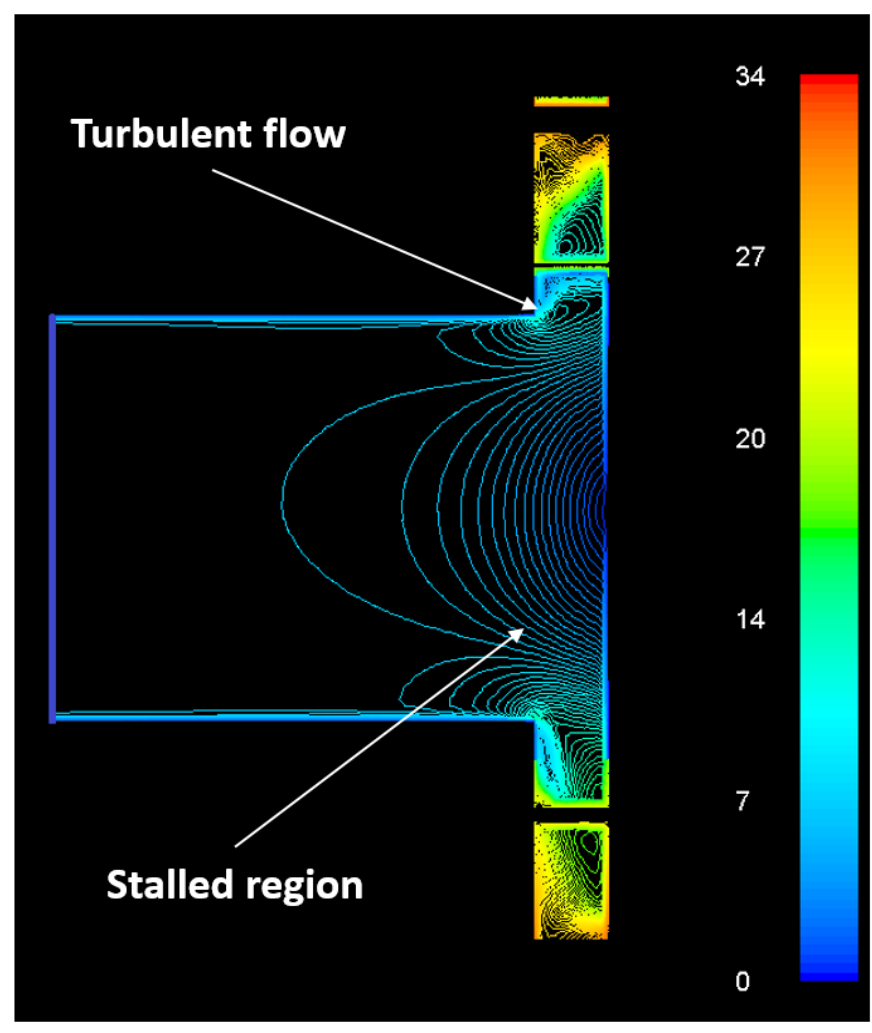

Figure 12 (B): Magnified view across Section AA $\mathrm{BB}$

Figure 12: Velocity contour plot for the hub-less base configuration

Figures 13 and 14 show the variation of useful hub effectiveness for hemi-spherical and ellipsoidal hub geometry configurations respectively at the designed volume coefficient of 0.051 . 
It is found from the analysis that a better hub effectiveness is obtained for all configurations of hemi-spherical and ellipsoidal hub geometries when compared with that of the hub-less base model. An improvement of $1 \%$ and $1.7 \%$ in hub effectiveness over the hub-less base model is observed for configuration S3 and configuration E5 respectively.

This can be attributed to the fact that the presence of a hemi-spherical or ellipsoidal shaped hub negates the stalling effect observed for the hub-less base model and provides better guidance of the flow into the meridional inlet passage of the impeller. It is to be stated here that the major flow losses are due to stalling at the hub wall as well as due to turbulent nature of the flow at the entrance to the impeller due to swirling of incoming fluid. As a consequence, it may be explained that the flow losses associated with turbulence are reduced for these configurations leading to better overall performance of the fan.

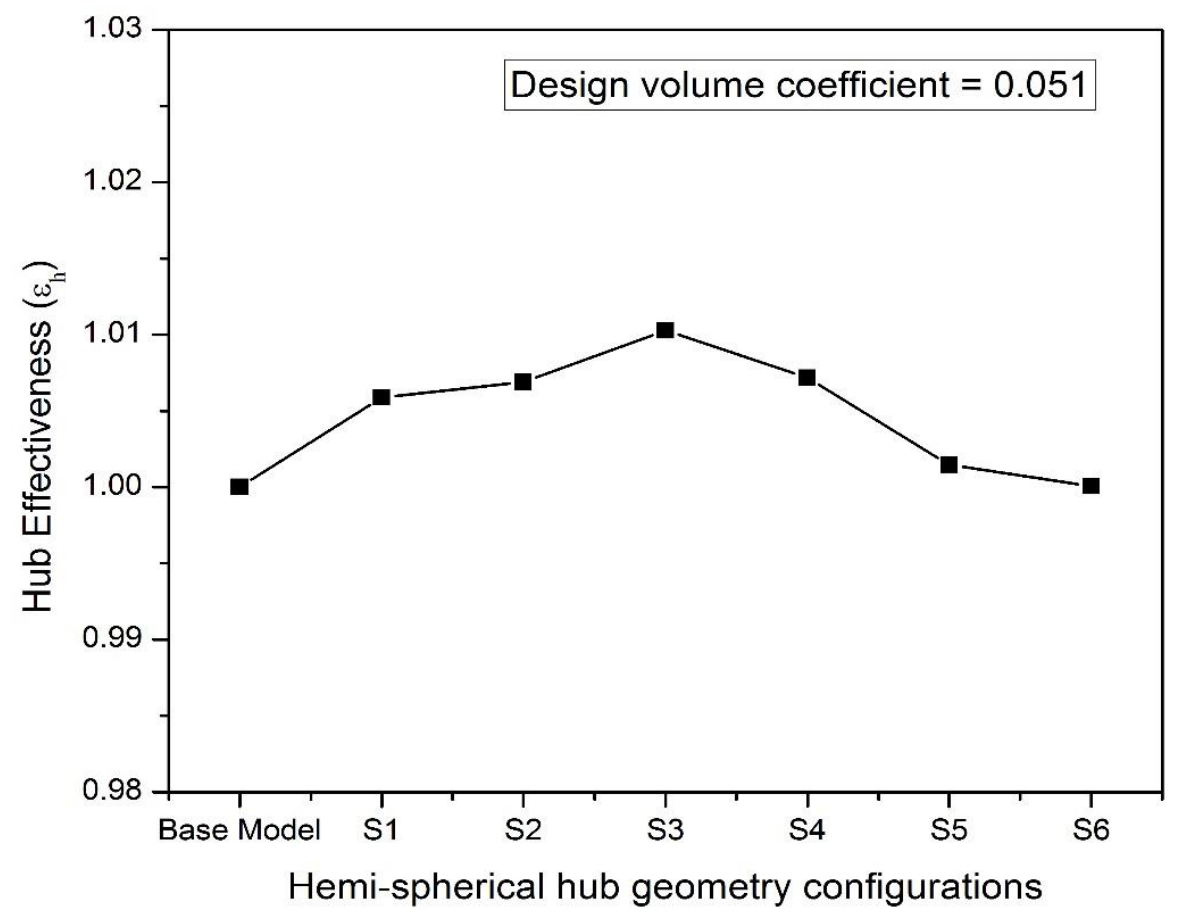

Figure 13: Hub effectiveness for various hemi-spherical hub geometry configurations 


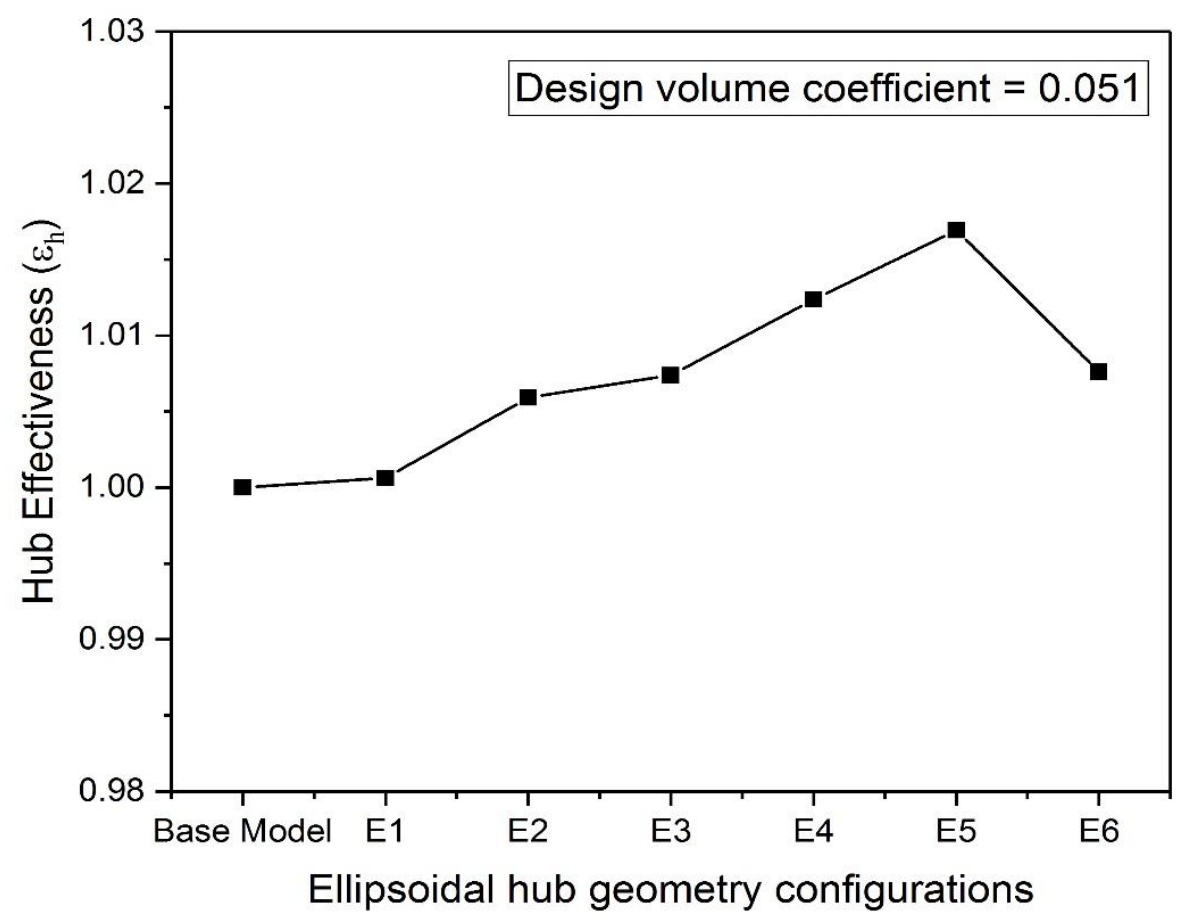

Figure 14: Hub effectiveness for various ellipsoidal hub geometry configurations

Moreover, specifically for configuration S3 corresponding to the spheroidal hub ratio of 0.5 and for configuration E5 corresponding to the ellipsoidal hub ratio of 1.1 hub effectiveness appears to be the maximum. This can be ascribed to the fact that for configurations S3 and E5, not only a streamlined through flow is observed as explained earlier but also useful acceleration is achieved which tends to guide the flow effectively by flushing the swirling eddies. As a result, it is discernable that there is a significant suppression of possible flow losses, especially stalling losses, near the inlet of the impeller when compared to the hub-less base model for these configurations as shown in Figures 15(A) and 15(B) respectively. Hence it is evident that these configurations seem to be the best hub designs among the configurations analyzed for better static pressure recovery. 


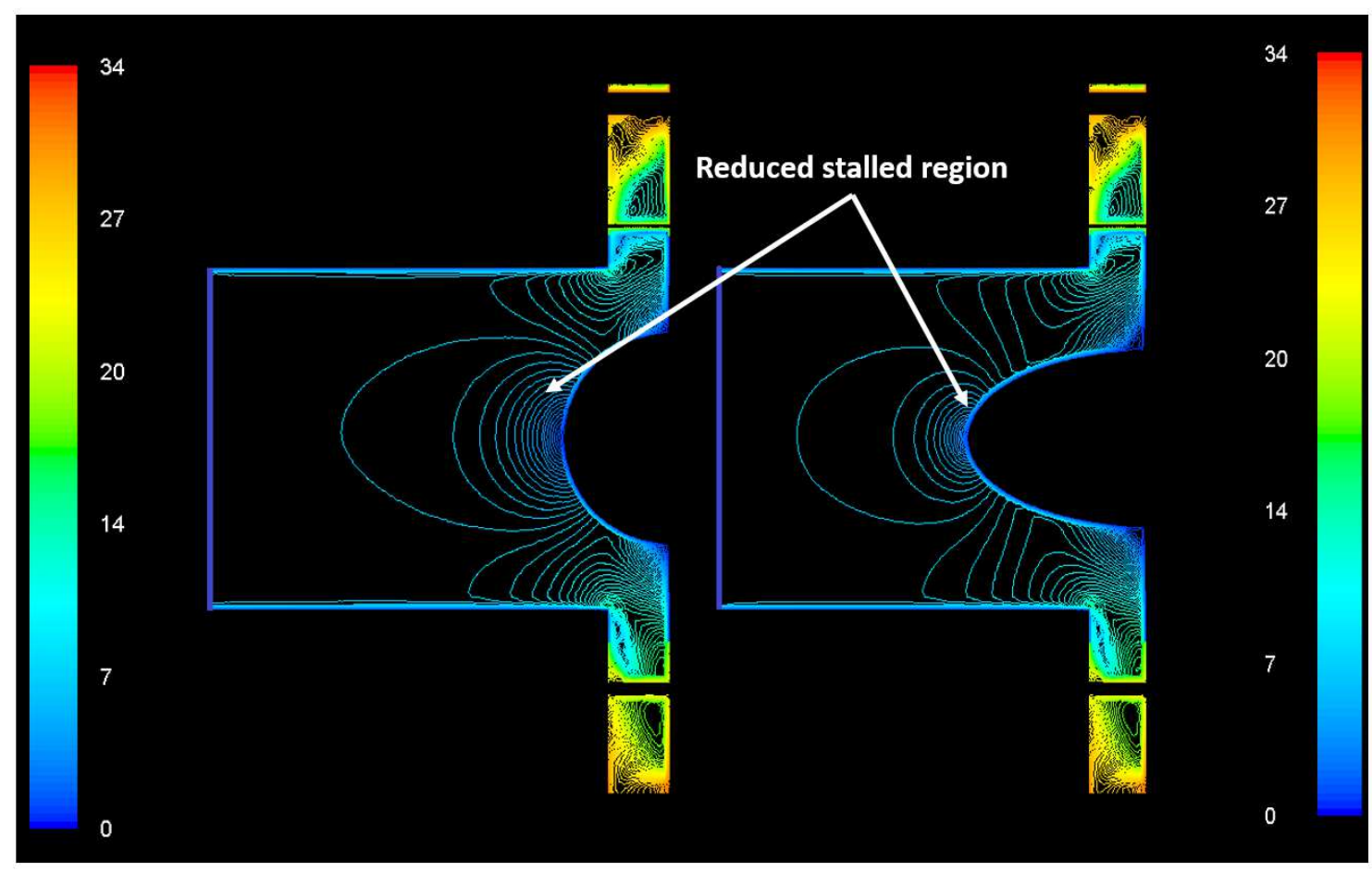

Figure $15(\mathrm{~A})$ : Configuration S3 $\left(\mathrm{R}_{\mathrm{s}}=0.5\right)$

Figure 15 (B): Configuration E5

$\left(\mathrm{R}_{\mathrm{E}}=1.1\right)$

Figure 15: Velocity contour plots for optimized hub geometry configurations

However, contrastingly it is seen from Fig. 13 that there is a significant drop in useful hub effectiveness for hemi-spherical hub configurations with spheroidal hub ratios beyond 0.5. Also for hub ratios beyond 1.1 the ellipsoidal hub configurations also show a decremental trend in useful hub effectiveness as seen in Fig. 14.

The possible reasons could be that as the hub ratio is increased there is a choking effect for the through flow as the air proceeds through a narrower passage in the vicinity of eye of the impeller as shown in Figures 16(A) and 16(B) respectively. This causes a relatively stronger turbulent jet flow into the impeller leading to a "jet effect" characterized by velocity cubed losses, hence obviously performance of the fan deteriorates. 


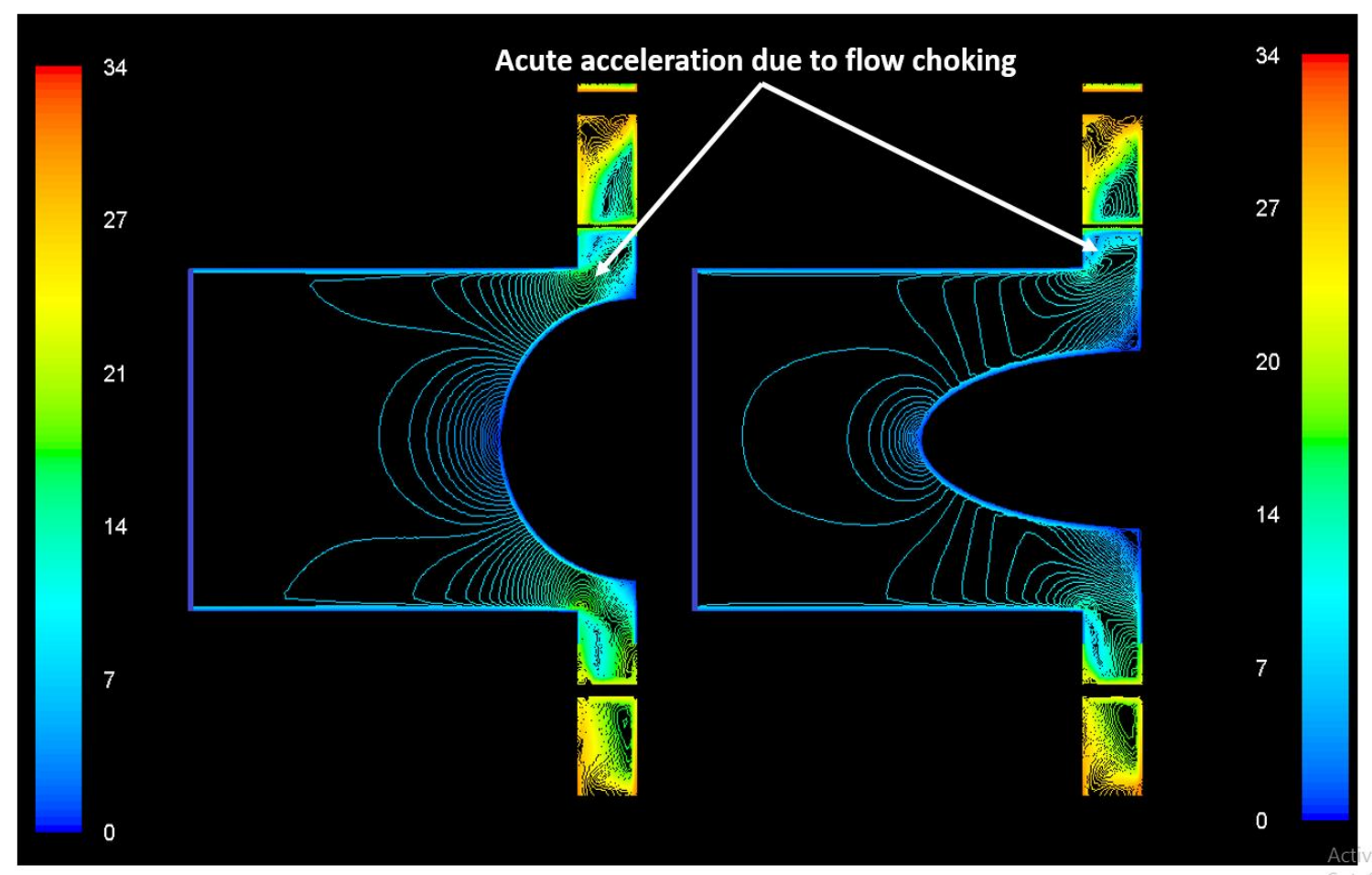

Figure 16(A): Configuration S6 $\left(\mathrm{R}_{\mathrm{s}}=0.8\right)$

Figure 16(B): Configuration E6 $\left(\mathrm{R}_{\mathrm{E}}=1.3\right)$

Figure 16: Velocity contour plots for various hub geometry configurations

Further, the results of hub treatment are corroborated using two performance variables static pressure rise coefficient and total pressure loss coefficient. Figures 17 and 18 depict the variation in static pressure rise coefficient for various configurations of hemi-spherical and ellipsoidal hub geometry respectively.

It appears that all hub geometry configurations used in the present analysis guide the flow effectively irrespective of the hub shape to reduce the inlet distortions as discussed earlier. This results in an improved flow condition contributing to lower inlet flow losses, which leads to a relatively higher static pressure rise coefficient for these configurations as shown in Fig 17 and 18 vis-à-vis the hub-less base configuration. Also, the effect of weakening of inlet distortions is manifested as a reduction in total pressure loss coefficient as shown in Fig. 19 and 20 for hemispherical and ellipsoidal hub geometry configurations respectively. 


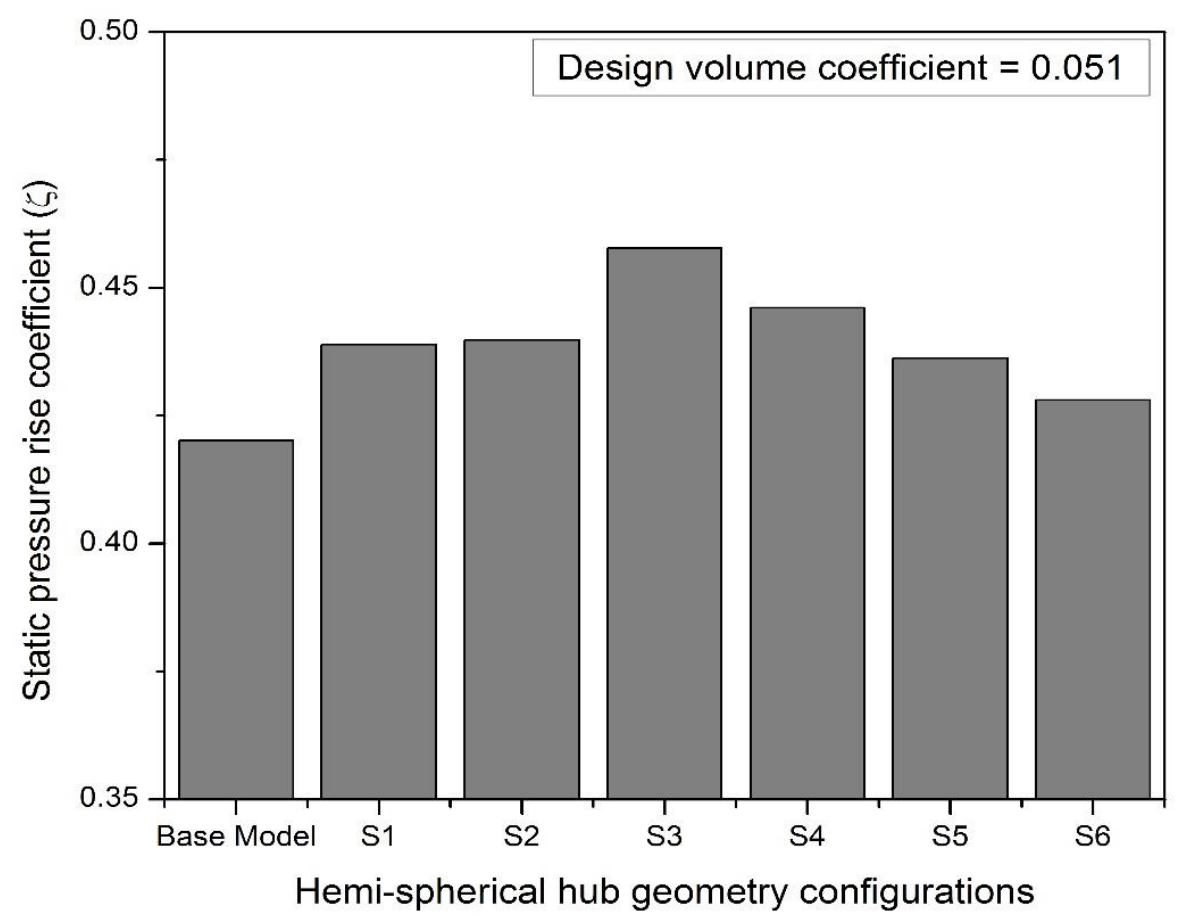

Figure 17: Static pressure rise coefficient for various hemi-spherical hub geometry configurations

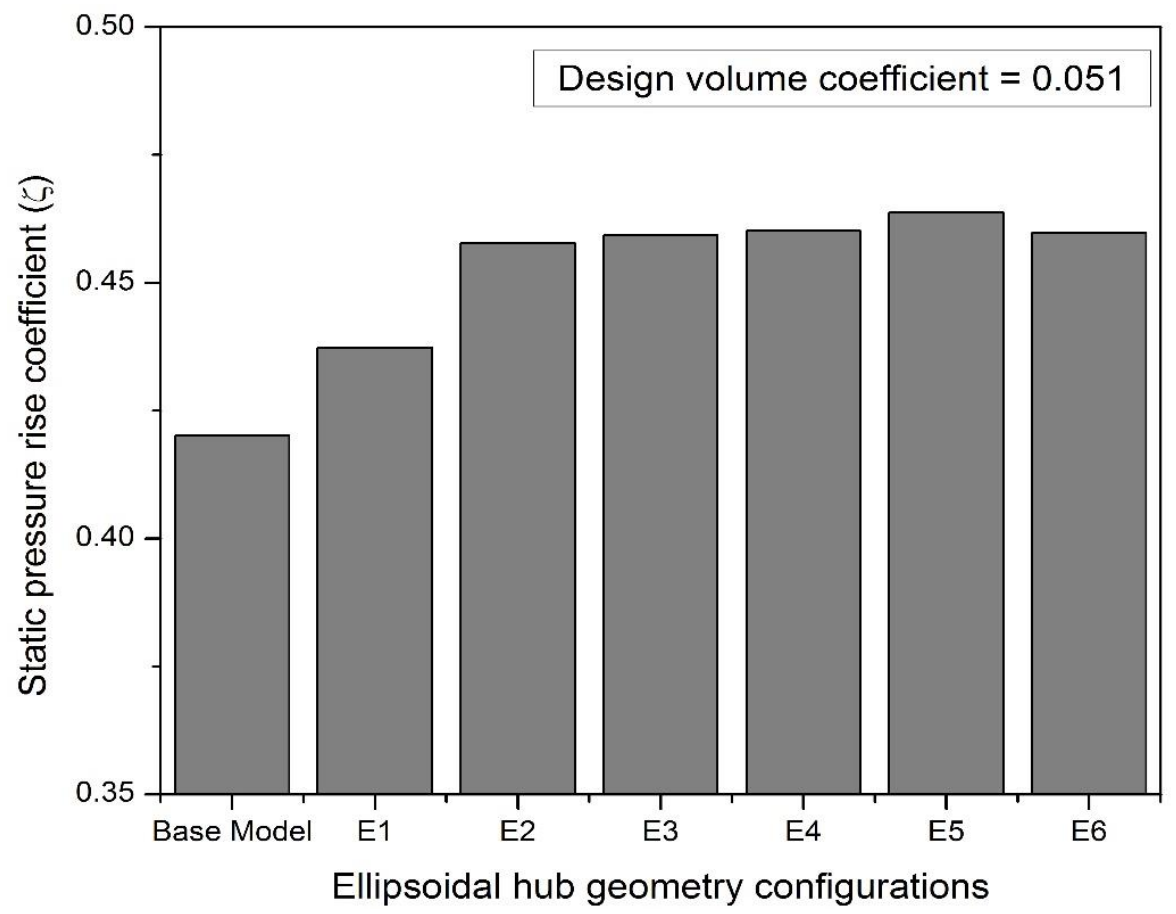

Figure 18: Static pressure rise coefficient for various ellipsoidal hub geometry configurations 


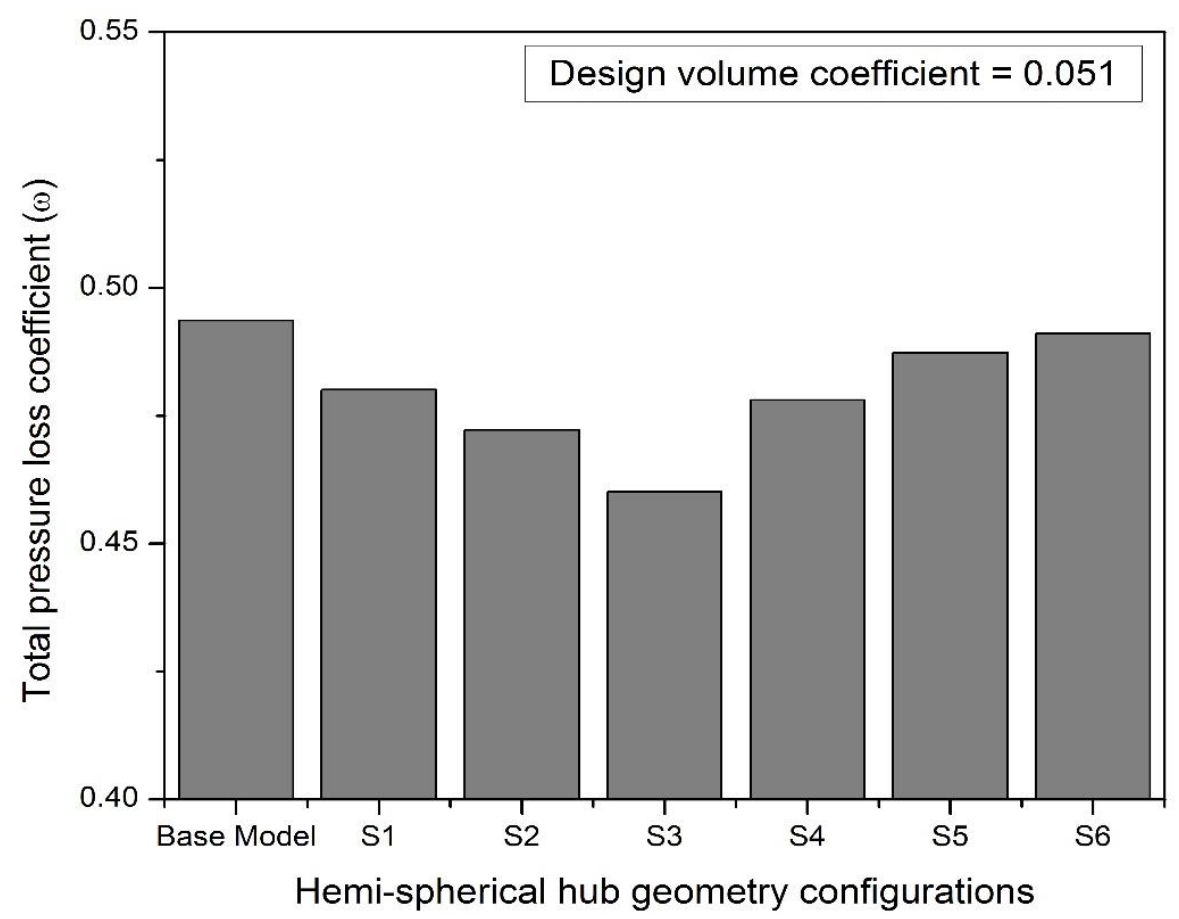

Figure 19: Total pressure loss coefficient for various hemi-spherical hub geometry configurations

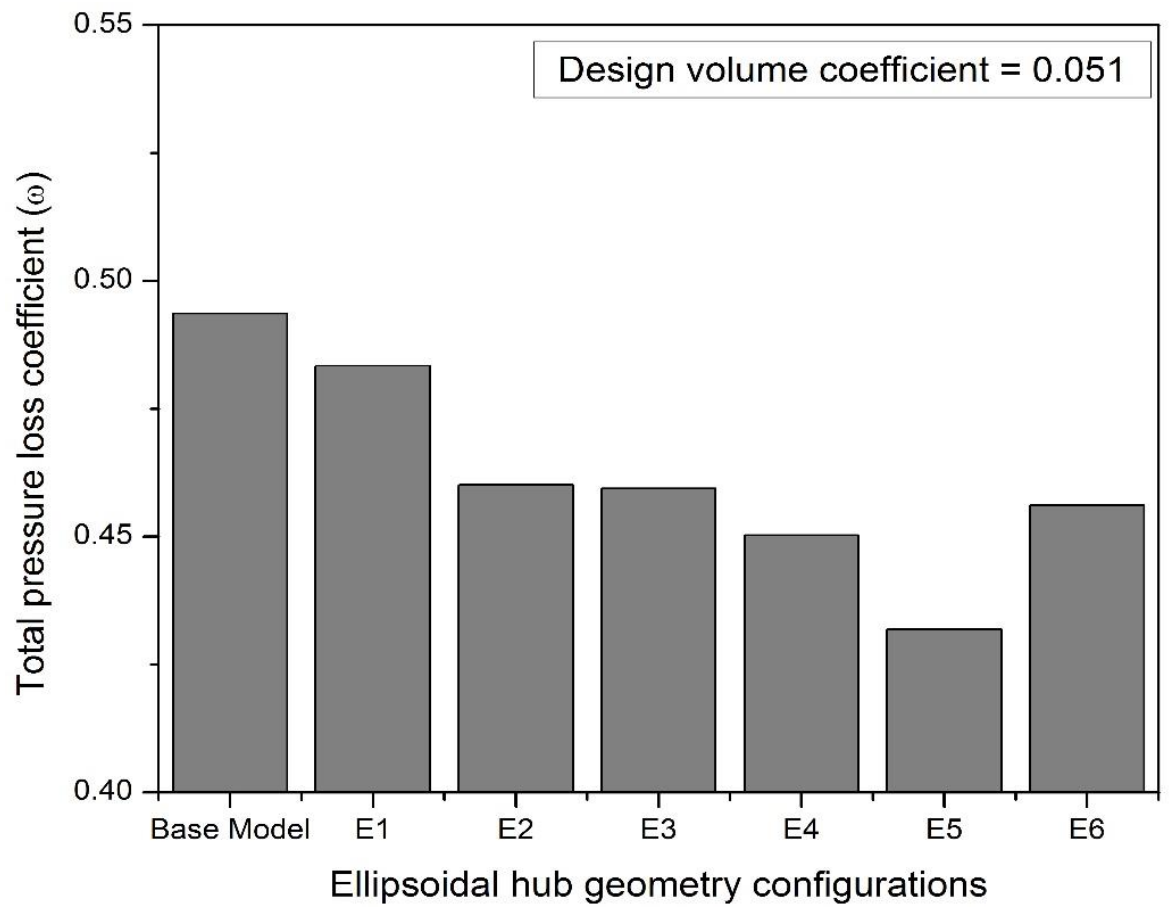

Figure 20: Total pressure loss coefficient for various ellipsoidal hub geometry configurations 
Additionally, Figures 21 and 22 show the variation in turbulent viscosity ratio corresponding to various hemi-spherical and ellipsoidal hub geometry configurations tried out in the analysis. It is seen from the Fig 21 that the turbulent viscosity ratio decreases for hemi-spherical hub geometry configurations up to the spheroidal hub ratio of 0.5 . The turbulent viscosity ratio decreases for ellipsoidal hub geometry configurations up to an ellipsoidal hub ratio of 1.1 as seen in Fig. 22. Because of this reason, the performance in terms of static pressure rise, increases for all hub geometry configurations.

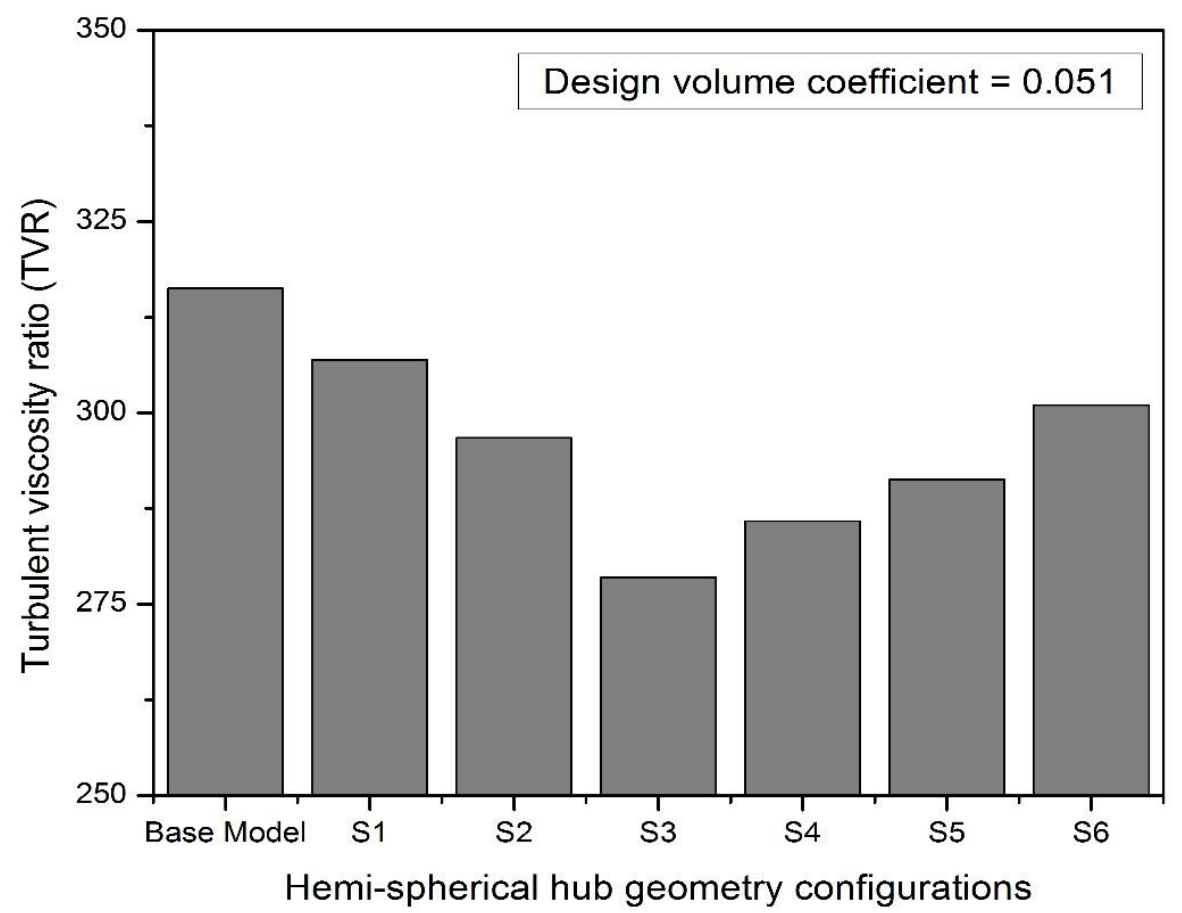

Figure 21: Turbulent viscosity ratio for various hemi-spherical hub geometry configurations 


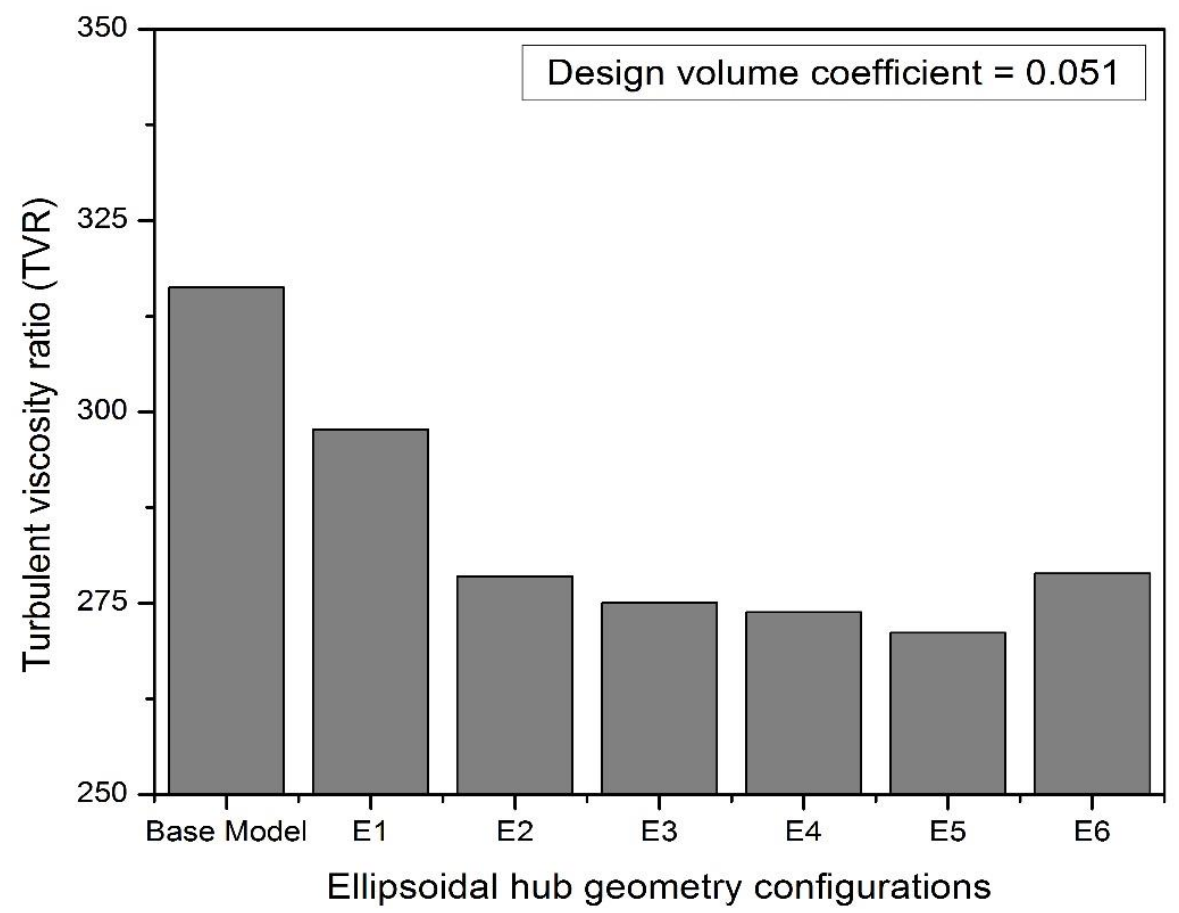

Figure 22: Turbulent viscosity ratio for various ellipsoidal hub geometry configurations

\subsection{Fan performance due to hub geometry treatment for off-design conditions:}

It is proposed to assess the effect of volume coefficient on the performance parameters corresponding to various hub geometry configurations used in the analysis. The volume coefficients considered in the analysis vary from a lower value of 0.004 to a higher value of 0.051. From Figures 23 and 24, it is clearly seen that there is a consistent decrease in the static pressure rise coefficient with increase in the volume coefficient. This is true for hub-less base configuration as well as hemi-spherical and ellipsoidal hub geometry configurations used in the study. 


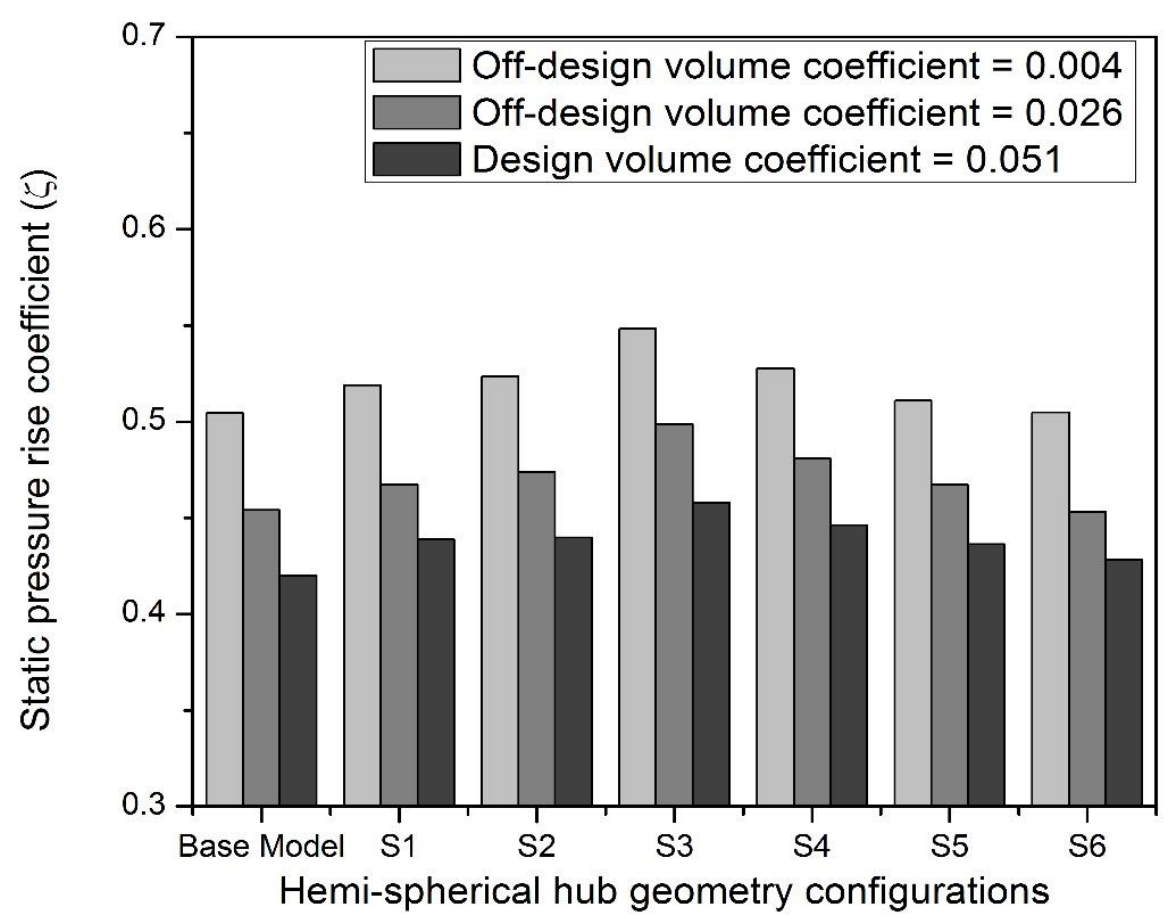

Figure 23: Static pressure rise coefficient of hemi-spherical hub geometry configurations for various volume coefficients

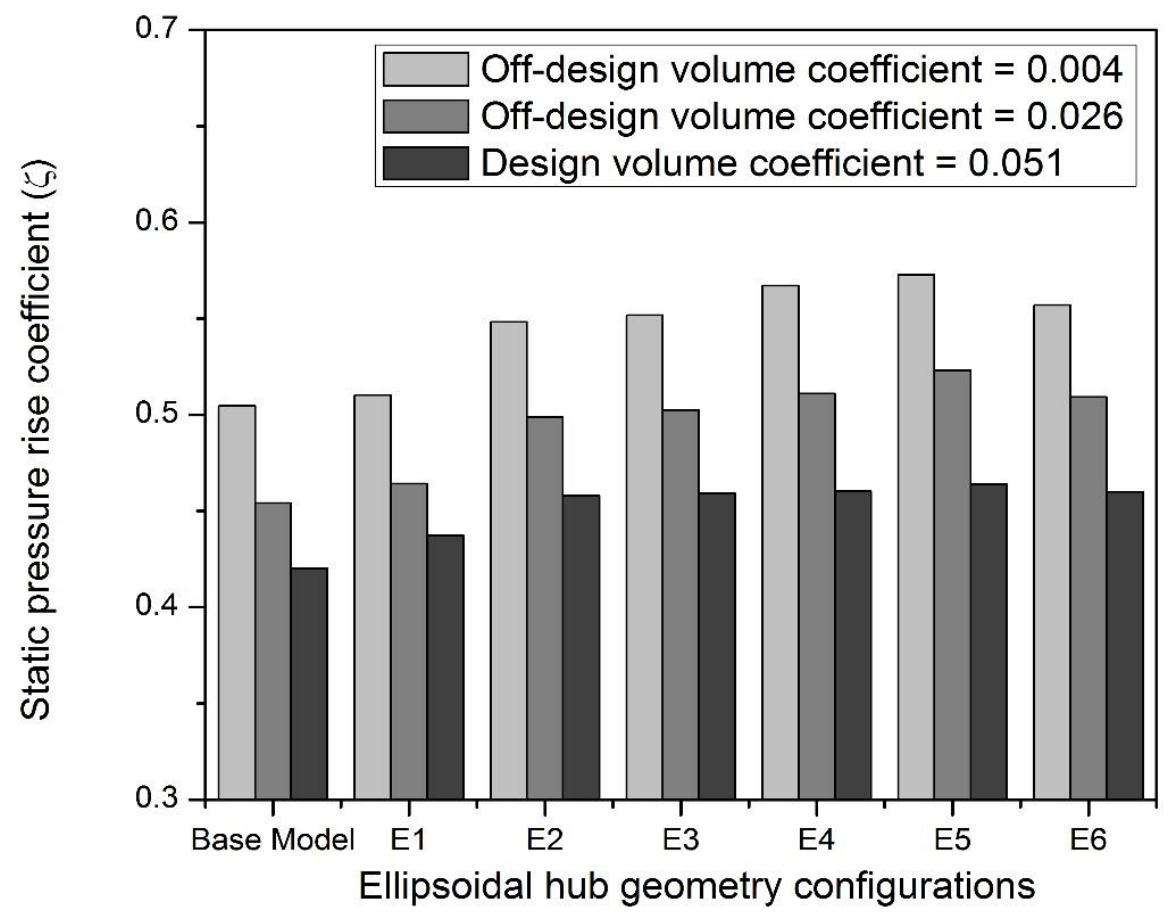

Figure 24: Static pressure rise coefficient of ellipsoidal hub geometry configurations for various volume coefficients 
It is also established that hemi-spherical hub configuration S3 and ellipsoidal hub configuration E5 appear to be the optimum design configurations providing relatively higher static pressure rise coefficient for any of the volume coefficients used in the analysis when compared to the other configurations including the hub-less base model. An improvement of about $3.27 \%$ in static pressure rise coefficient is observed for the optimized hemi-spherical hub geometry configuration S3 whereas the optimum ellipsoidal hub geometry E5 provides an improvement of about $6.07 \%$ as compared to hub-less base model. Hence it may be concluded that it is advantageous to adopt hemi-spherical hub geometry corresponding to a spheroidal hub ratio of 0.5 and ellipsoidal hub geometry corresponding to an ellipsoidal hub ratio of 1.1 for improved performance of the fan.

\subsection{Comparison of optimum hemi-spherical and ellipsoidal hub configurations}

As an objective the present study on optimum hub geometric configurations evolved for both hemi-spherical and ellipsoidal type hubs. This section describes a comparative study of fan performance for these optimum hub geometry configurations. The performance parameters chosen for this study are head coefficient and the relative theoretical efficiency.

Fig. 25 depicts the plot for head coefficient for optimum hemi-spherical hub configuration (S3) as well as ellipsoidal hub configuration (E5), vis-a-vis hub-less base configuration. It is seen that the optimum ellipsoidal configuration (E5) marginally yields better head coefficient at all volume coefficients considered. On an average, the optimum hub geometry configuration yields $8.4 \%$ higher head coefficient as compared to the hub-less base model and a significant augmentation of about $1.04 \%$ in head coefficient is obtained for configuration E5 over that of the hemi-spherical hub model (S3).

Similarly, the plot of relative theoretical efficiency for optimum hemi-spherical (S3) as well as ellipsoidal hub (E5) configurations is also shown in Fig. 25. It is observed that the trend of variation for relative theoretical efficiency follows that for head coefficient for the optimized hub configurations. On an average, it is seen that the optimum hub geometry configuration (E5) yields $8.6 \%$ higher relative theoretical efficiency as compared to the hub-less base model and an 
improvement of about $\quad 3.8 \%$ is discernable over that of the optimum hemi-spherical hub model (S3) for the range of volume coefficients considered.

It is clearly seen that the ellipsoidal hub configuration E5 appears to be better for both performance parameters. This may be inferred from the fact that the turning losses in the vicinity of eye of the impeller probably get reduced in the presence of optimized ellipsoidal hub configuration when compared to that of hemi-spherical hub model. The effects of this is contributing to an improvement in streamlining of flow at the inlet region and the overall gain across the entire fan is that of an improvement in both head coefficient and relative theoretical efficiency for E5 configuration.

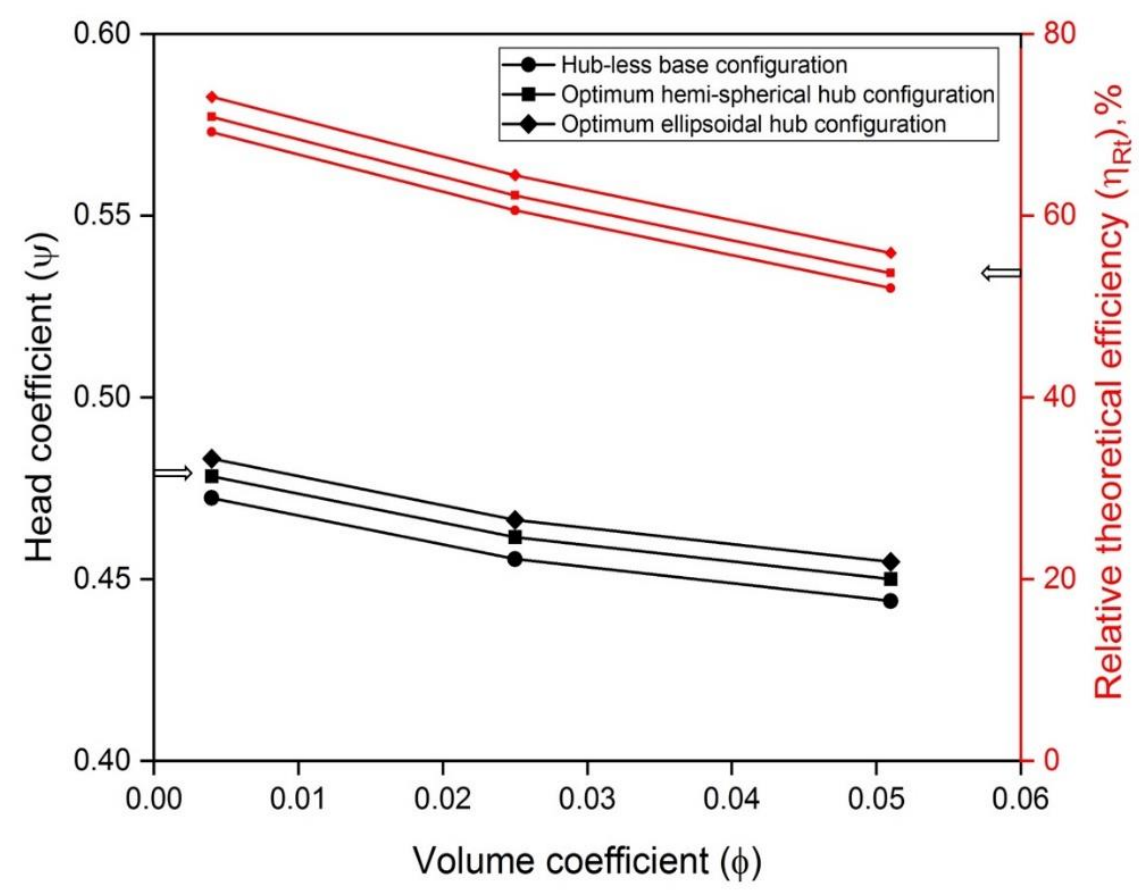

Figure 25: Performance comparison plots for optimized hemi-spherical and ellipsoidal hub configurations

\subsection{Comparison of numerical and experimental results for optimum hub configuration}

Experimental studies were conducted for all the numerical models discussed in this paper and the results of comparison between experimental and numerical studies with regards to optimum configurations are presented below. Figures 26 and 27 show the main and operating 
characteristics of the optimum hub geometry configuration (ellipsoidal hub geometry configuration E5 having $\mathrm{R}_{\mathrm{E}}=1.1$ ) for both experimental and numerical studies respectively. As observed in these figures, the experimental results are in close accordance with the results that are obtained from numerical studies. The variation between the experiment and the numerical results is as a consequence of possible uncertainty errors associated with the experimental procedures.

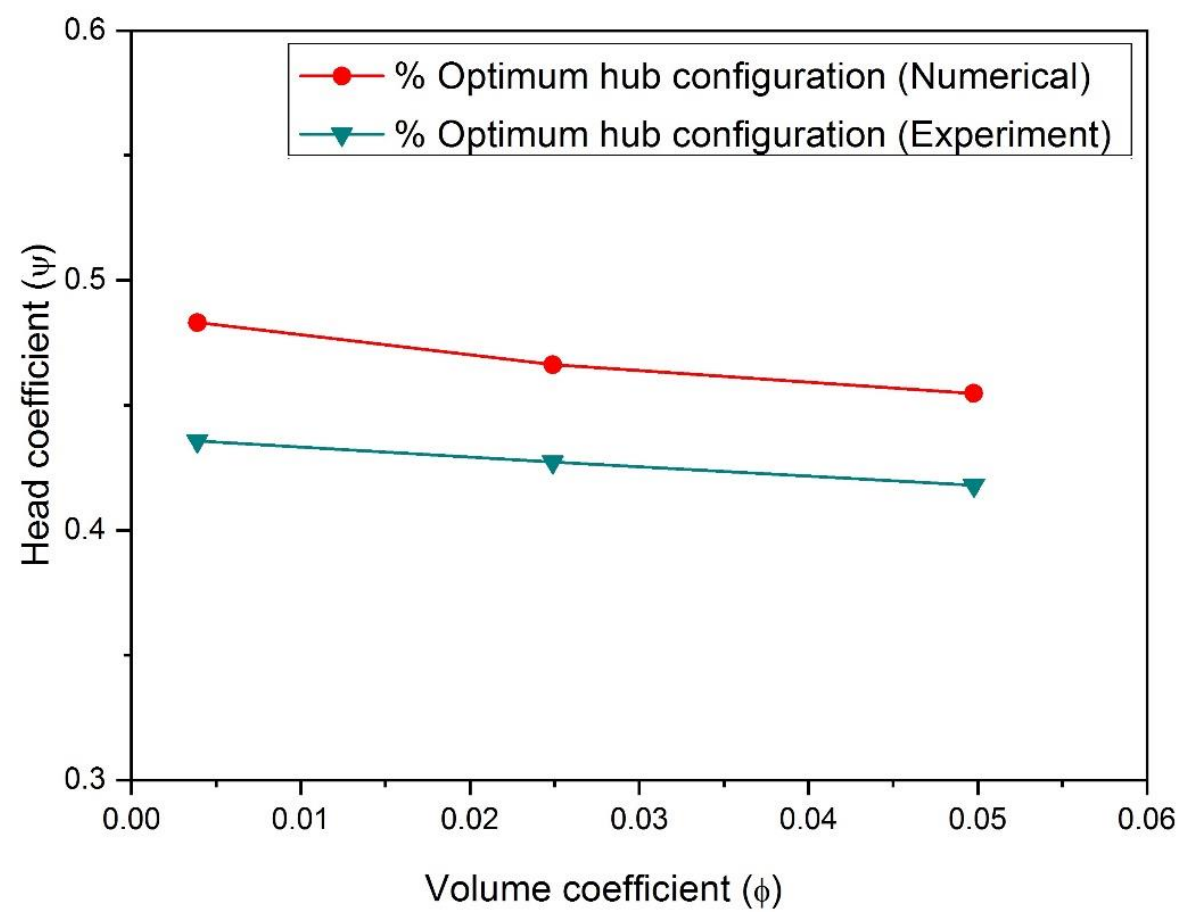

Figure 26: Comparison of head coefficient for the optimum hub geometry configuration

Thus from the experimental and numerical studies, it can be concluded that the hub configuration having ellipsoidal hub ratio of 1.1 appears to be the better configuration when compared to all other geometric configurations. Hence this geometric model can be adopted as a design prescription for a centrifugal fan hub with reasonable assurance of improved performance. 


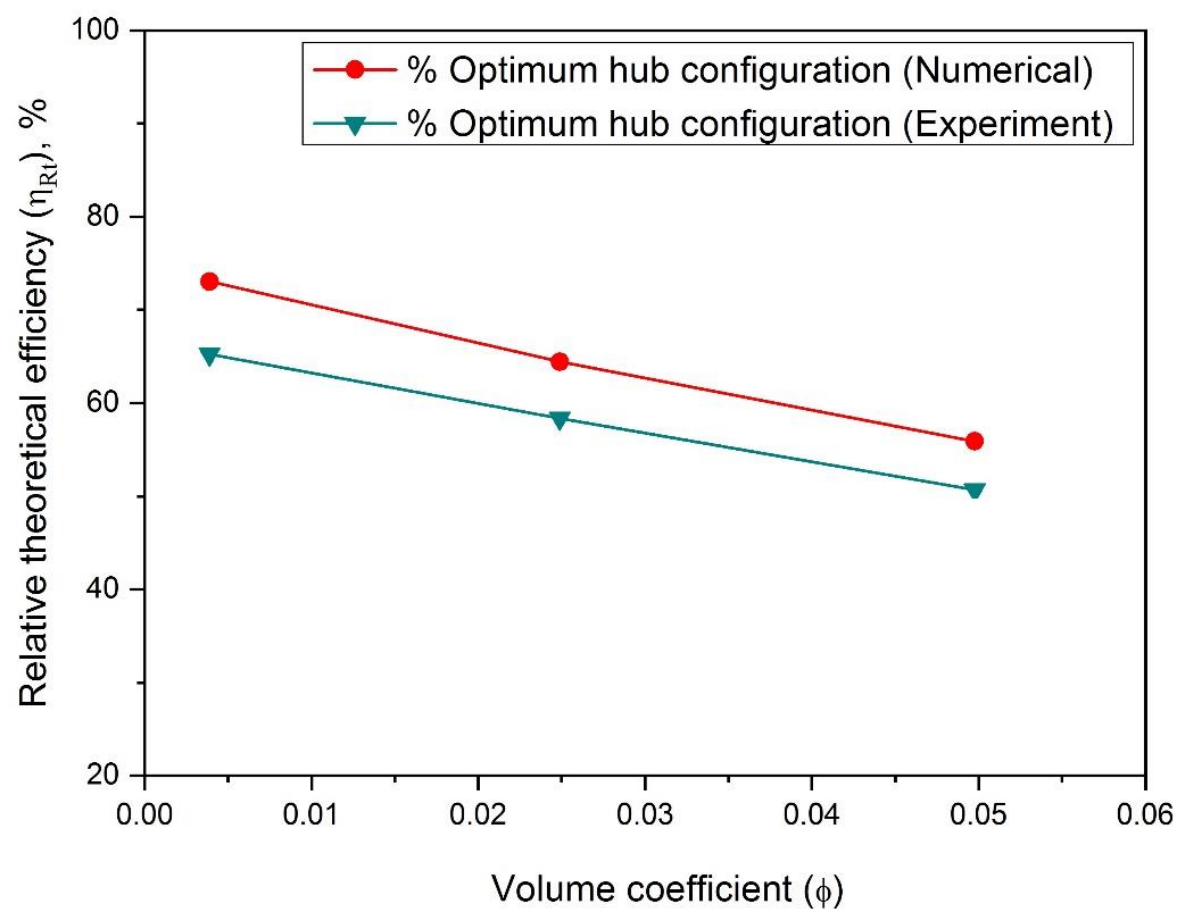

Figure 27: Comparison of relative theoretical efficiency for the optimum hub geometry configuration

\subsection{Development of correlations for the optimized hub shape configuration}

The results discussed in Section 3.3 show that the fan performance characteristics, i.e. head coefficient $(\psi)$ and relative theoretical efficiency $\left(\eta_{R t}\right)$, are significantly influenced by both volume coefficient $(\phi)$ and Ellipsoidal hub ratio $\left(\mathrm{R}_{\mathrm{E}}\right)$ considered in the study. Therefore, $\psi$ and $\eta_{R t}$ can be expressed as functions of $\phi$ and $R_{E}$ as given in the equations (13) and (14) respectively.

$\psi=f \phi, R_{E}=C_{1} \phi^{a} R_{E}^{b}$

$\eta_{R t}=f_{f} \phi, R_{E}=C_{2} \phi^{m} R_{E}^{n}$

where $\mathrm{C}_{1}, \mathrm{C}_{2}, \mathrm{a}, \mathrm{b}, \mathrm{m}$ and $\mathrm{n}$ are empirical constants.

Regression analysis of the numerically obtained values has been performed to determine the constants in the equations (13) and (14). This analysis provides the exact relationships for $\psi$ and 
$\eta_{R t}$ using least square fit and the correlations thus obtained are as shown in equations (15) and (16) respectively.

$$
\begin{aligned}
& \psi=0.43 \phi^{-0.02} R_{E}^{0.018} \\
& \eta_{R t}=42.01 \phi^{-0.01} R_{E}^{0.029}
\end{aligned}
$$

Figures 28 and 29 show comparison of numerical and predicted values of head coefficient and relative theoretical efficiency for ellipsoidal hub geometry configurations. It can be seen from these parity plots that $95 \%$ of the predicted values of head coefficient and relative theoretical efficiency (calculated using equations 15 and 16) lies within $\pm 5 \%$ of the numerically obtained values [10].

The regression data of head coefficient for the correlation obtained in Eq. (15) has regression coefficient of 0.91 and average absolute deviation of $0.9 \%$ whereas the regression data of relative theoretical efficiency for the correlation obtained in Eq. (16) has regression coefficient of 0.89 and average absolute deviation of $6.3 \%$. This depicts good agreement between predicted data and numerical data. 


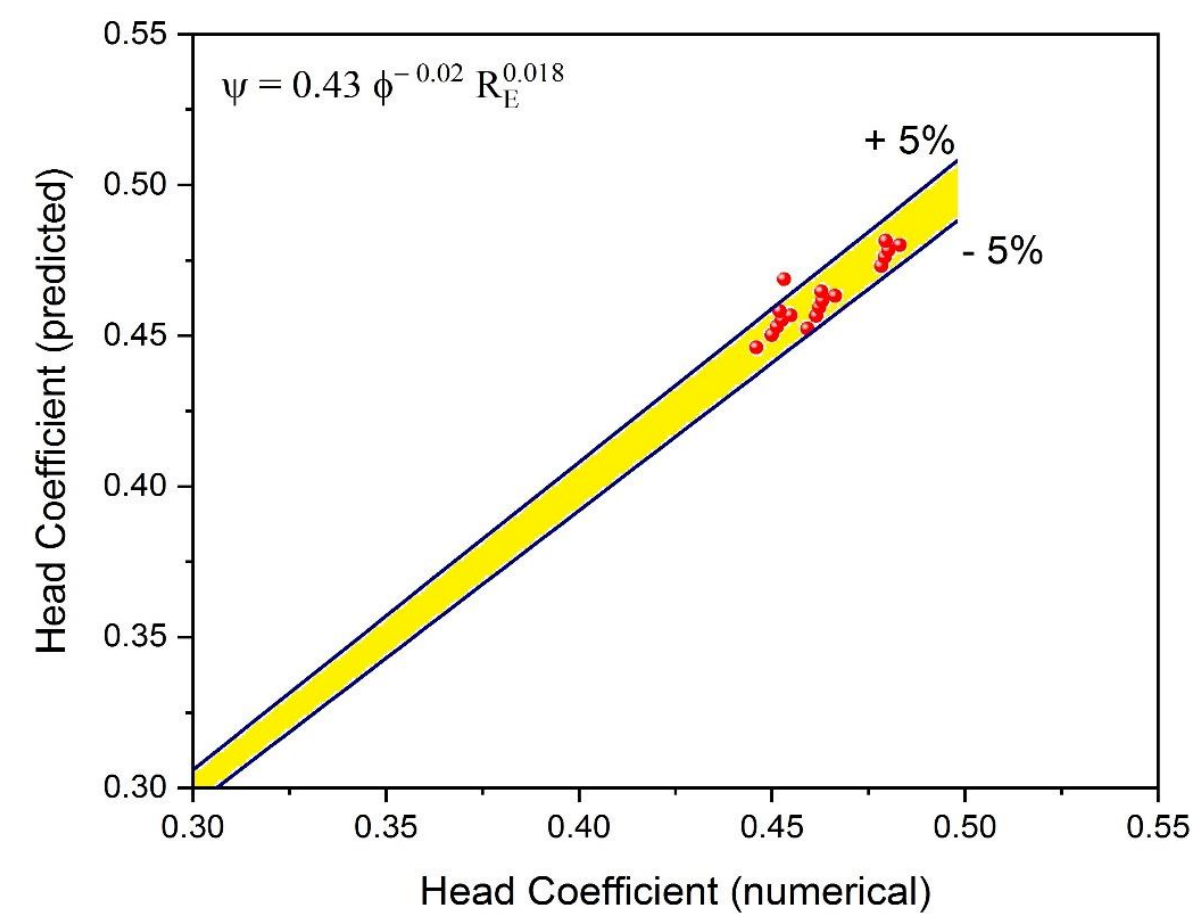

Figure 28: Comparison of numerical and predicted values of Head Coefficient for ellipsoidal hub geometry configuration

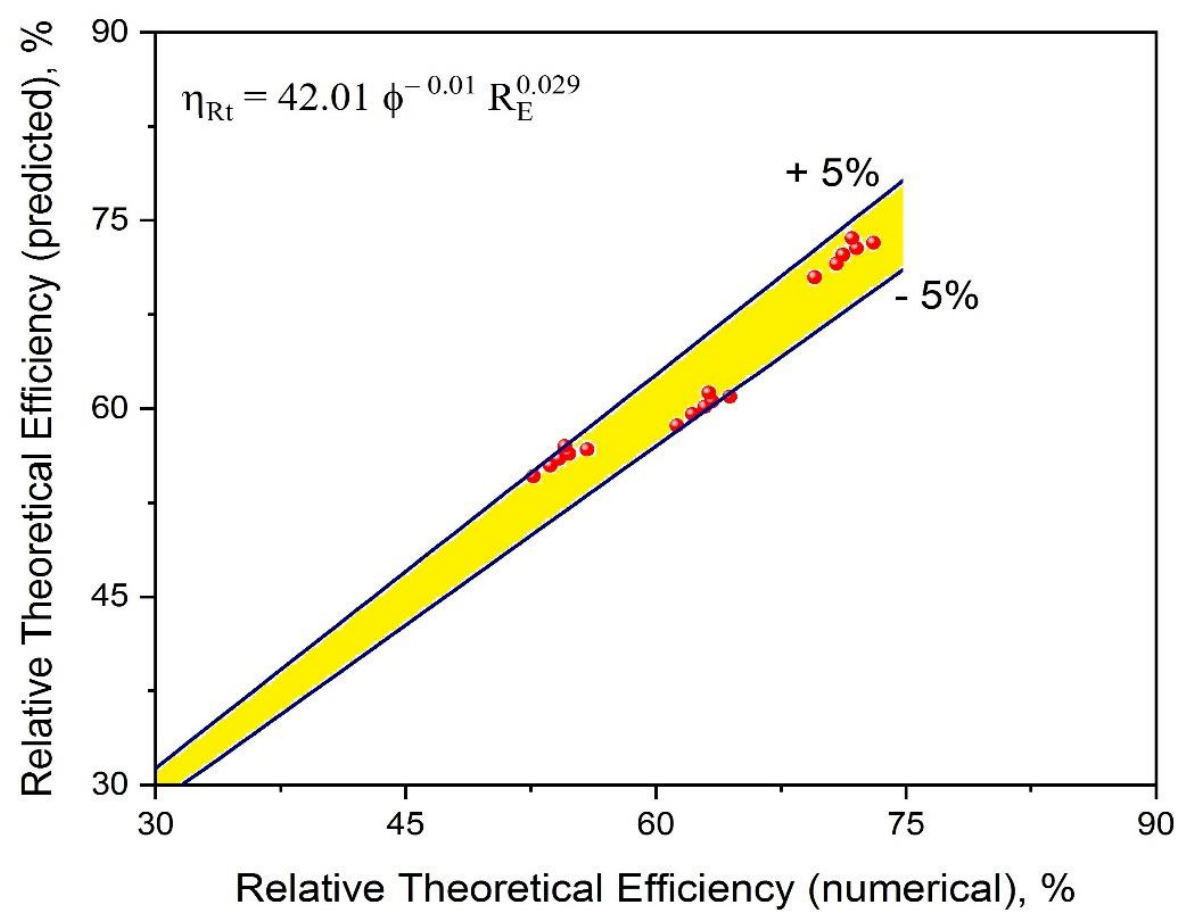

Figure 29: Comparison of numerical and predicted values of Relative Theoretical Efficiency for ellipsoidal hub geometry configuration 
In conclusion, for the range of parameters investigated in the numerical work, the values of head coefficient as well as relative theoretical efficiency can be predicted with reasonable accuracy using the correlations developed in equations 15 and 16 respectively.

\section{Conclusion}

It is revealed from numerical analysis that hub plays a vital role in streamlining the flow at the inlet to the centrifugal fan and augments its performance. Following conclusions are summarized from the present numerical analysis and comparative study.

$\square$ It is found that both hemi-spherical and ellipsoidal hub geometry configurations contribute to higher overall efficiency as compared to hub-less base model at all volume coefficients considered in the study.

Ellipsoidal hub configuration with ellipsoidal hub ratio of 1.1 contributed an improvement of about $8.4 \%$ in head coefficient and $8.6 \%$ in relative theoretical efficiency as compared to the hub-less base model for the range of volume coefficients adopted in the present study.

Hemi-spherical hub configuration with spheroidal hub ratio of 0.5 contributed to a higher head coefficient of about $2.3 \%$ and a higher relative theoretical efficiency of about $3.2 \%$ when compared to a hub-less base model at all volume coefficients.

For hub configurations with ellipsoidal hub ratio beyond 1.1 as well as spheroidal hub ratio beyond 0.5 , there was only a marginal improvement in terms of performance characteristics of the fan.

It is found from comparison of experimental and numerical study of the optimum configuration that there is a matching trend for the performance characteristics between the two analyses. 
Statistical correlations for head coefficient and relative theoretical efficiency have been developed as functions of volume coefficient and ellipsoidal hub ratio, and the correlations predict the values of head coefficient and relative theoretical efficiency with average absolute deviation of $0.9 \%$ and $6.3 \%$, respectively.

\section{Nomenclature}

D: Diameter of cylindrical inlet section $(\mathrm{mm})$

$\mathrm{d}$ : Height of ellipsoidal hub in radial direction(mm)

$\mathrm{R}_{\mathrm{s}}$ : Spheroidal hub ratio

$\psi$ : Head coefficient

$\mathrm{U}$ : Tangential speed of impeller $(\mathrm{m} / \mathrm{s})$

$\mathrm{p}_{\mathrm{t}}$ : Total pressure $(\mathrm{Pa})$

$\mathrm{R}_{0}$ : Radius of impeller at its exit (mm)

$H_{t h}:$ Euler's energy transfer $(\mathrm{kJ} / \mathrm{kg})$

$\eta_{R t}:$ Relative theoretical efficiency

$\omega$ : Total pressure loss coefficient

$\mathrm{H}_{\mathrm{CFD}}$ : Energy transfer obtained from numerical analysis $(\mathrm{kJ} / \mathrm{kg})=p_{3} / \rho$

$\mathrm{u}$ : Flow velocity component $(\mathrm{m} / \mathrm{s})$

$\mathrm{t}$ : Time step (s)

$\sigma_{k}$ : Prandtl number governing turbulent diffusion of $\mathrm{k}$

$\sigma_{\varepsilon}$ : Prandtl number governing turbulent diffusion of $\varepsilon$

$\tau_{t i j}:$ Reynolds stress tensor

$\mu$ : Dynamic viscosity $(\mathrm{kg} / \mathrm{ms})$

$C_{1^{\varepsilon}}:$ Empirical constant $=1.44$

$\mathrm{i}, \mathrm{j}, \mathrm{k}, \mathrm{N}$ : General parameters
Q: Volume flow rate of air $\left(\mathrm{m}^{3} / \mathrm{s}\right)$

$\mathrm{r}$ : Entrance duct radius ( $\mathrm{mm}$ )

$\mathrm{R}_{\mathrm{E}}$ : Ellipsoidal hub ratio

p: Static pressure $(\mathrm{Pa})$

$\rho$ : Density of air $\left(\mathrm{kg} / \mathrm{m}^{3}\right)$

$\mathrm{r}_{\mathrm{h}}$ : Hub radius $(\mathrm{mm})$

$\phi:$ Volume coefficient

$1_{\mathrm{h}}$ : Axial hub length (mm)

$\varepsilon_{h}:$ Hub effectiveness

$\zeta$ : Static pressure rise coefficient

TVR: Turbulent viscosity ratio

v: Grid velocity component $(\mathrm{m} / \mathrm{s})$

$\mathrm{k}$ : Turbulent kinetic energy $(\mathrm{J} / \mathrm{kg})$

$\varepsilon$ : Rate of turbulent dissipation

$G_{k}$ : Production of k due to mean velocity gradients

$S_{u i}:$ Source term for $\mathrm{u}$ equation

$\tau_{i j}$ : Molecular stress tensor for Newtonian

fluids

$C_{2^{\varepsilon}}:$ Empirical constant $=1.92$

\section{Subscripts}

1: Fan inlet

2: Impeller exit

3: Diffuser exit 


\section{Declaration}

- Availability of data and materials:

The datasets used and/or analyzed during the current study are available from the corresponding author on reasonable request.

* Competing interests:

The authors declare that they have no competing interests

\section{Funding}

There is no funding for the study presented in this article.

\section{* Authors' contributions}

MN (First author) performed numerical analysis and captured data.

KVK (Second author) helped to capture data and plots, monitored progress of the work

$\square$ NYS (Corresponding author) explored literature, identified research gap, guidance for work, manuscript preparation

All authors read and approved the final manuscript

\section{Acknowledgements}

The computational facility for conducting this research work was provided by the Department of Mechanical and Manufacturing Engineering, Manipal Institute of Technology, Manipal Academy of Higher Education (MAHE), Manipal. The authors wish to express their gratitude and acknowledge the support provided by the institute in carrying out this research work.

\section{References}

[1] Vagnoli S. and Verstraete, T., 2015, "URANS analysis of the effect of realistic inlet distortions on the stall inception of a centrifugal compressor", Computers and Fluids. Elsevier Ltd, 116, pp. 192-204. doi: 10.1016/j.compfluid.2015.03.015.

[2] Wang Y., Dong, Q. and Wang, P., 2015, "Numerical investigation on fluid flow in a 90degree curved pipe with large curvature ratio", Mathematical Problems in Engineering, 2015. doi: 10.1155/2015/548262.

[3] Gholamian M., Rao, G. K. M. and Panitapu, B., 2013, "Effect of axial gap between inlet nozzle and impeller on efficiency and flow pattern in centrifugal fans, numerical and 
experimental analysis", Case Studies in Thermal Engineering. Elsevier, 1(1), pp. 26-37. doi: 10.1016/j.csite.2013.08.003.

[4] Son P. N., Kim, J. and Ahn, E. Y., 2011, "Effects of bell mouth geometries on the flow rate of centrifugal blowers", Journal of Mechanical Science and Technology, 25(9), pp. 2267-2276. doi: 10.1007/s12206-011-0609-3.

[5] Yang C, Chen Shan, Li Du, Yang Changmao, and Wang Yidi., 2010, "Inlet Recirculation Influence to the Flow Structure of Centrifugal Impeller", Chinese Journal of Mechanical Engineering, 23(05), p. 647. doi: 10.3901/CJME.2010.05.647.

[6] Cai, R., 1999, “The Influence of Shrouded Stator Cavity Flows on Multistage Compressor Performance", ASME J. Turbomach., 121(3), p. 497.

[7] Meakhail T and Park, S. O., 2005, "A Study of Impeller-Diffuser-Volute Interaction in a Centrifugal Fan”, Volume 5: Turbo Expo 2004, Parts A and B, 127(January 2005), pp. 687-695. doi: 10.1115/GT2004-53068.

[8] K Vasudeva Karanth and N. Yagnesh Sharma., 2009, "Numerical Analysis of a Centrifugal Fan for Performance Enhancement using Boundary Layer Suction Slots", Proc. IMechE Vol. 224 Part C: J. Mechanical Engineering Science, pp 1665 - 1678.

[9] S K Som and N. Y. Sharma, 2002, "Energy and Exergy Balance in the Process of Spray Combustion in a Gas Turbine Combustor', Journal of Heat Transfer Transactions of ASME, Vol 124 , pp $828-836$.

[10] Sukhmeet Singh, Subhash Chander, J.S. Saini, 2011, "Heat transfer and friction factor correlations of solar air heater ducts artificially roughened with discrete V-down ribs", Energy, Elsevier, Vol 36, pp.5053-5064, DOI: 10.1016/j.energy.2011.05.052. 
Figures
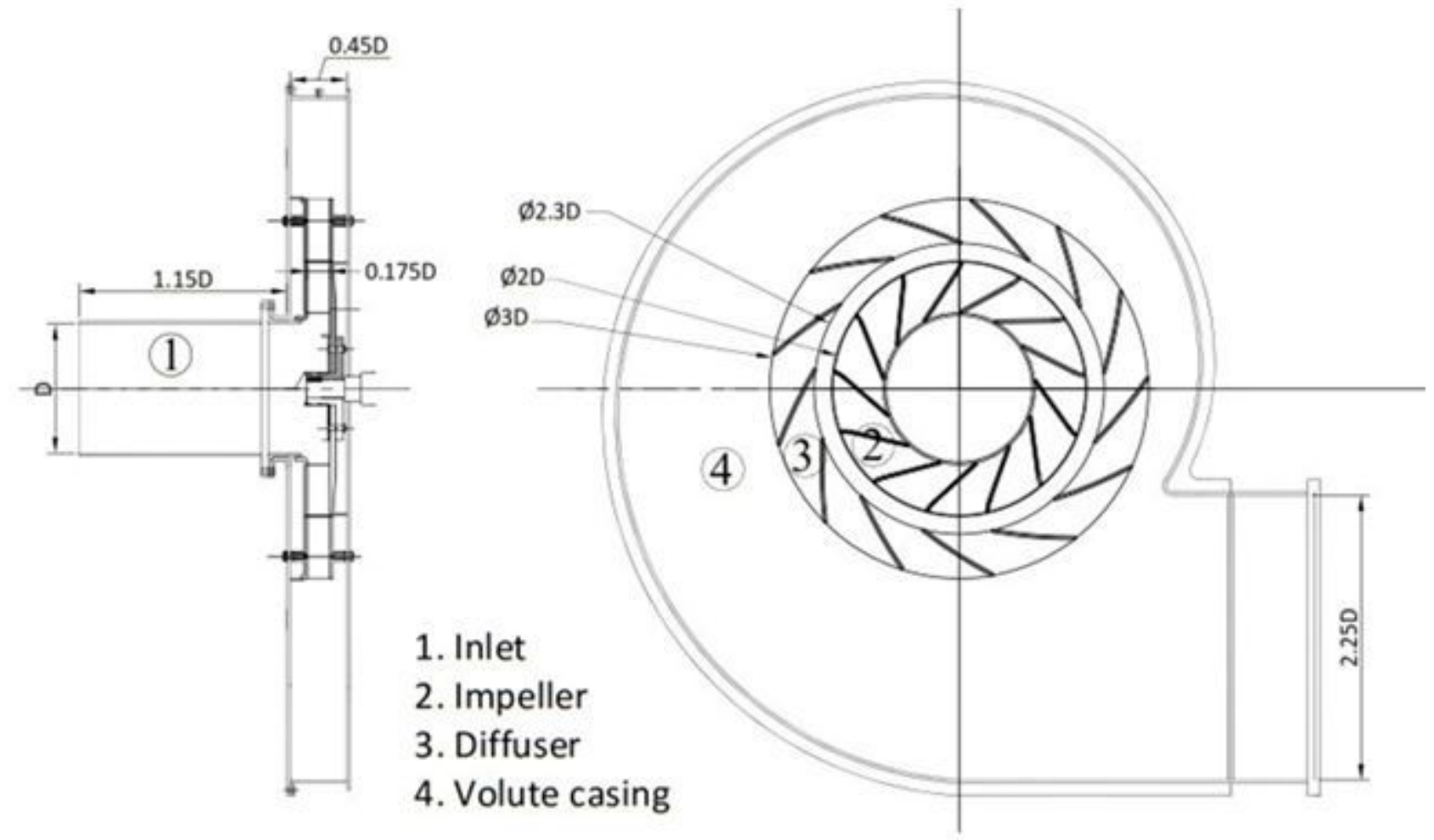

Figure 1

Schematic drawing of centrifugal fan 


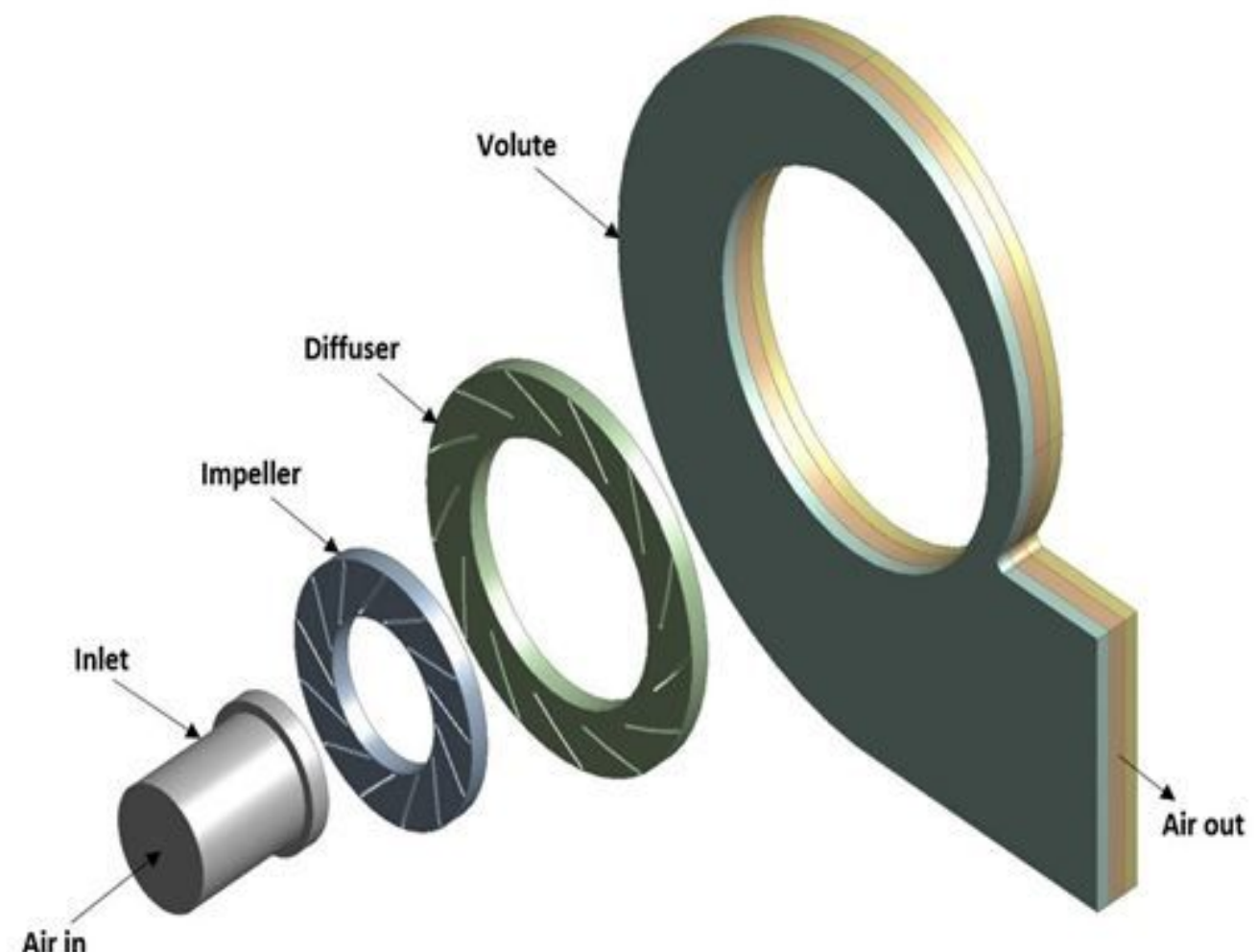

Figure 2

Computational domain of hub-less base configuration considered for the analysis

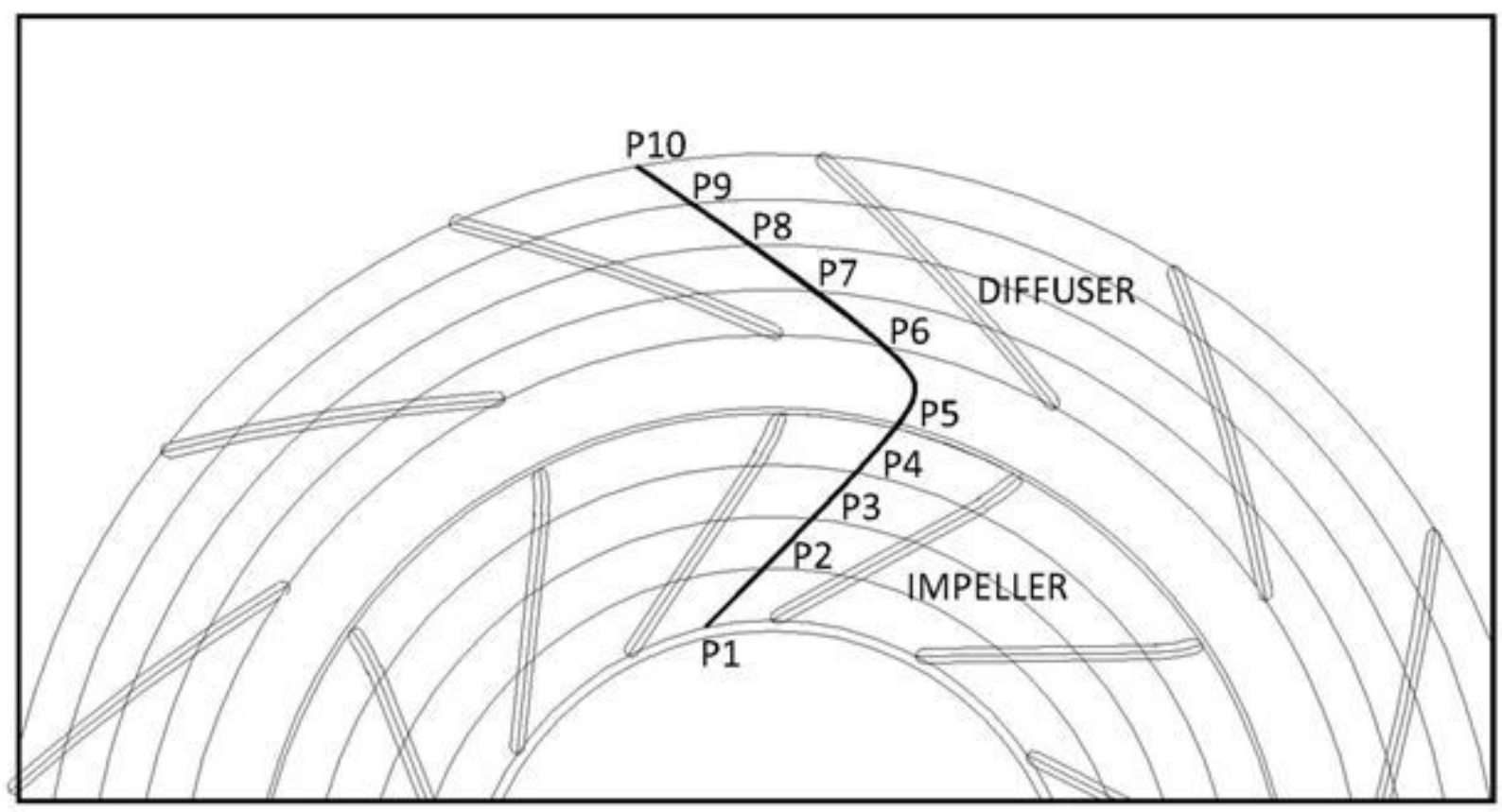

Figure 3

Salient points chosen for mesh sensitivity analysis 


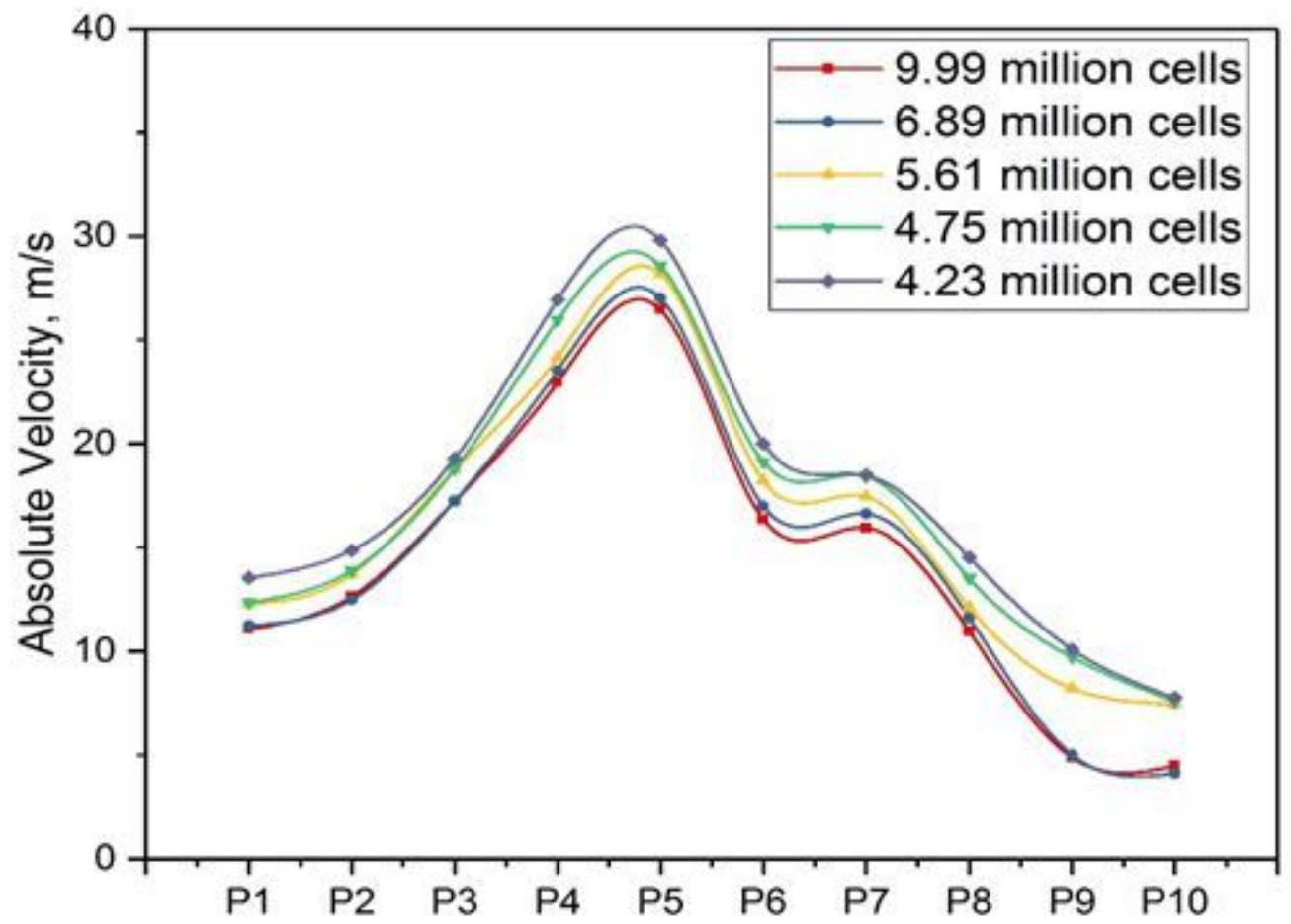

Flow along the Impeller and Diffuser passages

Figure 4

Mesh sensitivity test results based on absolute velocity

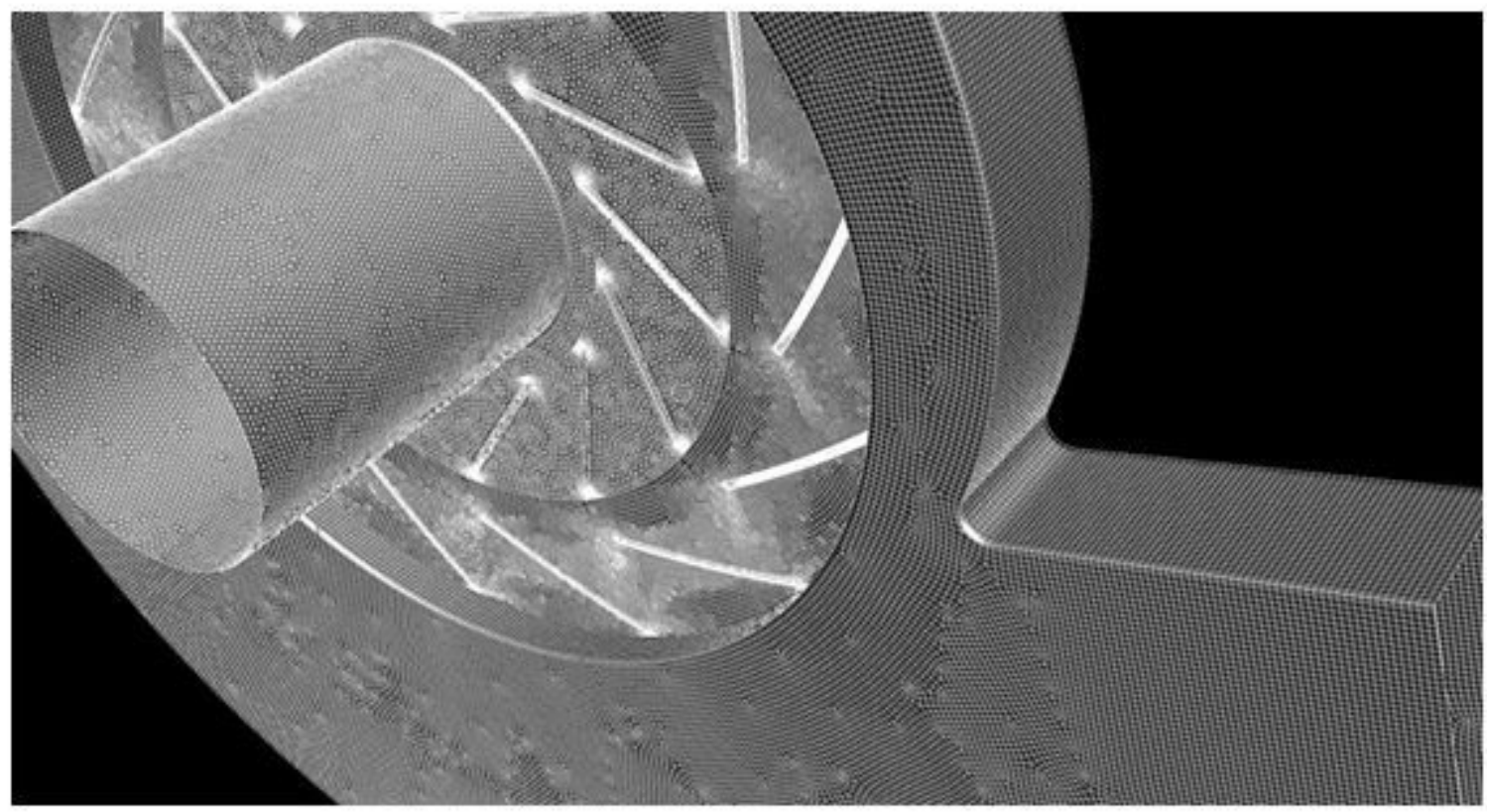

Figure 5

A magnified view of meshed flow regions 


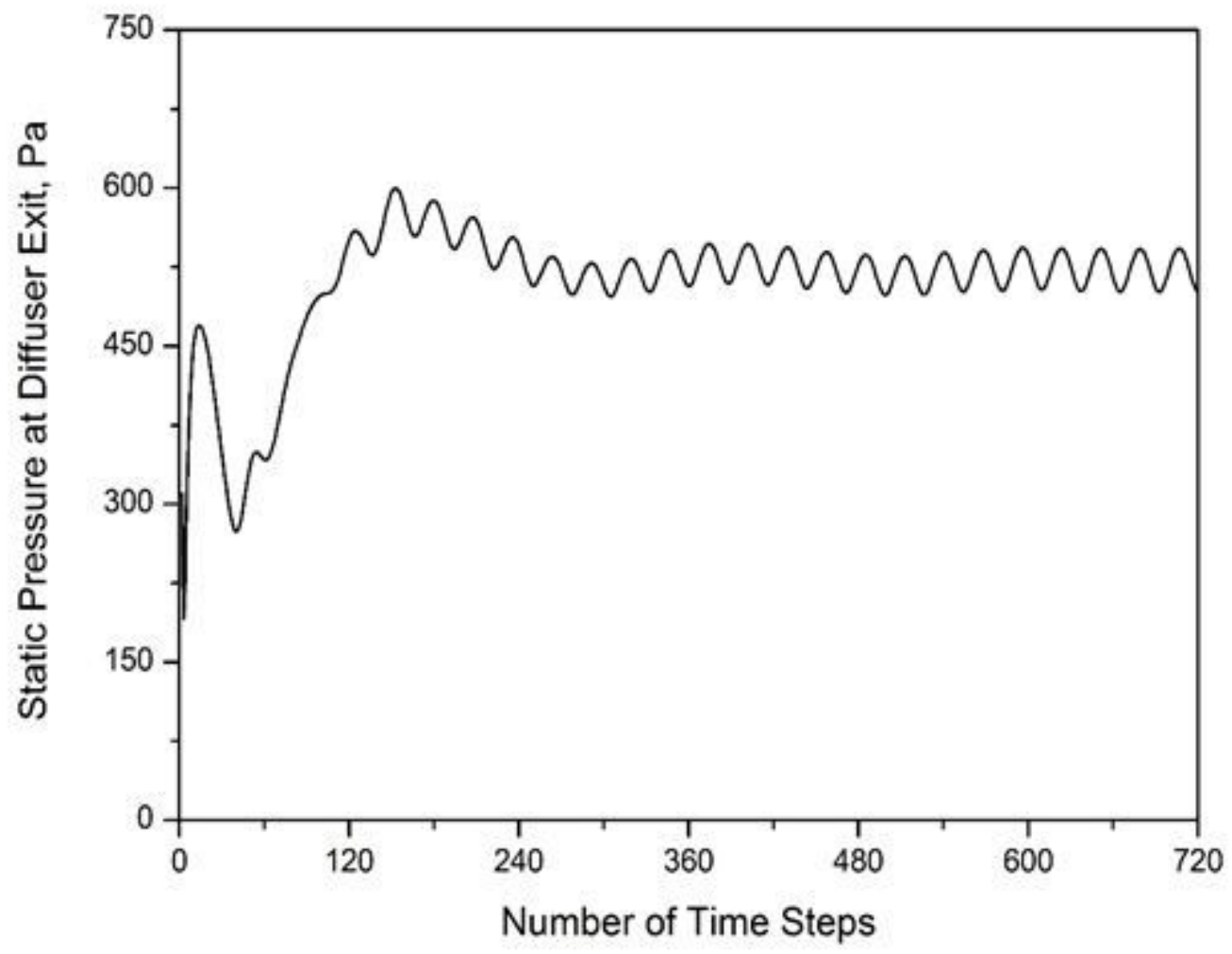

Figure 6

Pressure fluctuation graph captured at the exit of the diffuser up to two rotations of the impeller

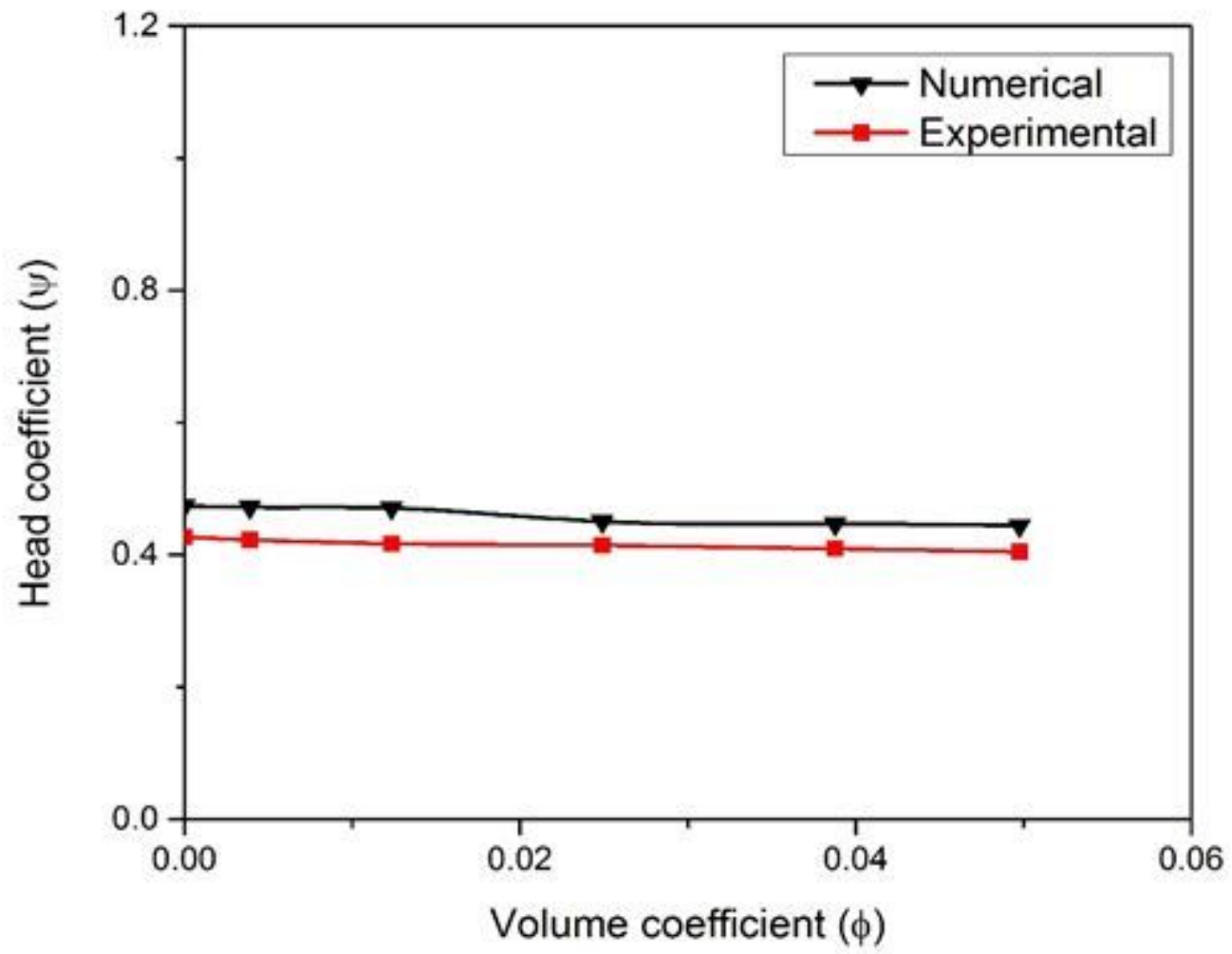

Figure 7 
The main characteristics for validation study

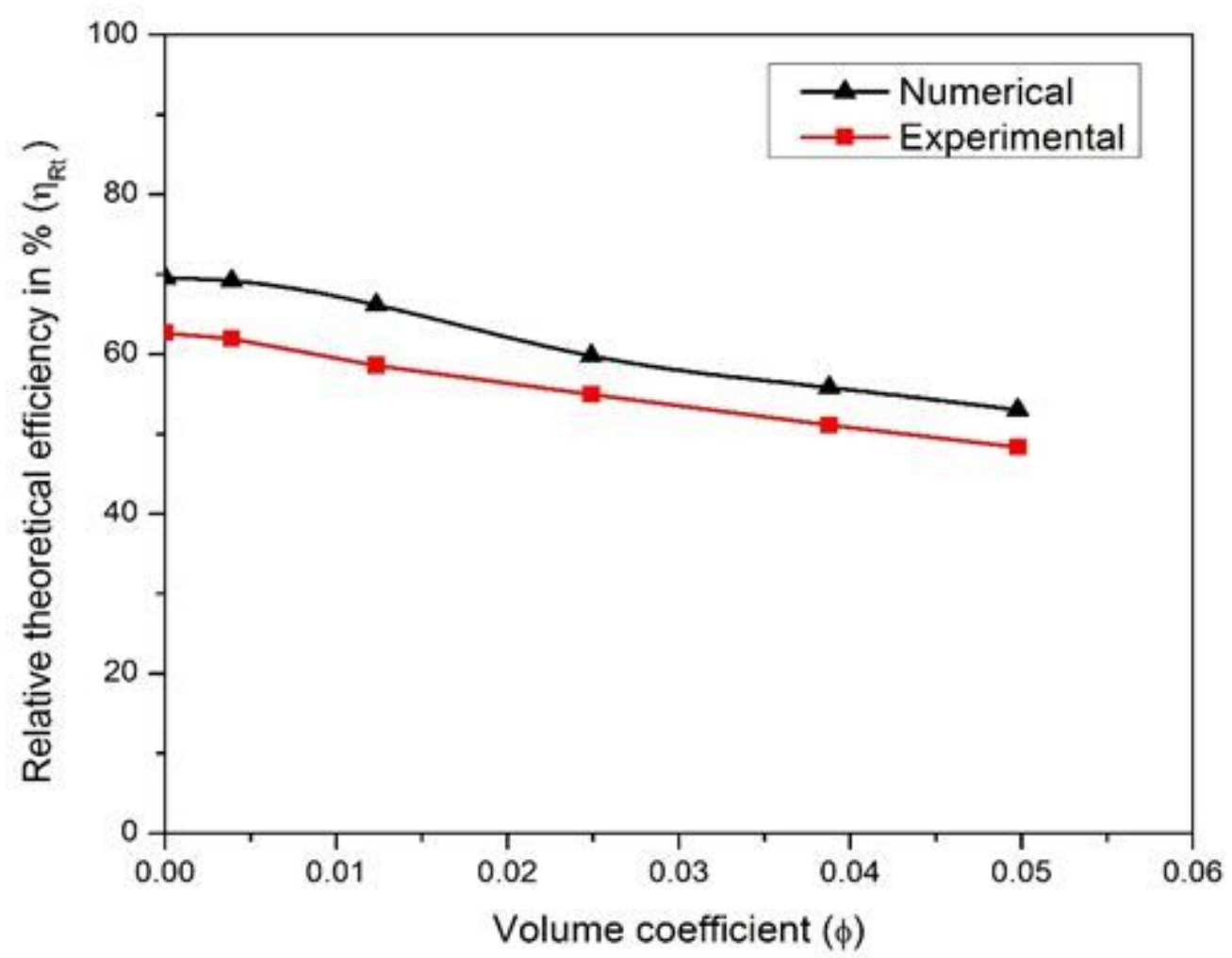

Figure 8

The operating characteristics for validation study

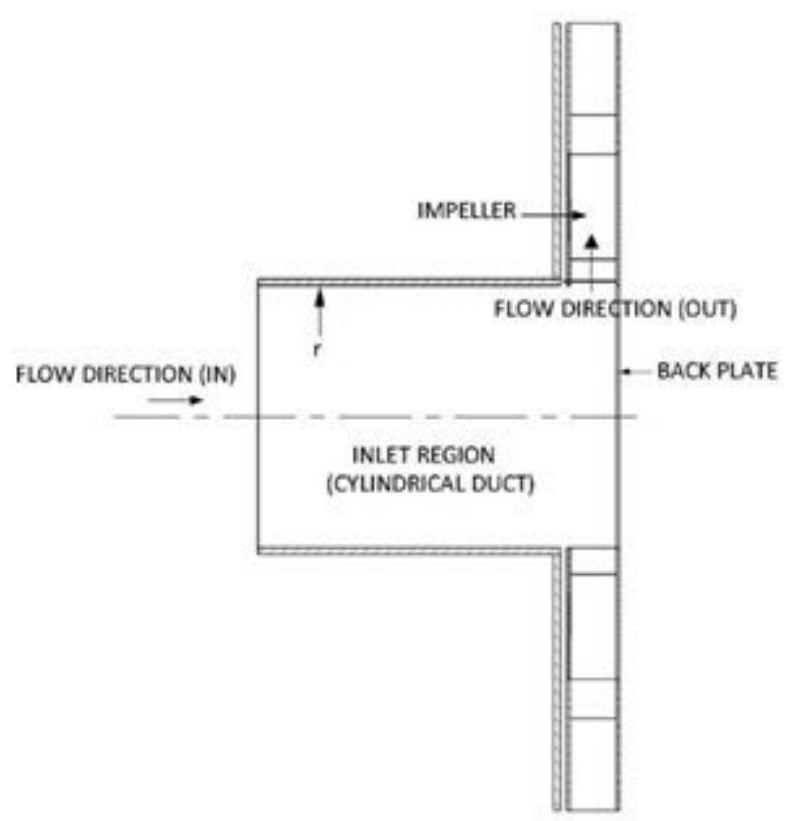

Figure $9(A)$

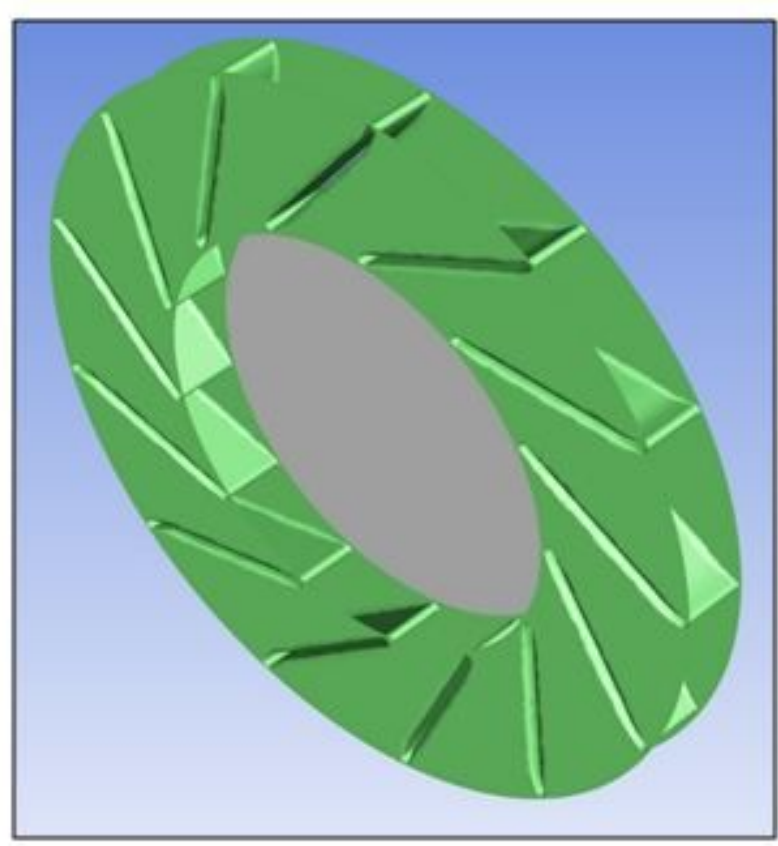

Figure 9 (B)

Figure 9 
Geometric configuration for the hub-less configuration

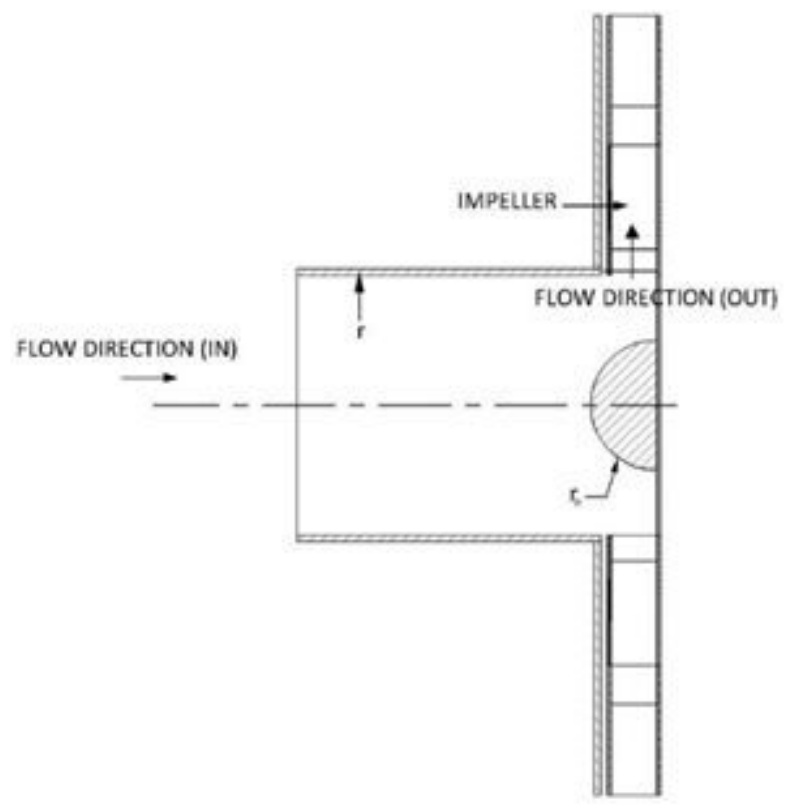

Figure $10(\mathrm{~A})$

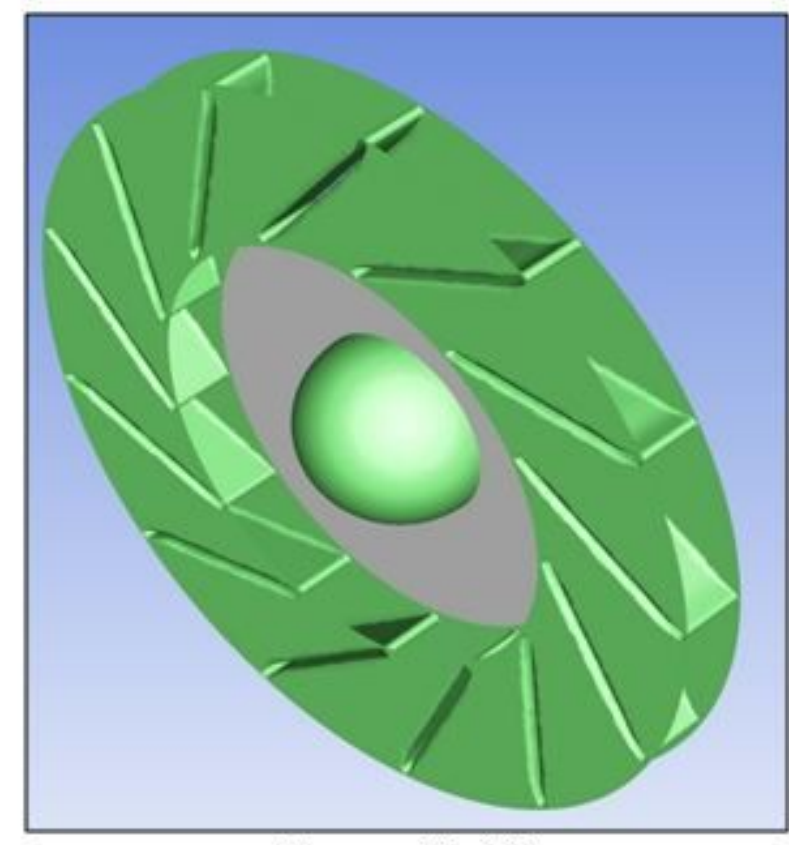

Figure $10(\mathrm{~B})$

\section{Figure 10}

Geometric configuration for the hemi-spherical hub configuration

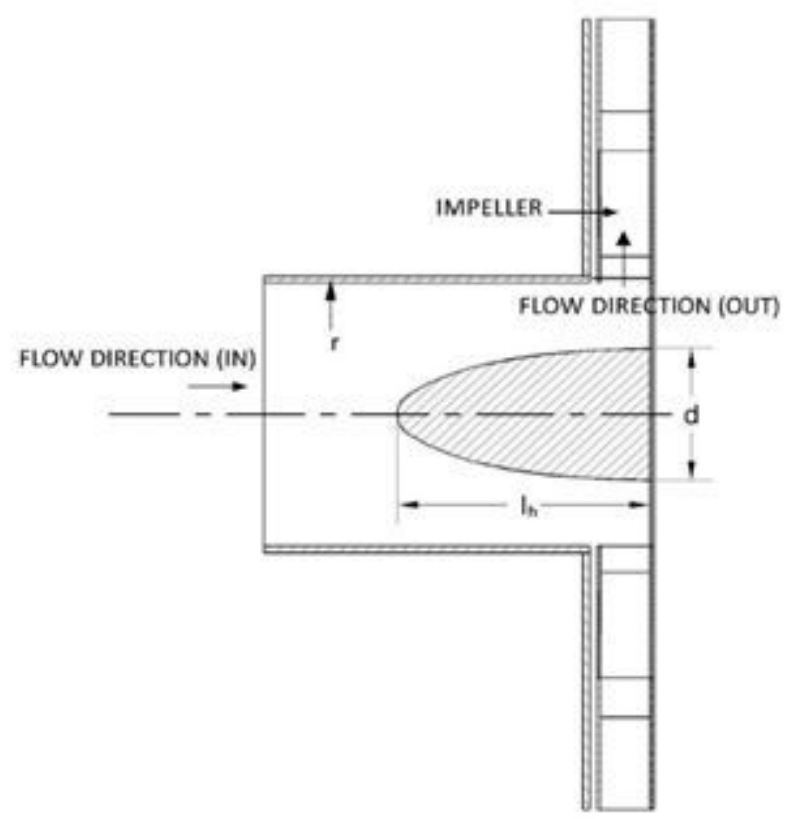

Figure $11(A)$

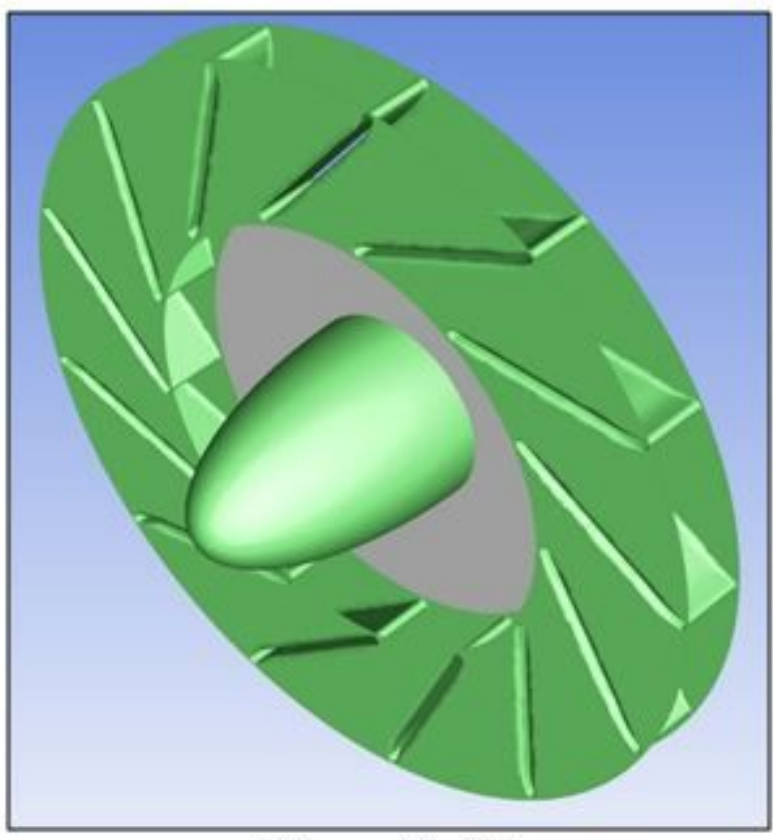

Figure $11(\mathrm{~B})$

\section{Figure 11}

Geometric configuration for the ellipsoidal hub configuration 


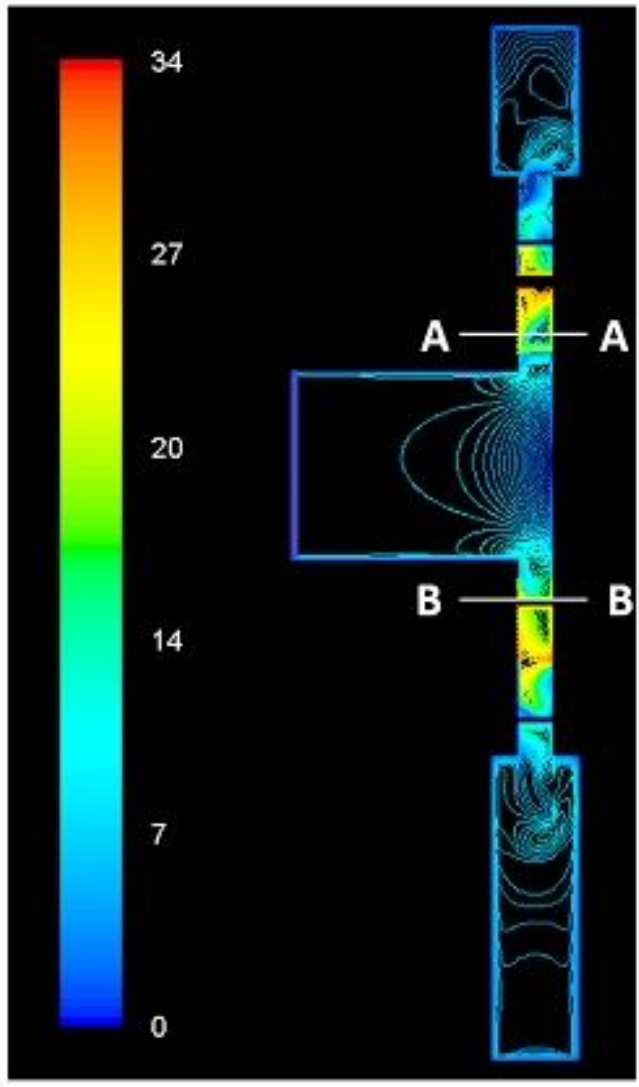

Figure 12(A): Profile view

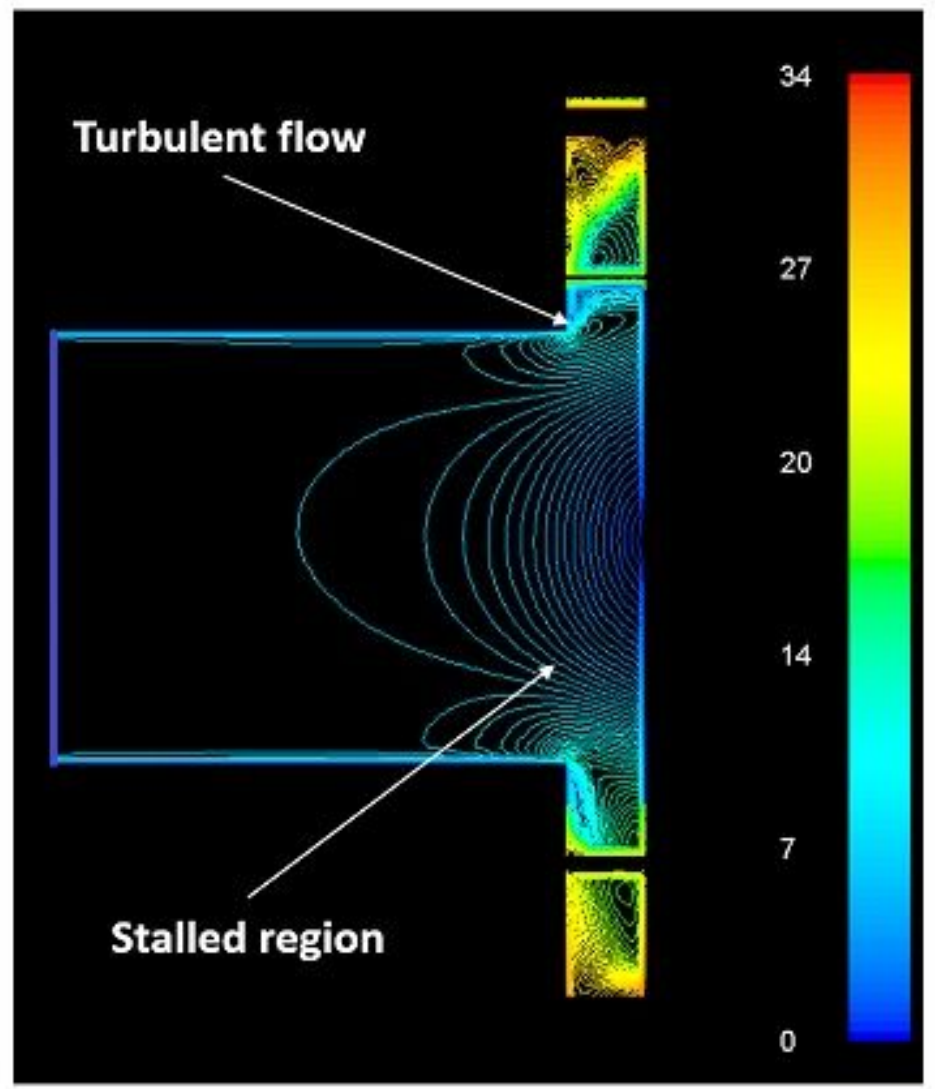

Figure 12(B): Magnified view across Section AA $\mathrm{BB}$

Figure 12

Velocity contour plot for the hub-less base configuration 


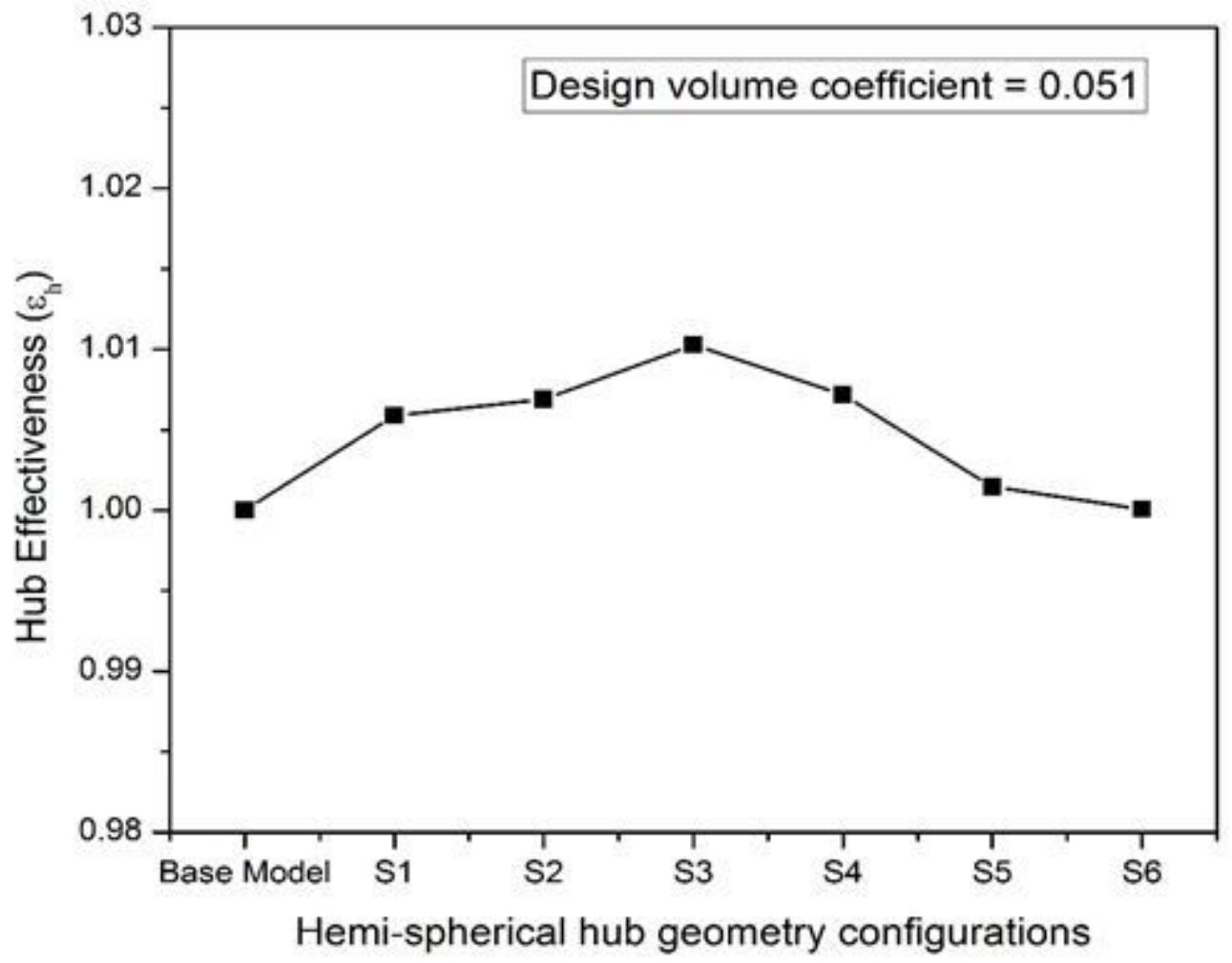

Figure 13

Hub effectiveness for various hemi-spherical hub geometry configurations

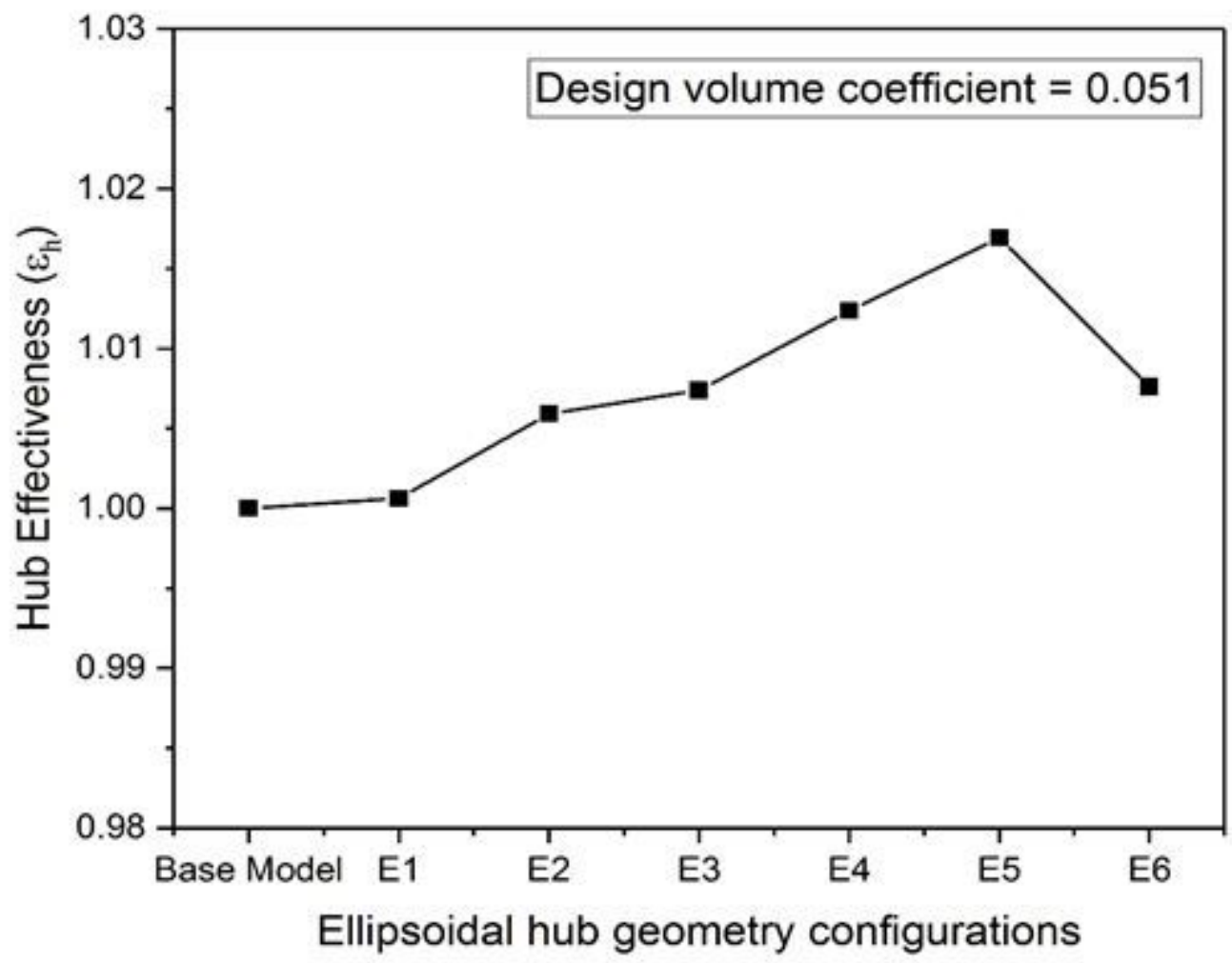

Figure 14 
Hub effectiveness for various ellipsoidal hub geometry configurations

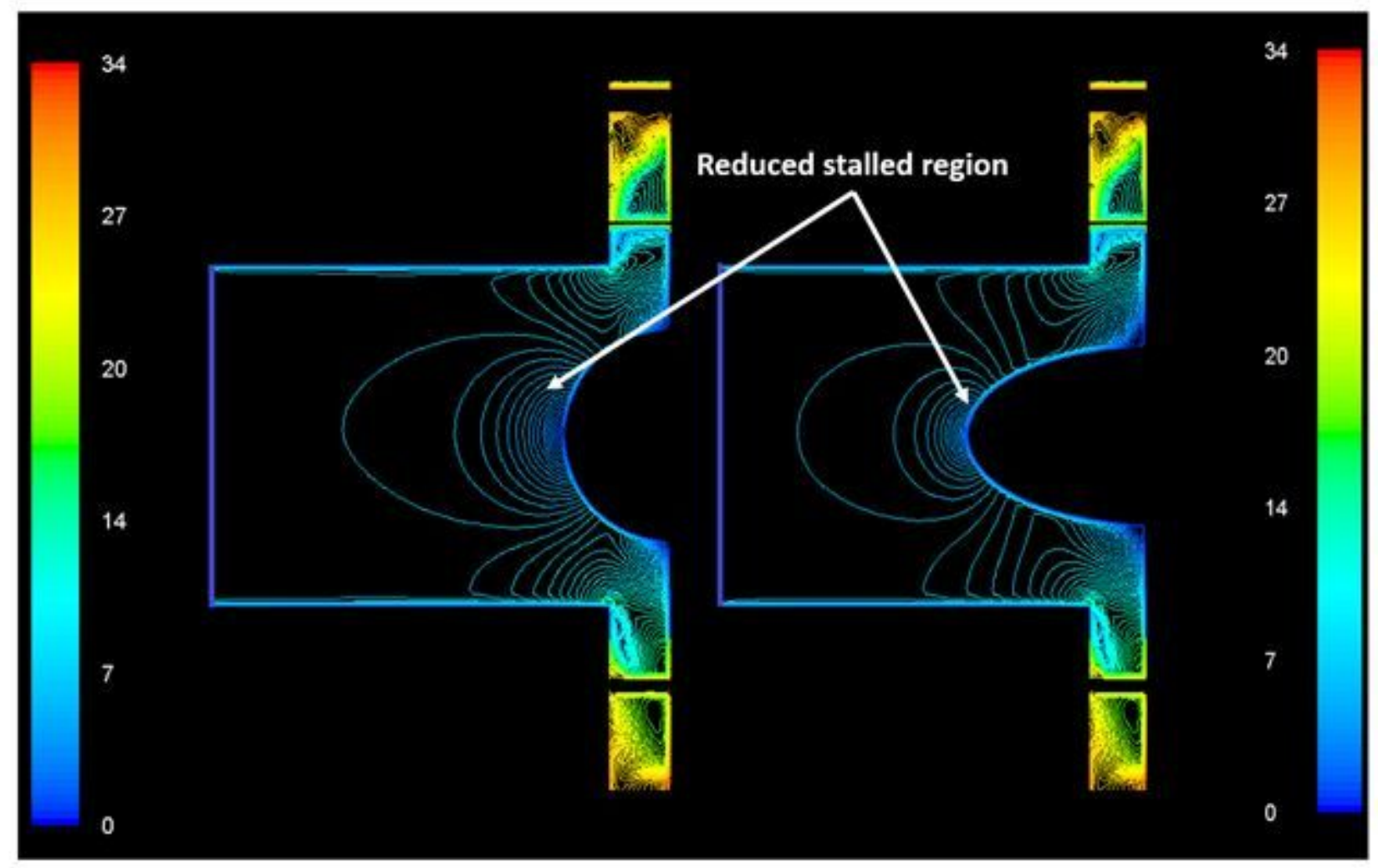

Figure 15 (A): Configuration S3 $\left(\mathrm{R}_{\mathrm{s}}=0.5\right)$

Figure 15 (B): Configuration E5 $\left(\mathrm{R}_{\mathrm{E}}=1.1\right)$

\section{Figure 15}

Velocity contour plots for optimized hub geometry configurations 


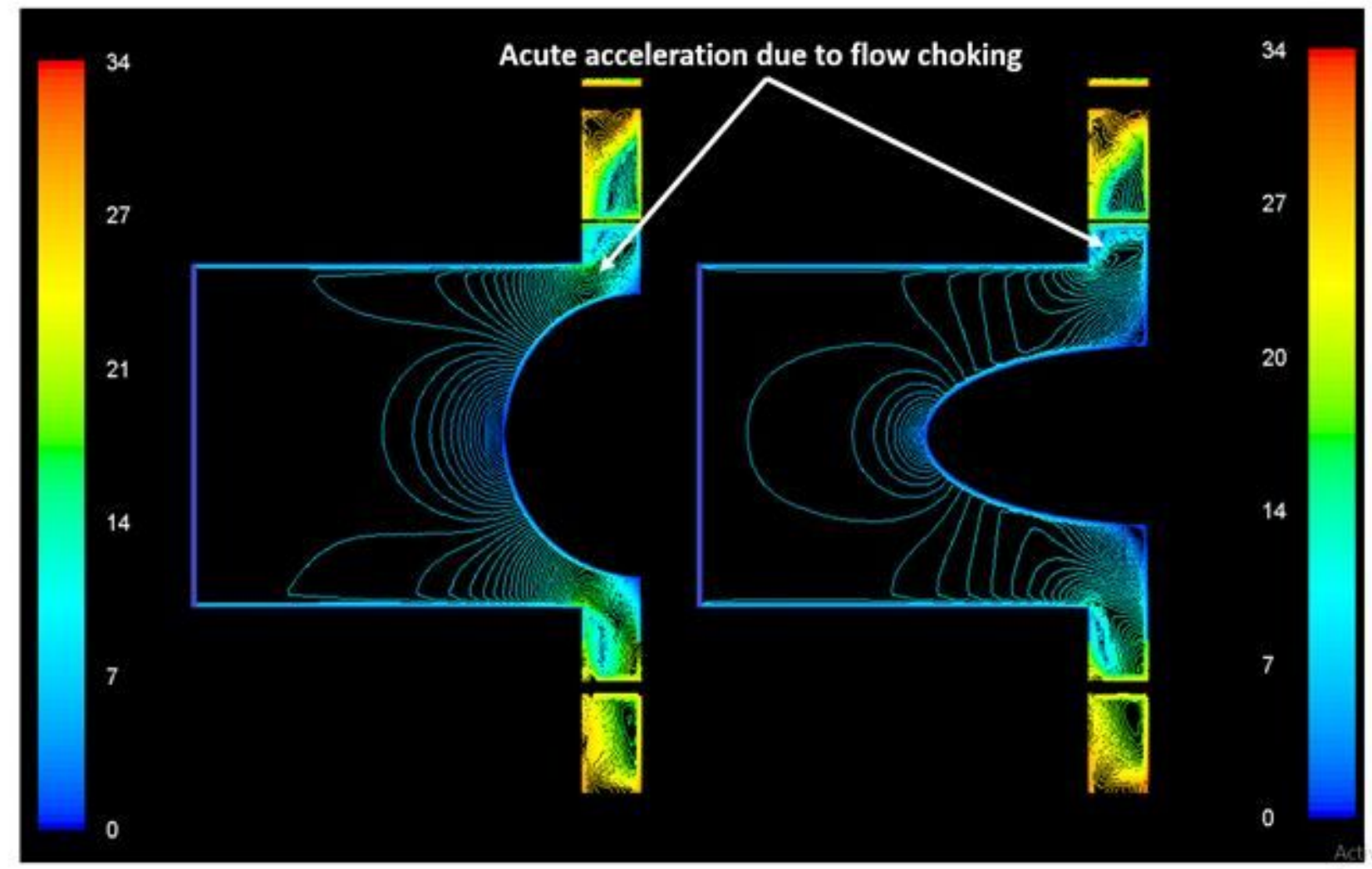

Figure 16(A): Configuration S6

$$
\left(\mathrm{R}_{\mathrm{s}}=0.8\right)
$$

Figure 16(B): Configuration E6 $\left(\mathrm{R}_{\mathrm{E}}=1.3\right)$

Figure 16

Velocity contour plots for various hub geometry configurations 


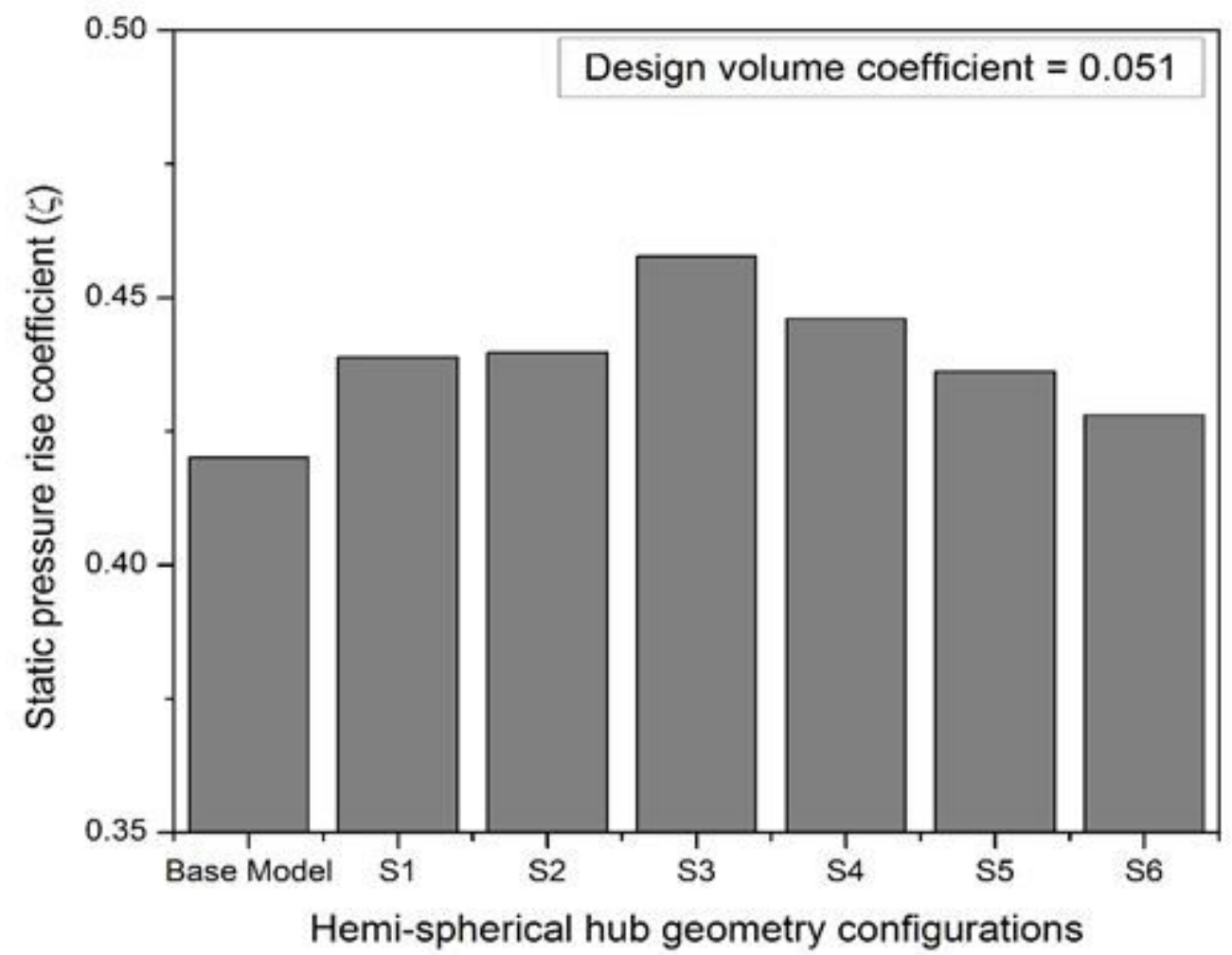

Figure 17

Static pressure rise coefficient for various hemi-spherical hub geometry configurations

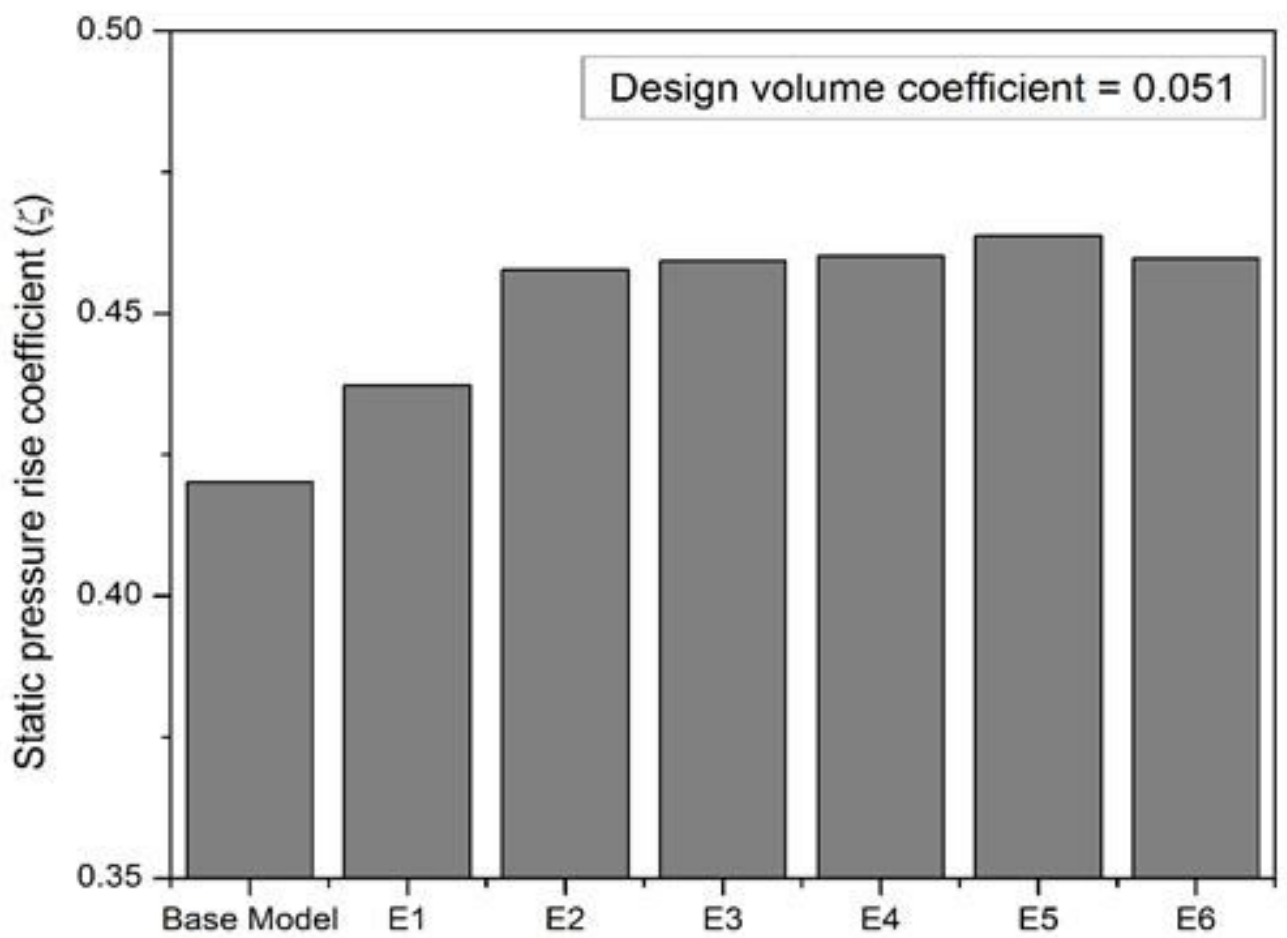

Ellipsoidal hub geometry configurations

Figure 18 
Static pressure rise coefficient for various ellipsoidal hub geometry configurations

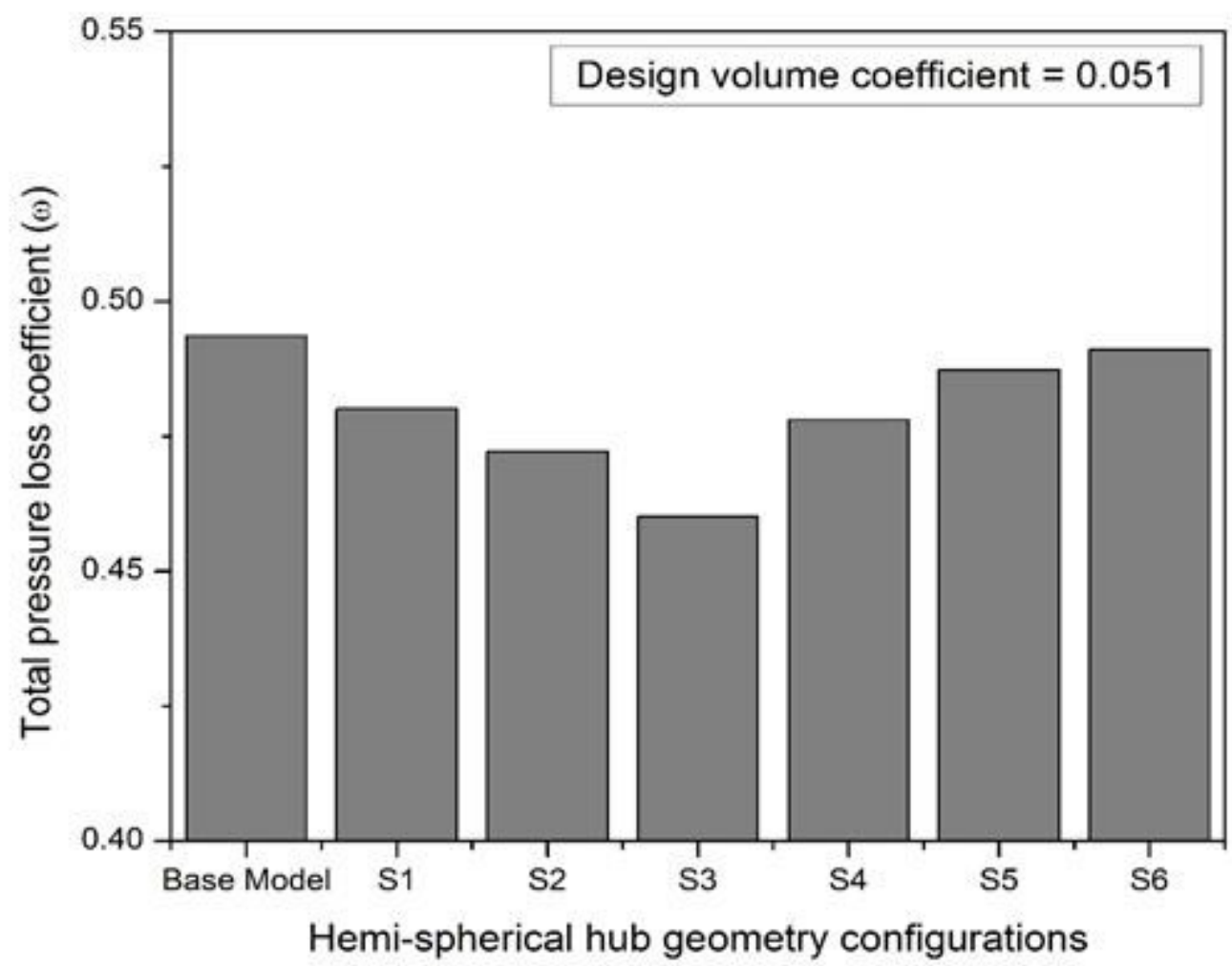

Figure 19

Total pressure loss coefficient for various hemi-spherical hub geometry configurations

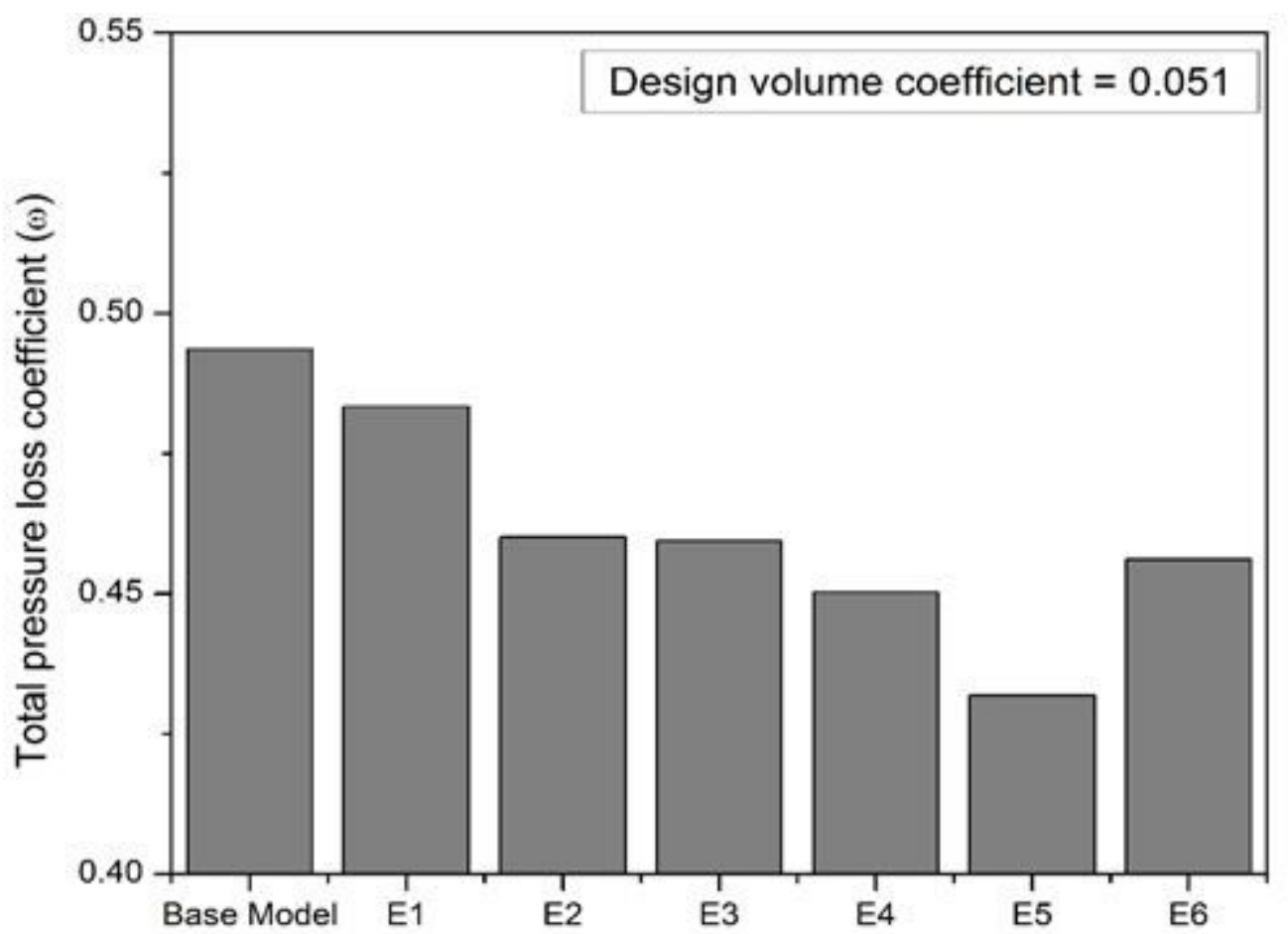

Ellipsoidal hub geometry configurations

Figure 20 
Total pressure loss coefficient for various ellipsoidal hub geometry configurations

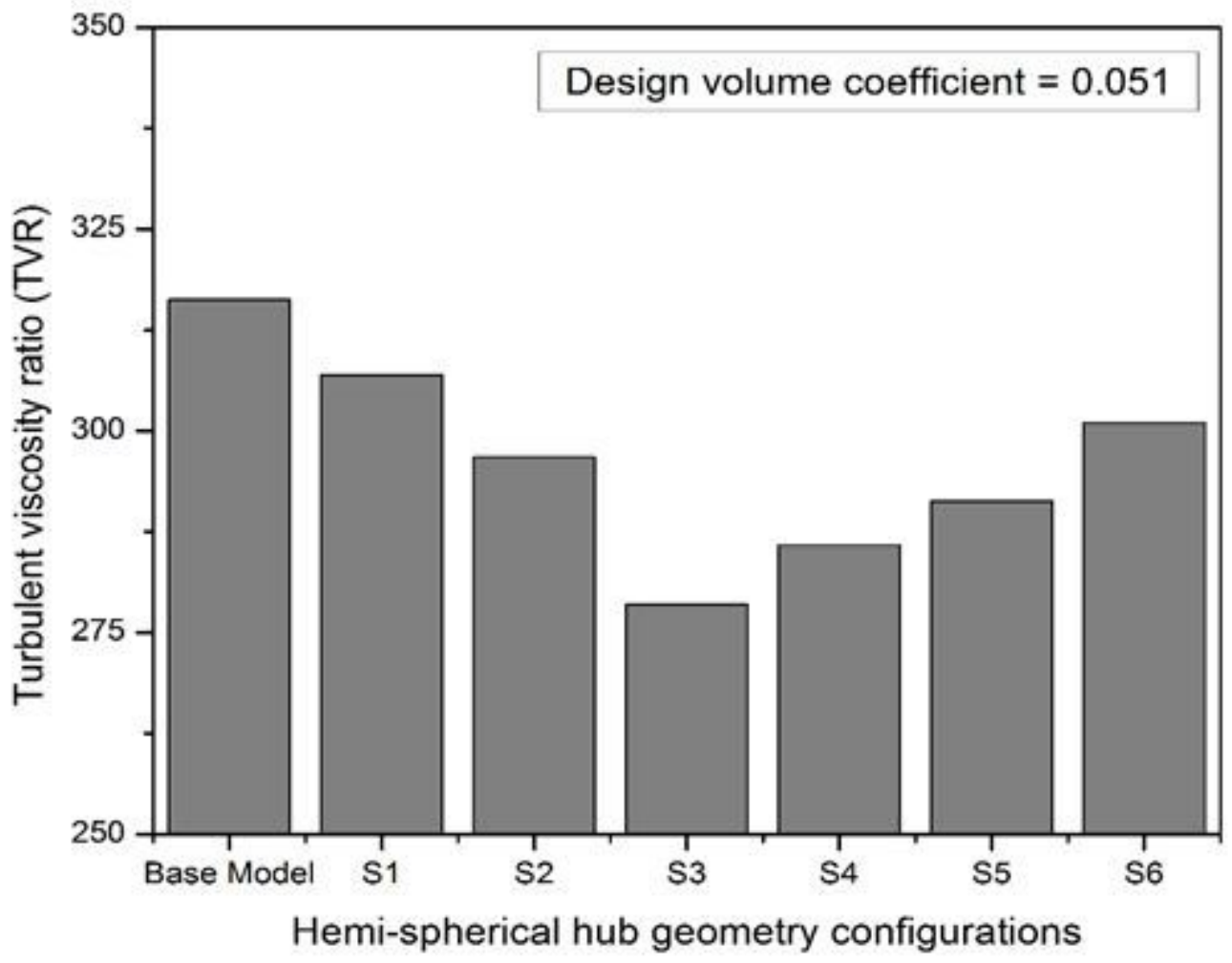

Figure 21

Turbulent viscosity ratio for various hemi-spherical hub geometry configurations

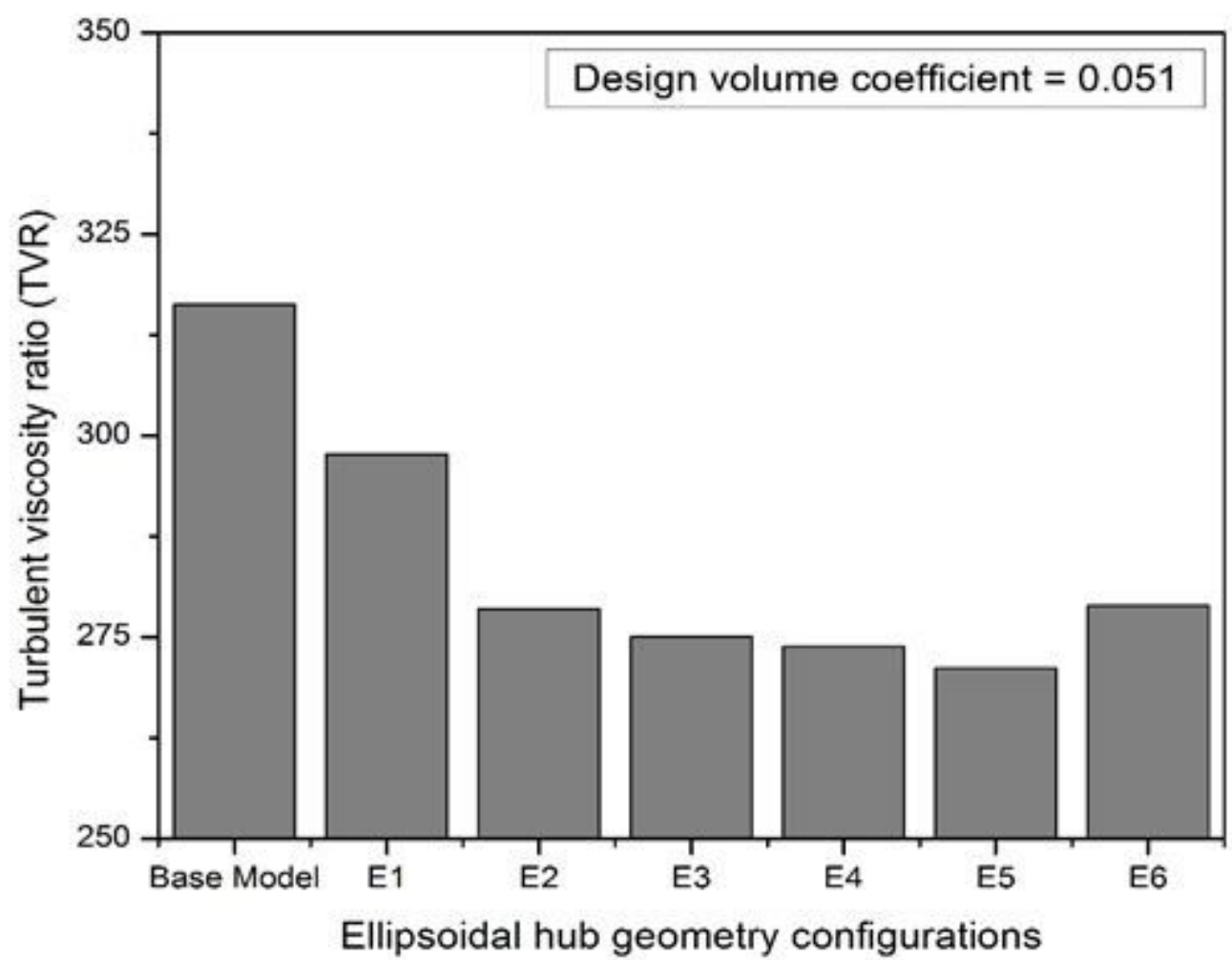

Figure 22 


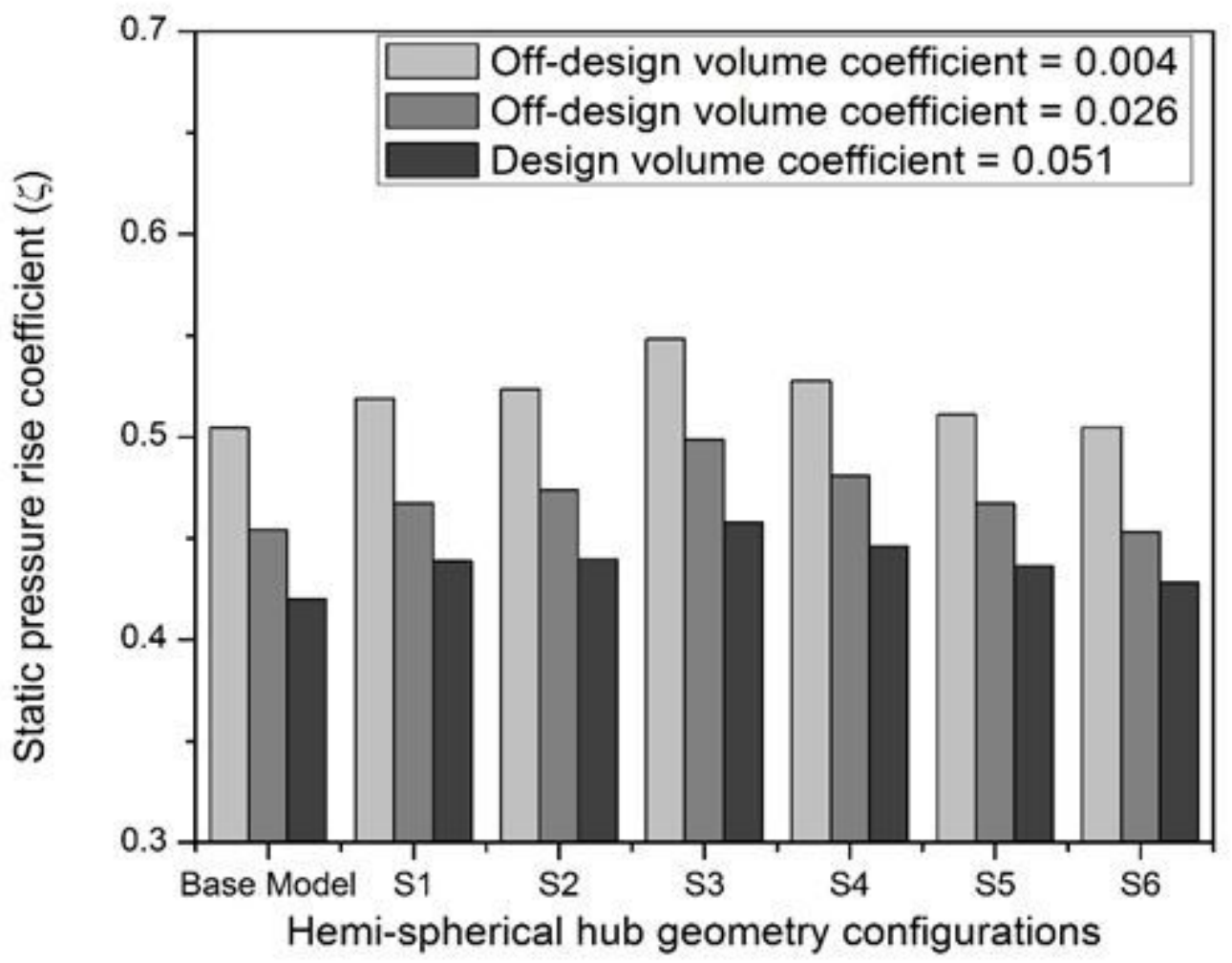

Figure 23

Static pressure rise coefficient of hemi-spherical hub geometry configurations for various volume coefficients

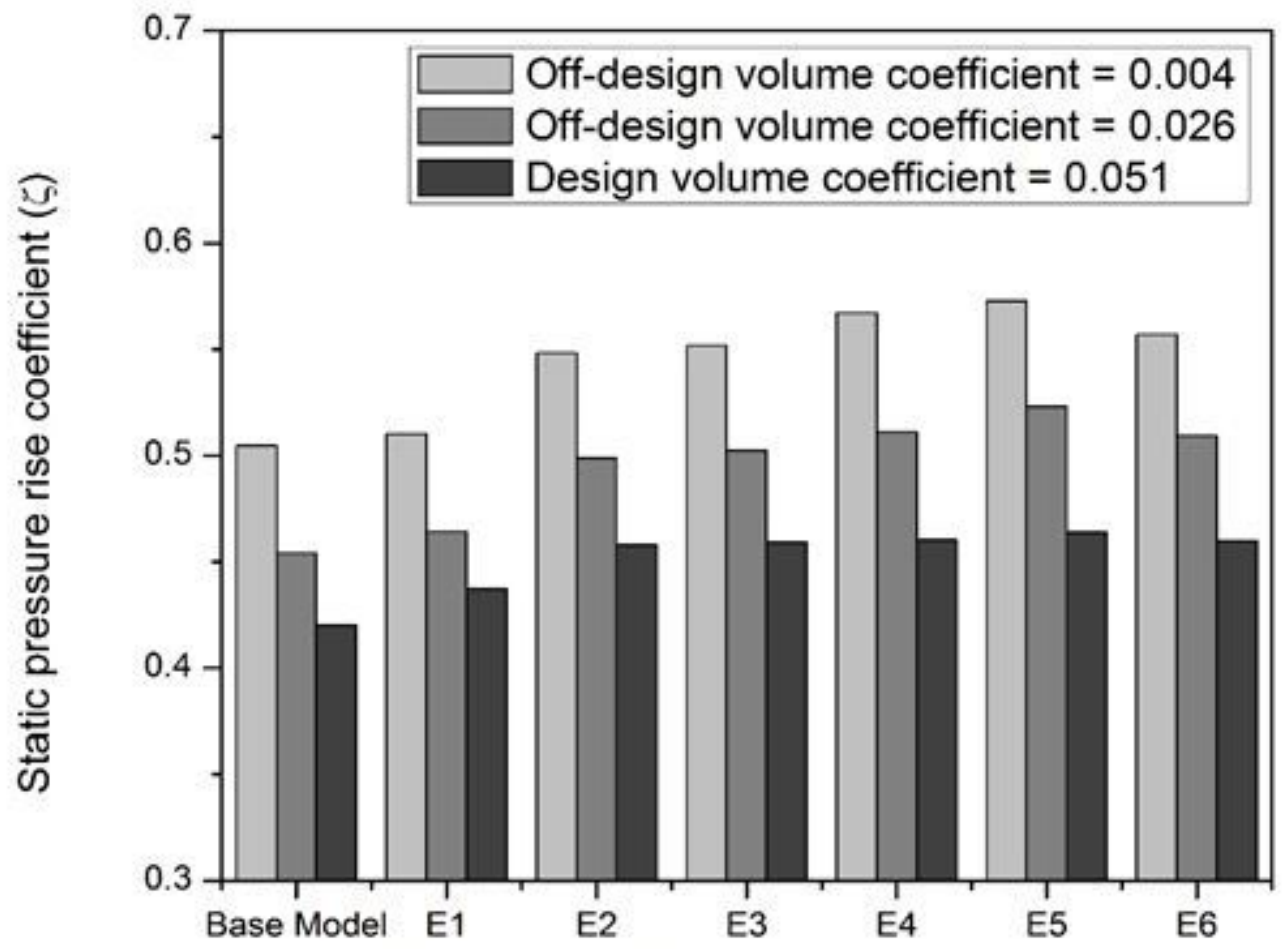

Ellipsoidal hub geometry configurations 
Figure 24

Static pressure rise coefficient of ellipsoidal hub geometry configurations for various volume coefficients

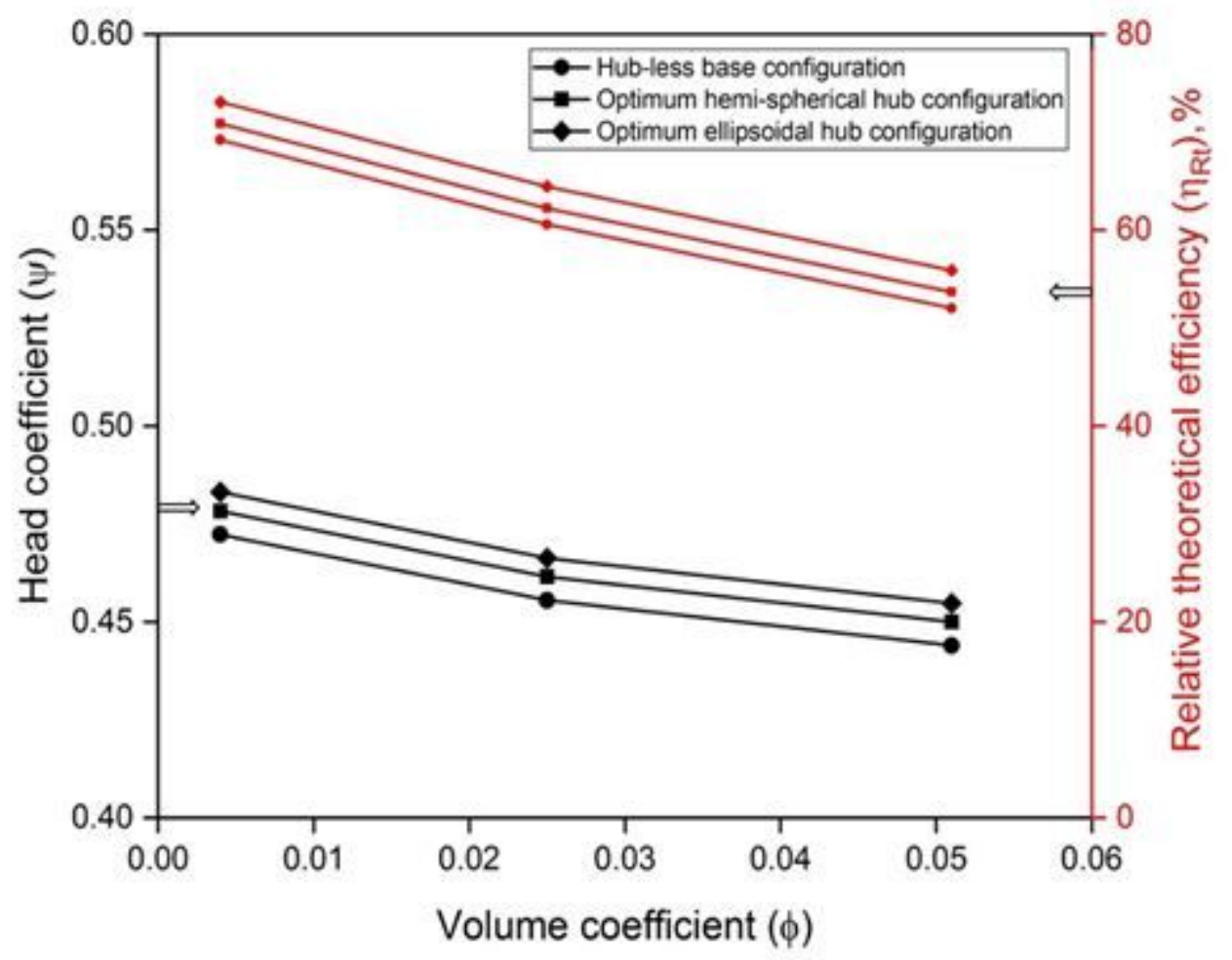

Figure 25

Performance comparison plots for optimized hemi-spherical and ellipsoidal hub configurations

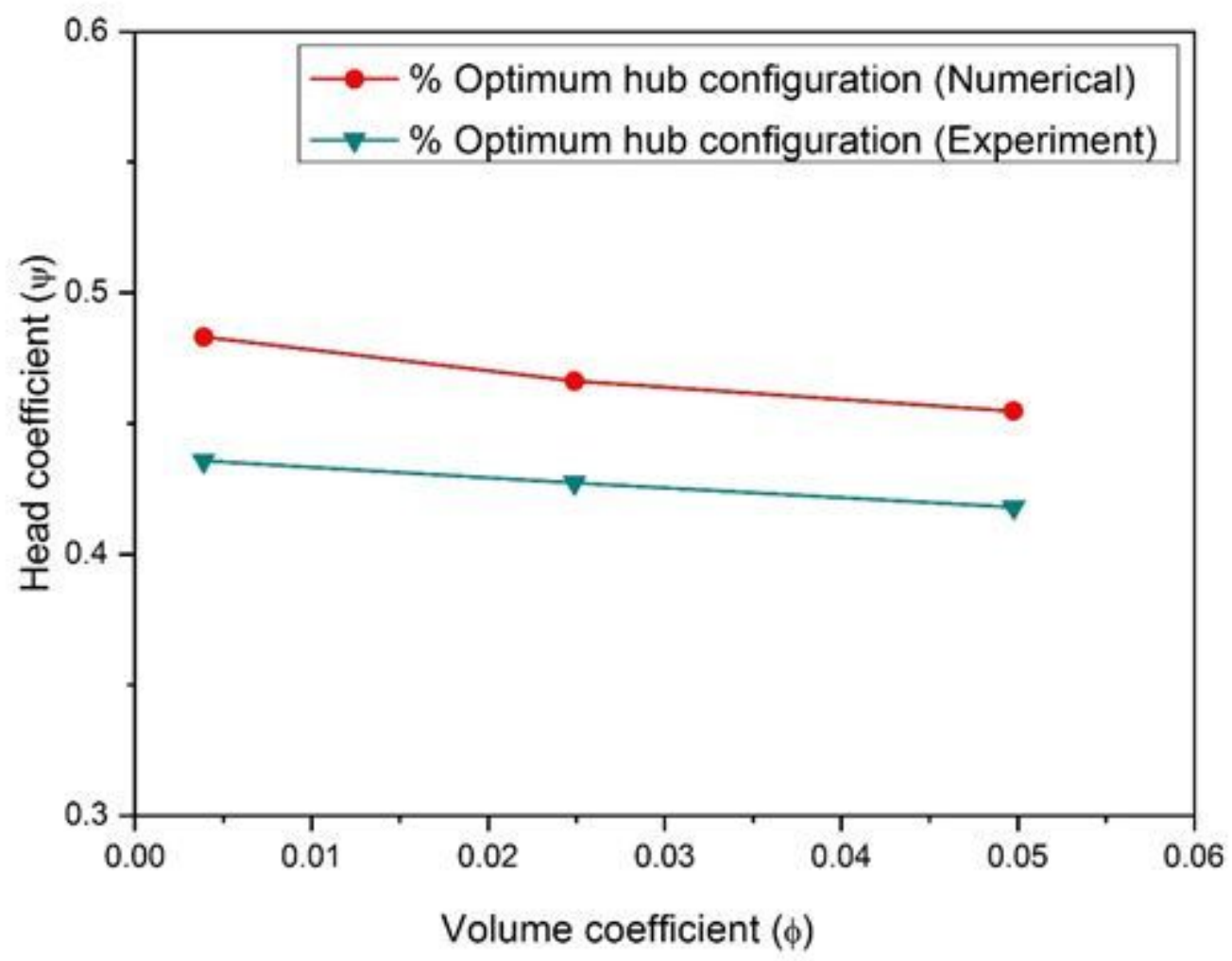


Figure 26

Comparison of head coefficient for the optimum hub geometry configuration

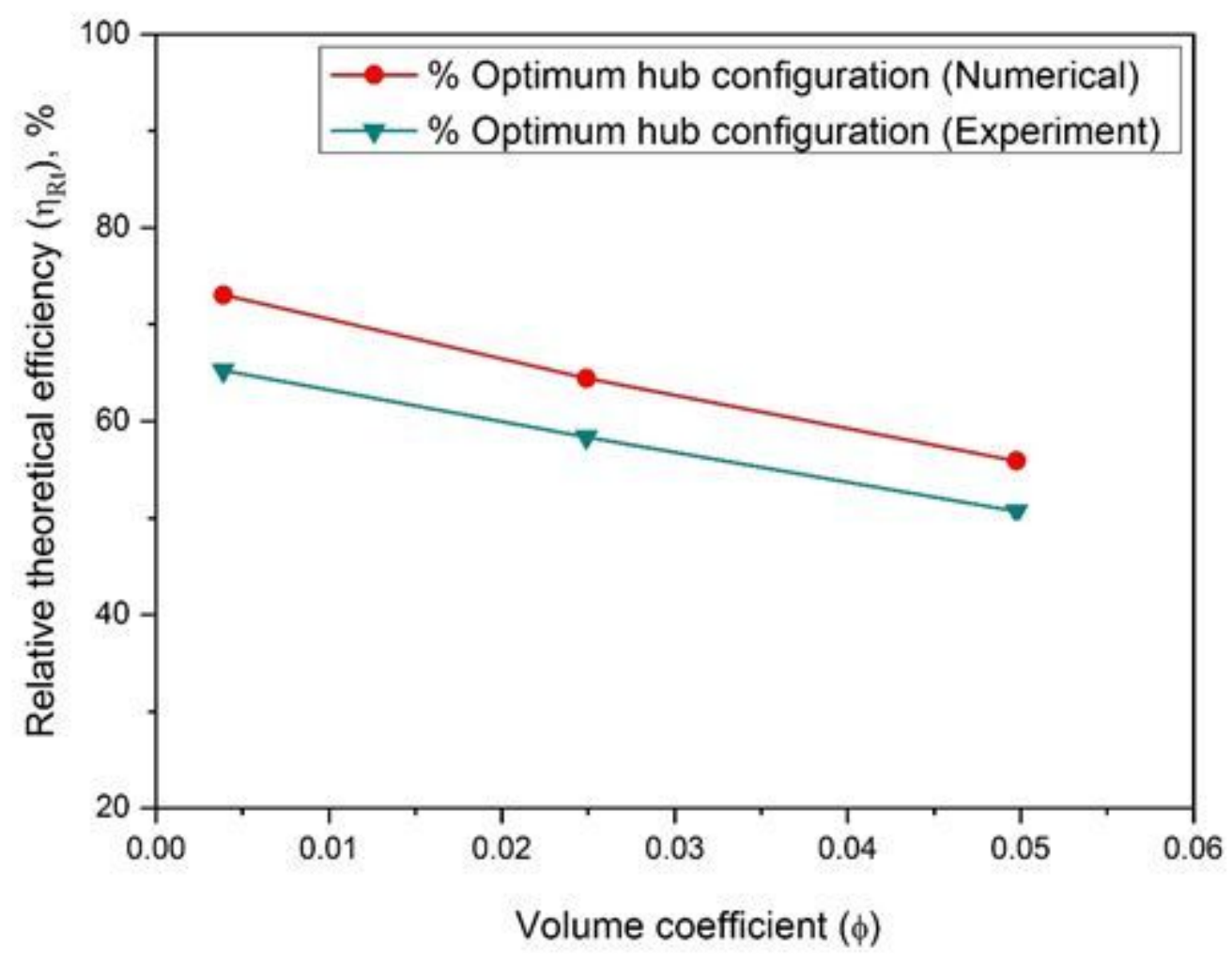

Figure 27

Comparison of relative theoretical efficiency for the optimum hub geometry configuration 


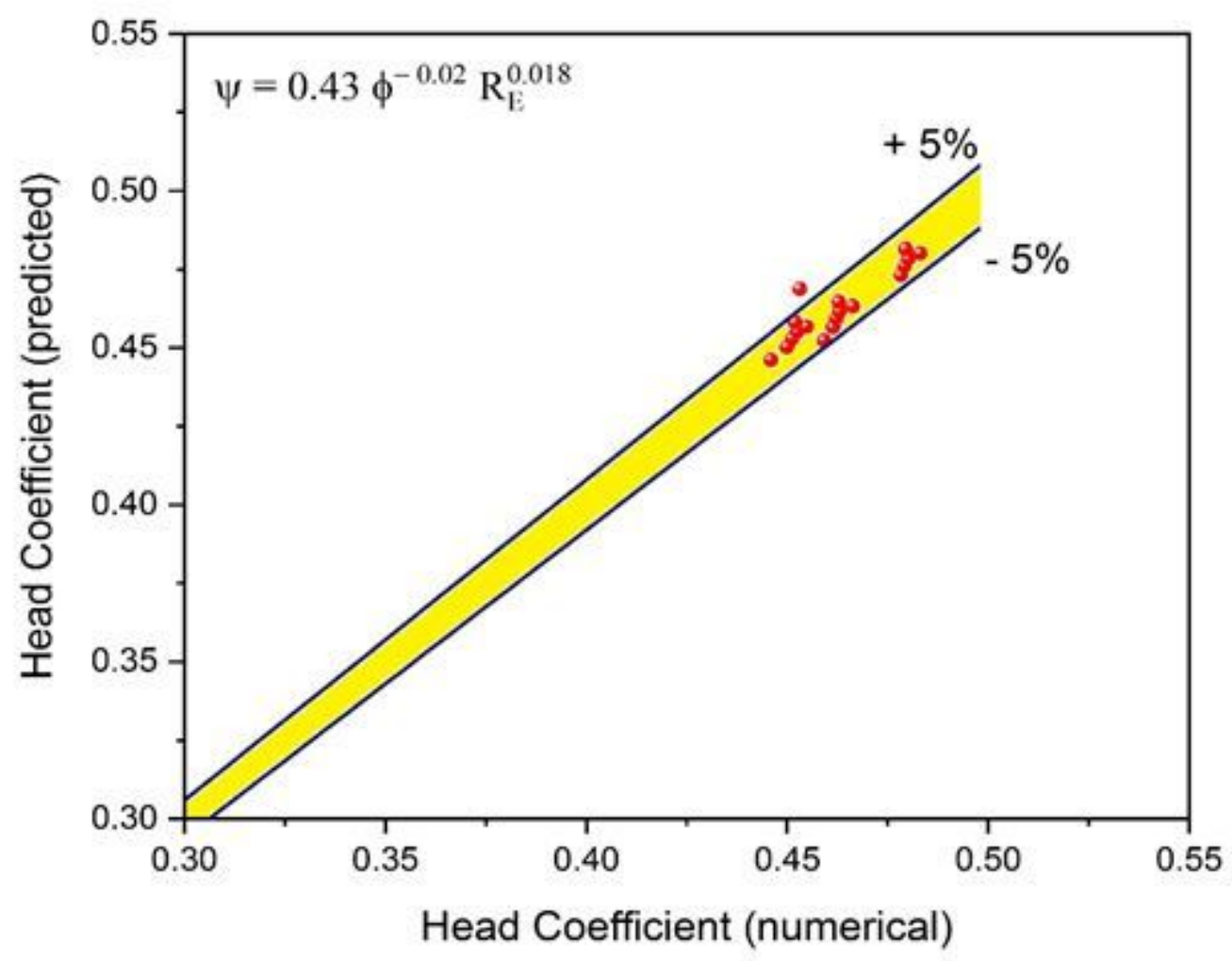

Figure 28

Comparison of numerical and predicted values of Head Coefficient for ellipsoidal hub geometry configuration

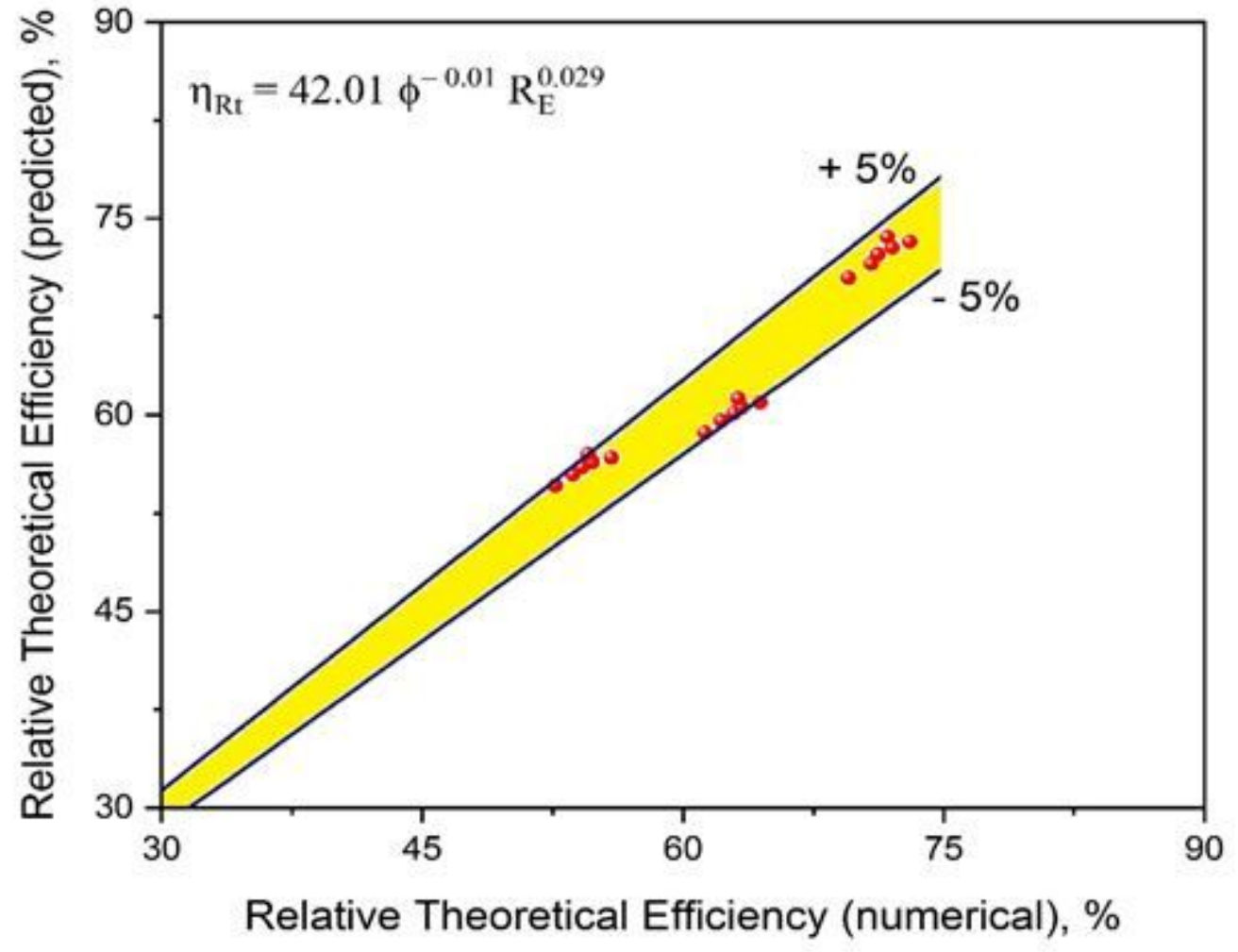

Figure 29 
Comparison of numerical and predicted values of Relative Theoretical Efficiency for ellipsoidal hub geometry configuration 